Supporting Information for

\title{
UV Action Spectroscopy of Gas-Phase Peptide Radicals
}

\author{
Huong T. H. Nguyen, Christopher J. Shaffer, Robert Pepin, Frantisek Turecek \\ Department of Chemistry, University of Washington, Seattle, WA 981195-1700, USA
}

Manuscript submitted to J. Phys. Chem. Lett.

Contents:

1. Experimental Details

2. Computations

3. 3. Supplemental References

Tables 1-3 with $\boldsymbol{z}_{4}(\mathrm{AWAR}), \boldsymbol{z}_{\mathbf{4}}(\mathrm{AFAR})$ and $[\mathrm{AAWAR}+2 \mathrm{H}]^{2+}$ ion relative energies.

Figures S1-S13.

Tables S4-S14 with M06-2X/6-31+G(d,p) fully optimized structures of $z_{4}$ (AWAR) ions.

Tables S15-S22 with M06-2X/6-31+G(d,p) fully optimized structures of $\boldsymbol{z}_{4}$ (AFAR) ions.

Tables S23-S28 with M06-2X/6-31+G(d,p) fully optimized structures of $[\mathrm{AAWAR}+2 \mathrm{H}]^{2+}$ ions.

\section{Experimental Details}

Materials. All peptides were synthesized on Wang resin (Bachem Americas, Torrance, CA, USA) using commercially available Fmoc peptides (Life Technologies, Rockford, IL, USA) according to previously described procedures. ${ }^{\mathbf{s 1}, \mathbf{s 2}}$ Ion formation by electrospray ionization and eletron transfer dissociation to produce $\mathrm{z}$ ions was carried out under standard conditions, as reported previously. ${ }^{\mathbf{3} 3}$ The ETD mass spectra of several AAXAR dications have been reported previously. ${ }^{4 . s 5}$ Typically, fifty scans were accumulated in each spectrum.

Photodissociation. To accomplish photodissociation of trapped ions in the LIT, the LTQ-XL ETD mass spectrometer was modified as reported previously. ${ }^{\mathbf{s 6}, \mathbf{s 7}}$ The irradiating light beam was produced by an Nd-YAG EKSPLA NL301G laser (Altos Photonics, Bozeman, MT, USA) operating at $20 \mathrm{~Hz}$ frequency with a 3-6 ns pulse width. Photons exiting the pump laser are fed into a PG142C unit (Altos Photonics, Bozeman, MT, USA which integrates a third harmonic generator and optical parametric oscillator coupled with an optional second harmonic generator (SH) to provide wavelength tuning between $210-409 \mathrm{~nm}$ at a pulse peak power ranging between 0.79-2.06 mJ. These powers are measured at each wavelength using an EnergyMax-USB J10MB energy sensor (Coherent Inc., Santa Clara, CA, USA) to which the relative laser pulse power is later calibrated as the wavelength is changed. The laser beam is aligned by mirrors and focused by a telescopic lens to pass the small aperture drilled in the auxiliary chemical ionization source source used to produce electron donor reagent ions. The laser beam diameter in the LIT is estimated at 3-4 $\mathrm{mm}$ to ensure overlap with the trapped ions. The beam position does change by about $3 \mathrm{mrad}$ in switching between the SSH/FSH region (210-354 nm) and the ESH region (355$409 \mathrm{~nm}$ ) which requires the realignment of mirrors. This realignment does inevitably change the cross section of the ion cloud exposed to irradiation, and different ratios of dissociation are observed in comparing the regions at normalized power and pulse frequency. The typical number of pulses was 19 for the ESH (355-409 nm) region and 4 for the SSH/FSH (210-354 nm) region. 
Thus, the reported relative fragment ion intensities at each laser wavelength $\left[I_{\text {ion }}(\lambda)\right]$ have been scaled to compensate for this change according to the formula: $\left[I_{\text {ion }}(\lambda)\right]_{\text {scaled }}=$ $\left\{\left[I_{\text {ion }}(\lambda)\right] / \Sigma\left[I_{\text {ion }}(\lambda)\right]\right\} \times\{1 / \mathrm{P}(\lambda)\}$, where $\mathrm{P}(\lambda)$ is the laser power for the given number of pulses. The typical experimental set up consists of selecting the ion to be photodissociated and storing it in the LIT for a chosen time period. For example, 400-ms storage time can accommodate up to 7 laser pulses spaced by $50 \mathrm{~ms}$. This allows one to vary the number of pulses which are also normalized as the number of pulses used is varied depending on the degree of dissociation, such that the ratio of fragmentation products to parent ion does not become 'saturated'. Power dependence measurements of photodissociation efficiency at 358 and $264 \mathrm{~nm}$ showed an approximately linear decrease of the precursor ion relative intensity with laser power, indicating single photon excitation.

\section{Computations}

Standard ab initio and density functional theory calculations were performed with the Gaussian 09 suite of programs. ${ }^{\mathbf{s 8}}$ All structures were obtained by gradient optimization using the B3LYP ${ }^{\mathbf{s} 9}$ and M06-2X ${ }^{\text {s10 }}$ hybrid functionals with the $6-31+\mathrm{G}(\mathrm{d}, \mathrm{p})$ basis set and confirmed as local energy minima by harmonic frequency calculations which gave all real frequencies. The optimized structures are given in Tables S4-S28. All calculations of open-shell species were performed for doublet spin states within the spin-unrestricted formalism. Vertical excitation energies for the doublet spin states were obtained by time-dependent DFT calculations ${ }^{\text {s11 }}$ using the B3LYP, M06-2X, LC-BLYP ${ }^{s 12}$ and $\omega B$ 97X-D ${ }^{\text {s13 }}$ functionals and the $6-311++\mathrm{G}(2 \mathrm{~d}, \mathrm{p})$ basis set in a spin unrestricted formalism. Typically 20-25 excited states were generated by TD-DFT calculations and those with spin-expectation values $<1.5$ were considered. Single-point energies were obtained by calculations that used the above DFT methods and Møller-Plesset perturbational treatment ${ }^{\mathrm{s} 14}$ (MP2, frozen core) with the 6-311++G(2d,p) basis set.

\section{Supplemental References}

(s1) Janz, J. M. ; Ren, Y.; Looby, R.; Kazmi, M. A.; Sachdev, P.; Grunbeck, A.; Haggis, L.; Chinnapen, D.; Lin, A. Y.; Seibert, C.; McMurry, T.; Carlson, K. E.; Muir, T. W.; Hunt, S.; Sakmar, T. P. Direct Interaction between an Allosteric Agonist Pepducin and the Chemokine Receptor CXCR4. J. Am. Chem. Soc. 2011, 133, 15878-15881.

(s2) Coste, J.; Le-Nguyen, D.; Castro, B. PyBOP®: A New Peptide Coupling Reagent Devoid of Toxic By-Product. Tetrahedron Lett. 1990, 31, 205-208.

(s3) Nguyen, H. T. H.; Shaffer, C. J.; Tureček, F. Probing Peptide Cation-Radicals by Near-UV Photodissociation in the Gas Phase. Structure Elucidation of Histidine Radical Chromophores Formed by Electron Transfer Reduction. J. Phys. Chem. B 2015, 119, 3948-3961.

(s4) Chung, T. W.; Hui, R.; Ledvina, A. R.; Coon, J. J.; Tureček, F. Cascade Dissociations of Peptide Cation-Radicals. Part 1. Scope and Effects of Amino Acid Residues in Penta-, Nonaand Decapeptides. J. Am. Soc. Mass Spectrom. 2012, 23, 1336-1350.

(s5) Tureček, F.; Moss, C. L.; Chung, T. W. Correlating ETD Fragment Ion Intensities with Peptide Ion Conformational and Electronic Structure. Int. J. Mass Spectrom. 2012, 330-332, $207-$ 219.

(s6) Shaffer, C. J.; Marek, A.; Pepin, R.; Slováková, K.; Tureček, F. Combining UV

Photodissociation with Electron Transfer for Peptide Structure Analysis J. Mass Spectrom. 2015, $50,470-475$. 
(s8) Frisch, M. J.; Trucks, G. W.; Schlegel, H. B.; Scuseria, G. E.; Robb, M. A.; Cheeseman, J. R.; Scalmani, G.; Barone, V.; Mennucci, B.; Petersson, G. A.; Nakatsuji, H.; Caricato, M.; Li, X.; Hratchian, H. P.; Izmaylov, A. F.; Bloino, J.; Zheng, G.; Sonnenberg, J. L.; Hada, M.; Ehara, M.; Toyota, K.; Fukuda, R.; Hasegawa, J.; Ishida, M.; Nakajima, T.; Honda, Y.; Kitao, O.; Nakai, H.; Vreven, T.; Montgomery, J. A., Jr.; Peralta, J. E.; Ogliaro, F.; Bearpark, M.; Heyd, J. J.; Brothers, E.; Kudin, K. N.; Staroverov, V. N.; Kobayashi, R.; Normand, J.; Raghavachari, K.; Rendell, A.; Burant, J. C.; Iyengar, S. S.; Tomasi, J.; Cossi, M.; Rega, N.; Millam, J. M.; Klene, M.; Knox, J. E.; Cross, J. B.; Bakken, V.; Adamo, C.; Jaramillo, J.; Gomperts, R.; Stratmann, R. E.; Yazyev, O.; Austin, A. J.; Cammi, R.; Pomelli, C.; Ochterski, J. W.; Martin, R. L.; Morokuma, K.; Zakrzewski, V. G.; Voth, G. A.; Salvador, P.; Dannenberg, J. J.; Dapprich, S.; Daniels, A. D.; Farkas, O.; Foresman, J. B.; Ortiz, J. V.; Cioslowski, J.; Fox, D. J.: Gaussian 09, Revision A.02, Gaussian, Inc., Wallingford CT, 2009.

(s9) Becke, A. D. A New Mixing of Hartree-Fock and Local Density-Functional Theories. $J$. Chem. Phys. 1993, 98, 1372-1377.

(s10) Zhao, Y.; D. G. Truhlar, D. G. The M06 Suite of Density Functionals for Main Group Thermochemistry, Thermochemical Kinetics, Noncovalent Interactions, Excited States, and Transition Elements: Two New Functionals and Systematic Testing of Four M06-Class Functionals and 12 Other Functionals. Theor. Chem. Acc. 2008, 120, 215-241.

(s11) Furche, F.; Ahlrichs, A. Adiabatic Time-Dependent Density Functional Methods for Excited State Properties. J. Chem. Phys. 2002, 117, 7433-7447.

(s12) Iikura, H.; Tsuneda, T.; Yanai, T.; Hirao, K. A Long-Range Correction Scheme for Generalized-Gradient-Approximation Exchange Functionals. J. Chem. Phys. 2001, 115, 35403544.

(s13) Chai, J. D.; Head-Gordon, M. Long-Range Corrected Hybrid Density Functionals with Damped Atom-Atom Dispersion Corrections. Phys. Chem. Chem. Phys. 2008, 10, 6615-6620.

(s14) Møller, C.; Plesset, M. S. A Note on an Approximation Treatment for Many-Electron Systems. Phys. Rev. 1934, 46, 618-622. 
Table S1. Relative Energies of $\boldsymbol{z}_{\mathbf{4}}\left(\mathrm{AWAR}^{+}\right)$Isomers.

$$
\text { Relative Energy }{ }^{a, b}
$$

\begin{tabular}{|c|c|c|c|c|c|}
\hline Ion & $\mathrm{B} 3 \mathrm{LYP}^{c}$ & M06-2X $\mathrm{X}^{d}$ & $\omega \mathrm{B} 97 \mathrm{X}-\mathrm{D}^{c}$ & LC-BLYP ${ }^{c}$ & $\mathrm{ROMP}^{c}$ \\
\hline $1 \mathbf{a}$ & 0.0 & 0.0 & 0.0 & 0.0 & 0.0 \\
\hline $1 b$ & 2.9 & 26 & 14 & 15 & - \\
\hline $1 c$ & -1.1 & 34 & 8.3 & 11 & - \\
\hline $1 d$ & 0.1 & 17 & -1.2 & 5.7 & 15 \\
\hline $2 \mathbf{a}$ & -31 & 3.6 & -13 & -17 & - \\
\hline $2 b$ & -16 & -10 & -4 & -4.8 & - \\
\hline $\mathbf{3 a}$ & -37 & -20 & -25 & -28 & - \\
\hline $\mathbf{3 b}$ & -34 & -2.3 & -12 & -19 & - \\
\hline $4 a$ & -43 & -38 & -43 & -40 & - \\
\hline $4 b$ & -11 & $-38^{x}$ & 4 & & \\
\hline $5 \mathbf{a}$ & -21 & 8.3 & -0.1 & -11 & 4.7 \\
\hline $5 b$ & -6 & 36 & 20 & 10 & - \\
\hline
\end{tabular}

${ }^{a}$ In units of $\mathrm{kJ} \mathrm{mol}^{-1}$. ${ }^{b}$ Including zero point energy corrections. ${ }^{c}$ From single-point energy calculations with the 6-311++G(2d,p) basis set on B3LYP/6-31+G(d,p) optimized geometries. ${ }^{d}$ From single-point energy calculations with the $6-311++\mathrm{G}(2 \mathrm{~d}, \mathrm{p})$ basis set on M06-2X/6$31+\mathrm{G}(\mathrm{d}, \mathrm{p})$ optimized geometries. 
Table S2. Relative Energies of $\boldsymbol{z}_{\mathbf{4}}\left(\mathrm{AFAR}^{+}\right)$Isomers.

$$
\text { Relative Energy }{ }^{a, b}
$$

\begin{tabular}{|c|c|c|c|c|c|}
\hline Ion & $\mathrm{B} 3 \mathrm{LYP}^{c}$ & M06-2X ${ }^{d}$ & $\omega \mathrm{B} 97 \mathrm{X}-\mathrm{D}^{c}$ & $\mathrm{LC}^{-B L Y P}{ }^{c}$ & $\mathrm{ROMP}^{c}$ \\
\hline $6 \mathbf{a}$ & 0.0 & 0.0 & 0.0 & 0.0 & $0.0(0.0)^{e}$ \\
\hline $6 \mathbf{b}$ & 5.8 & $-x$ & 5.1 & 14 & $19(19)$ \\
\hline 7 & -8.4 & 1.1 & -2.9 & -1.5 & $-3.9(-5.2)$ \\
\hline $8 \mathbf{a}$ & -34 & -14 & -21 & -22 & $-16(-14)$ \\
\hline $8 b$ & -0.6 & 14 & 10 & 14 & - \\
\hline $9 \mathbf{a}$ & -25 & -56 & -49 & -34 & $-54(-52)$ \\
\hline $9 \mathrm{~b}$ & -32 & -55 & -44 & -37 & $-46(-45)$ \\
\hline $10 \mathbf{a}$ & -20 & -4.0 & -8.5 & -16 & $-6.1(-4.9)$ \\
\hline $10 b$ & -12 & 28 & 2.8 & -3.0 & $9.8(11)$ \\
\hline
\end{tabular}

${ }^{a}$ In units of $\mathrm{kJ} \mathrm{mol}^{-1} .{ }^{b}$ Including zero point energy corrections and referring to $0 \mathrm{~K}$. ${ }^{c}$ From singlepoint energy calculations with the $6-311++\mathrm{G}(2 \mathrm{~d}, \mathrm{p})$ basis set on B3LYP/6-31+G(d,p) optimized geometries. ${ }^{d}$ From single-point energy calculations with the $6-311++\mathrm{G}(2 \mathrm{~d}, \mathrm{p})$ basis set on M062X/6-31+G(d,p) optimized geometries. ${ }^{e}$ Spin-projected PMP2/6-311++G(2d,p) energies. ${ }^{x}$ M06$2 \mathrm{X} / 6-31+\mathrm{G}(\mathrm{d}, \mathrm{p})$ geometry optimizations converged to a single structure $(\mathbf{6 a})$. 
Table S3. Relative Energies of $(A A W A R+2 H)^{2+}$ Conformers. See Figure S7 for optimized structures.

\begin{tabular}{|c|c|c|c|c|c|c|}
\hline \multirow[b]{2}{*}{ Ion } & \multirow[b]{2}{*}{ Type } & \multicolumn{5}{|c|}{ Relative Energy ${ }^{a, b}$} \\
\hline & & $\mathrm{B}^{2} \mathrm{LYP}^{c}$ & M06- $2 \mathrm{X}^{d}$ & $\omega \mathrm{B} 97 \mathrm{X}-\mathrm{D}^{c}$ & LC-BLYP ${ }^{c}$ & $\mathrm{MP}^{\circ}$ \\
\hline $\mathbf{A}$ & non-stacked & 0.0 & 0.0 & 0.0 & 0.0 & 0.0 \\
\hline B & non-stacked & 1.5 & 14 & 2.2 & 3.8 & 2.8 \\
\hline C & $\operatorname{Arg}^{+} \ldots \operatorname{Trp}$ & 16 & 24 & 14 & 11 & 13 \\
\hline D & $\operatorname{Arg}^{+} \ldots \operatorname{Trp}$ & 15 & 22 & 12 & 13 & 12 \\
\hline $\mathbf{E}$ & $\mathrm{Lys}^{+} \ldots$ Trp & -14 & 10 & -5.1 & -0.8 & 4.8 \\
\hline $\mathbf{F}$ & Lys $^{+} \ldots$ Trp & -4.0 & 20 & 4.3 & 12 & 15 \\
\hline
\end{tabular}

${ }^{a}$ Relative free energies in $\mathrm{kJ} \mathrm{mol}^{-1} .{ }^{b}$ Including zero-point corrections and $298 \mathrm{~K}$ enthalpies and entropies. ${ }^{c}$ Single-point calculations with the $6-311++\mathrm{G}(2 \mathrm{~d}, \mathrm{p})$ basis set on B3LYP/6-31+G(d,p) optimized geometries. ${ }^{d}$ Single-point calculations with the $6-311++\mathrm{G}(2 \mathrm{~d}, \mathrm{p})$ basis set on M06$2 \mathrm{X} / 6-31+\mathrm{G}(\mathrm{d}, \mathrm{p})$ optimized geometries. 

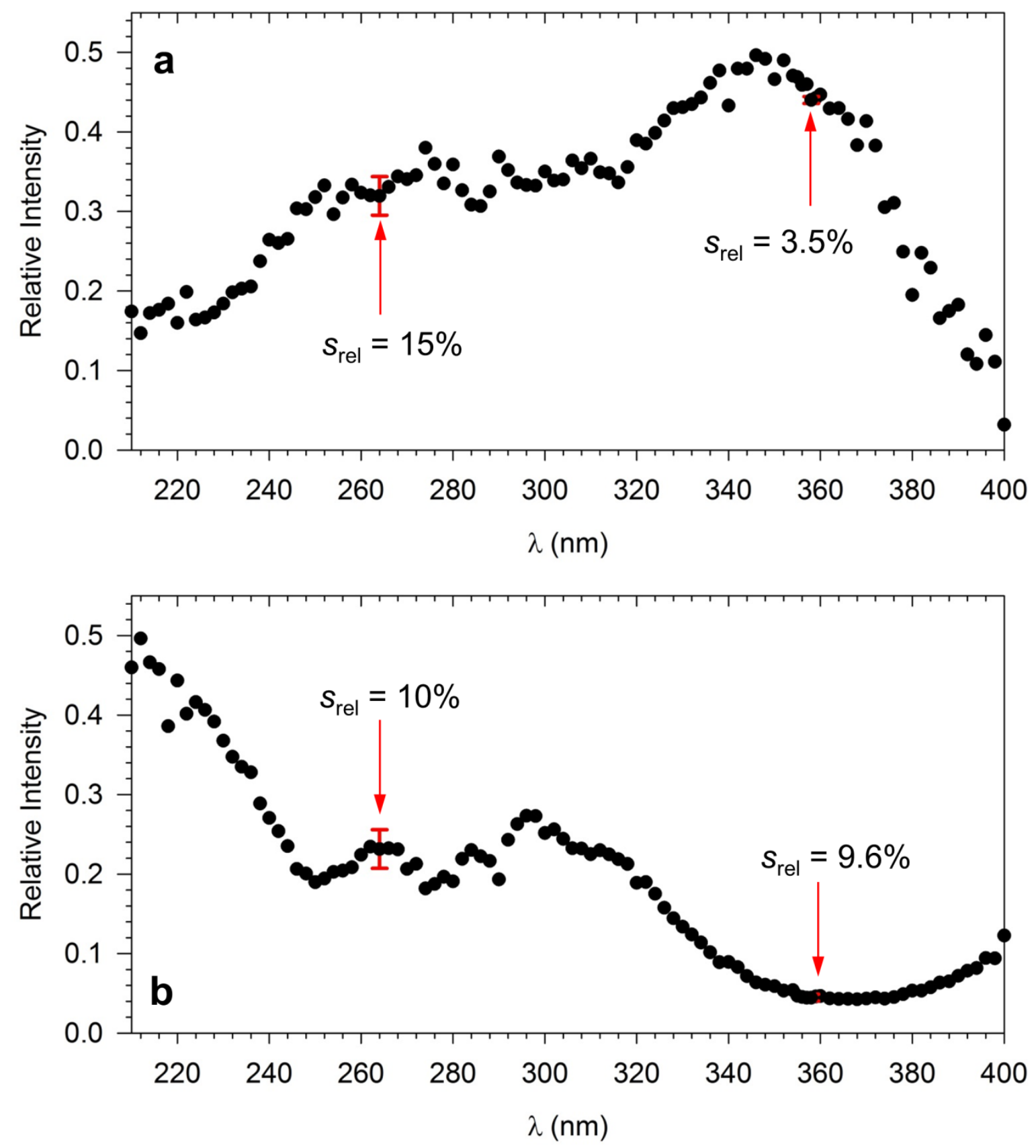

Figure S1. Mass-resolved UV action spectra of $\boldsymbol{z}_{\mathbf{4}}\left({ }^{\bullet} \mathrm{AWAR}^{+}\right)$ions. (a) $\left[\boldsymbol{y}_{\mathbf{2}}-2 \mathrm{H}\right]^{+}(\mathrm{m} / \mathrm{z}, 244)$ and (b) $\left[z_{\mathbf{4}}-\mathrm{H}\right]^{+}(\mathrm{m} / \mathrm{z}, 486)$ channels. The fragment ion intensities are normalized to their sum, the calibrated laser power at each wavelength, and the number of pulses used to obtain the spectrum. The red error bars show relative standard deviations of fragment ion intensities from 10 consecutive measurements at given laser wavelengths. 

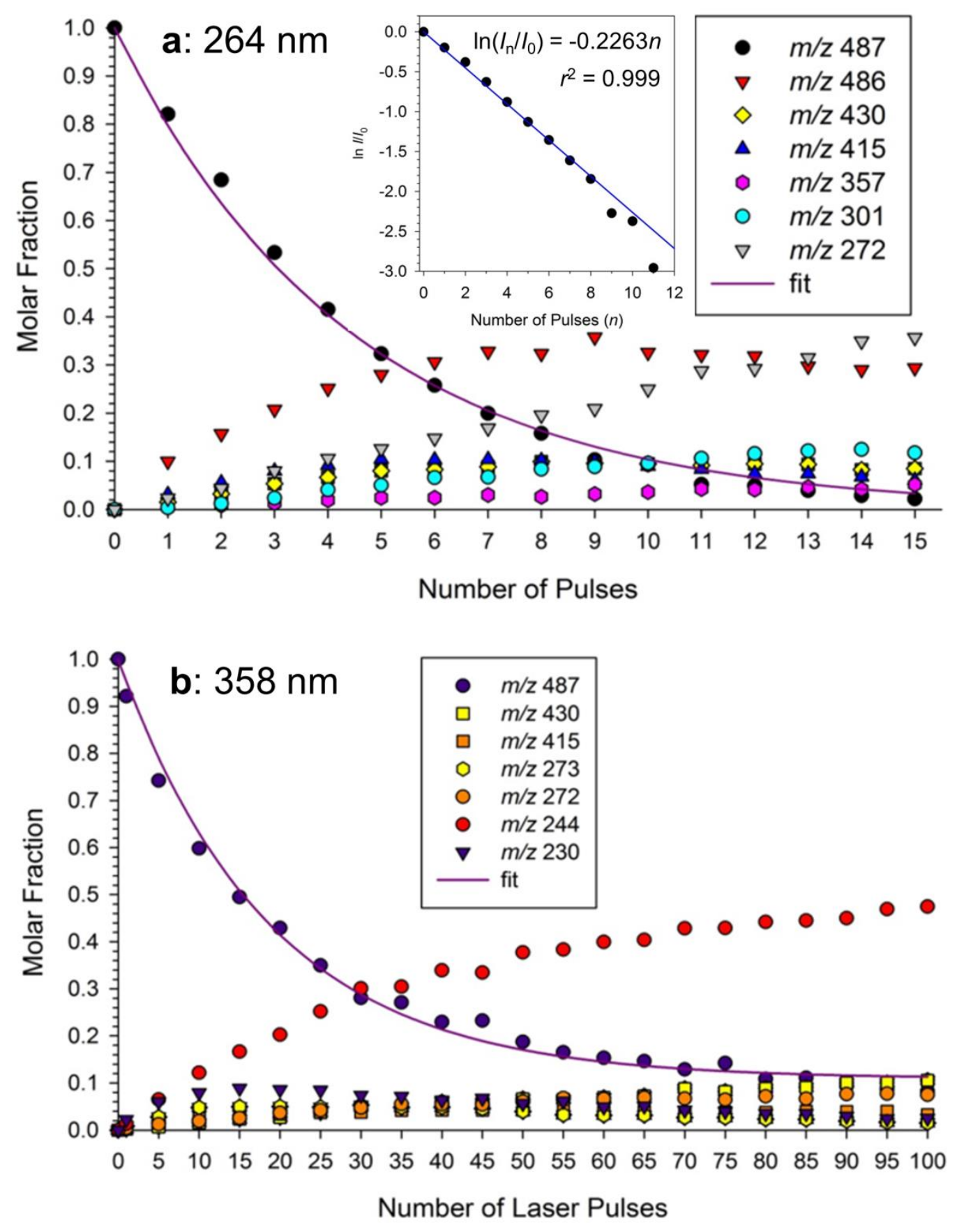

Figure S2. Pulse dependence of $\boldsymbol{z}_{4}\left({ }^{\bullet} \mathrm{AWAR}^{+}\right.$) ion intensities at (a) $264 \mathrm{~nm}$ and (b) $358 \mathrm{~nm}$. Ion assignment is as follows: $\left.\boldsymbol{z}_{\mathbf{4}}{ }^{\bullet}{ }^{\bullet} \mathrm{AWAR}{ }^{+}\right) \mathrm{m} / \mathrm{z}$ 487, $\left[\boldsymbol{z}_{\mathbf{4}}-\mathrm{H}\right]^{+} \mathrm{m} / \mathrm{z}$ 486, $\left[\boldsymbol{y}_{\mathbf{3}}-2 \mathrm{H}\right]^{+} \mathrm{m} / \mathrm{z}, 430, \boldsymbol{x}_{\mathbf{2}}{ }^{+} \mathrm{m} / \mathrm{z}$ $273,\left[\boldsymbol{x}_{\mathbf{2}}-\mathrm{H}\right]^{+} \mathrm{m} / \mathrm{z} 272,\left[\boldsymbol{y}_{\mathbf{2}}-2 \mathrm{H}\right]^{+} \mathrm{m} / \mathrm{z} 244, \boldsymbol{z}_{2}{ }^{+} \mathrm{m} / \mathrm{z} 230$. Inset in panel a shows the ln plot of $\mathrm{m} / \mathrm{z}$ 487 relative intensity. 


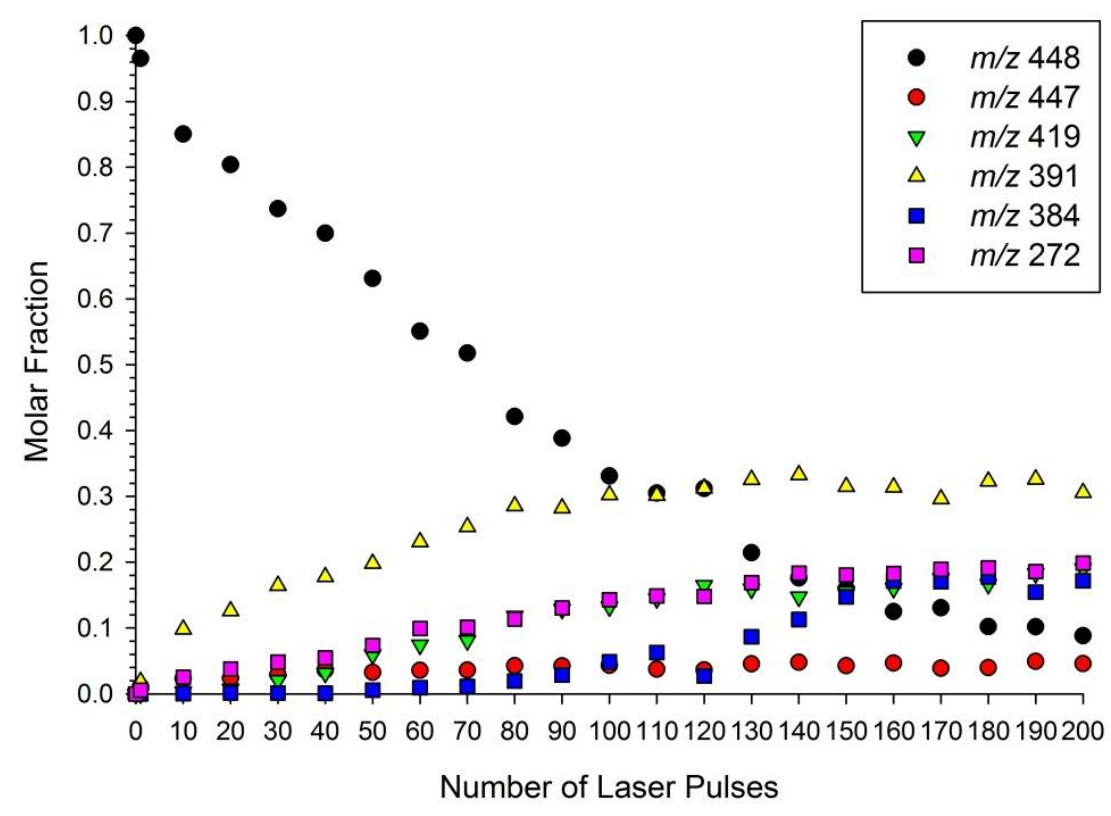

Figure S3. Pulse dependence of the $z_{4}\left({ }^{\bullet} \mathrm{AFAR}^{+}\right)$and photofragment ion intensities at $275 \mathrm{~nm}$. Black circles: $z_{\mathbf{4}}\left({ }^{\bullet} \mathrm{AFAR}^{+}\right)\left(\mathrm{m} / z\right.$ 448); red circles: $\left[z_{\mathbf{4}}-\mathrm{H}\right]^{+}\left(\mathrm{m} / z\right.$ 447); green triangles: $\left[z_{\mathbf{4}}-29\right]^{+}$ $\left(\mathrm{m} / \mathrm{z}\right.$ 419); yellow triangles: $\left[\boldsymbol{y}_{\mathbf{3}}-2 \mathrm{H}\right]^{+}(\mathrm{m} / \mathrm{z} 391)$; blue squares: unassigned $(\mathrm{m} / \mathrm{z} 384)$; pink squares: $\left[\boldsymbol{x}_{\mathbf{2}}-\mathrm{H}\right]^{+}(\mathrm{m} / \mathrm{z} 272)$.

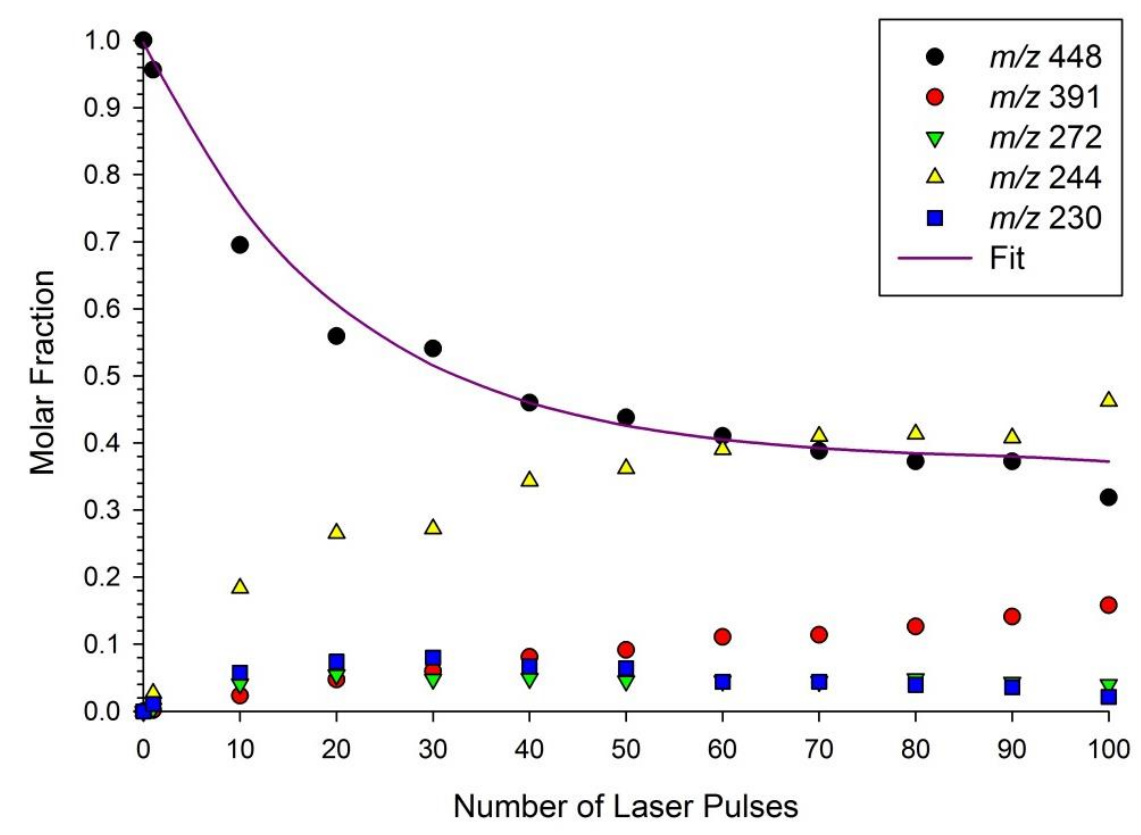

Figure S4. Pulse dependence of the $z_{4}\left({ }^{\bullet} \mathrm{AFAR}^{+}\right)$and photofragment ion intensities at $351 \mathrm{~nm}$. Black circles: $\boldsymbol{z}_{\mathbf{4}}\left({ }^{\bullet} \mathrm{AFAR}^{+}\right)(\mathrm{m} / \mathrm{z} 448)$; red circles: $\left[\boldsymbol{y}_{\mathbf{3}}-2 \mathrm{H}\right]^{+}\left(\mathrm{m} / z\right.$ 391); green triangles: $\left[\boldsymbol{x}_{\mathbf{2}}-\mathrm{H}\right]^{+}$ $(\mathrm{m} / \mathrm{z} 272)$; yellow triangles: $\left[\boldsymbol{y}_{\mathbf{2}}-2 \mathrm{H}\right]^{+}(\mathrm{m} / \mathrm{z} 244)$; blue squares: $\boldsymbol{z}_{2}{ }^{+}{ }^{\bullet}(\mathrm{m} / \mathrm{z} 230)$. The fit curve for $m / z, 448$ is $I(n) / I_{0}=0.627 e^{-0.0493 n}+0.373$. 


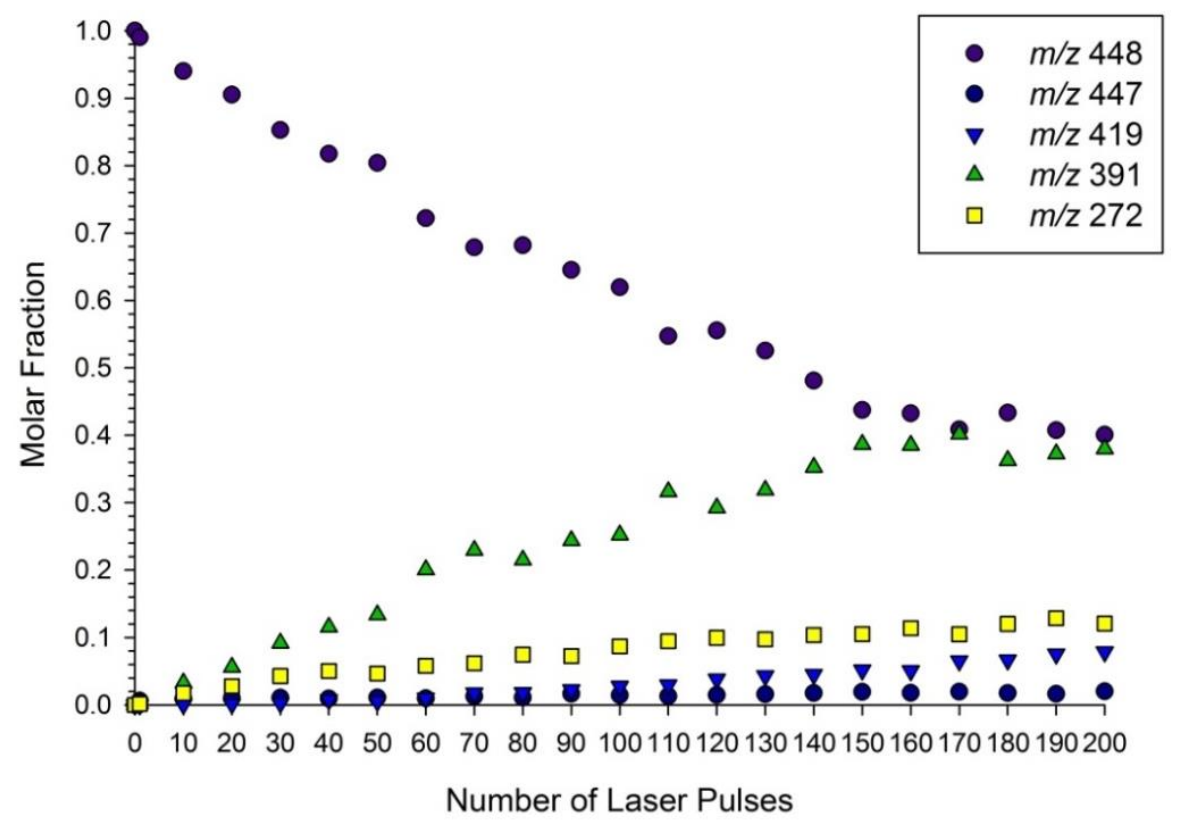

Figure S5. Pulse dependence of the $z_{4}\left({ }^{\bullet} \mathrm{AFAR}^{+}\right)$and photofragment ion intensities at $316 \mathrm{~nm}$. The symbol assignments are as in Figures S1 and S2.

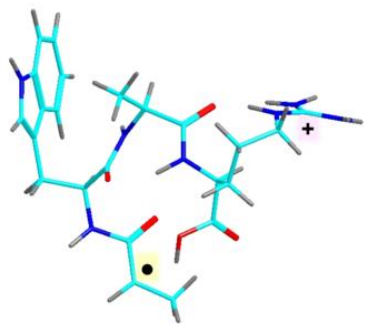

$1 b$
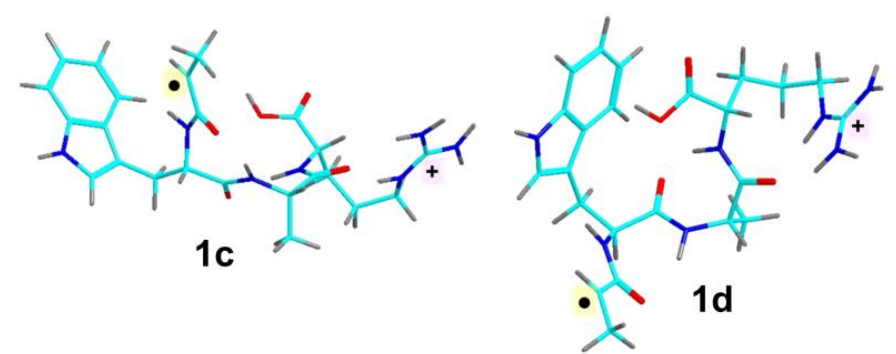

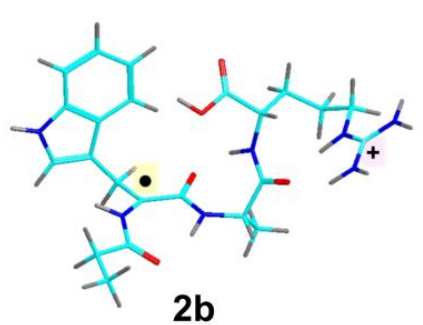

2b
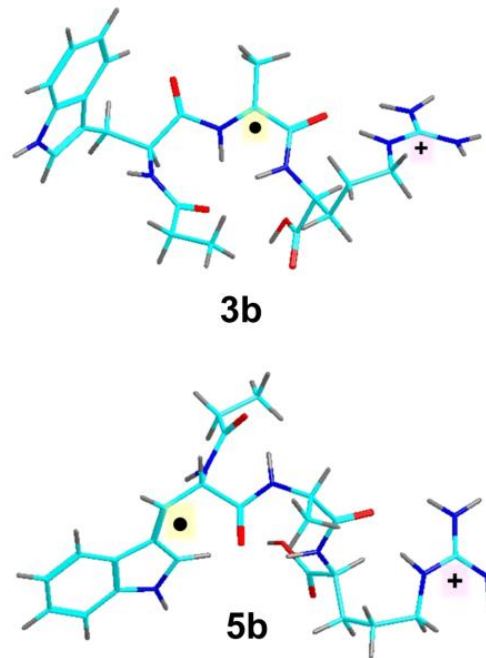

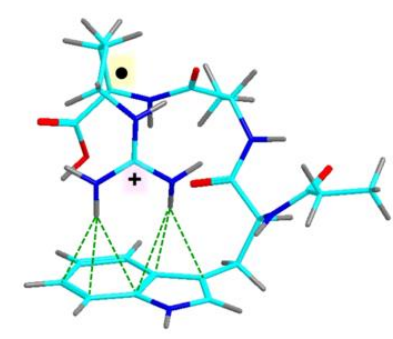

4b

Figure S6. M06-2X/6-31+G(d,p) optimized structures of less-stable conformers of $z_{4}\left({ }^{\bullet} \mathrm{AWAR}^{+}\right)$ isomers. See Table S1 for relative energies. Green dash lines indicate hydrogen bonds to the aromatic ring 

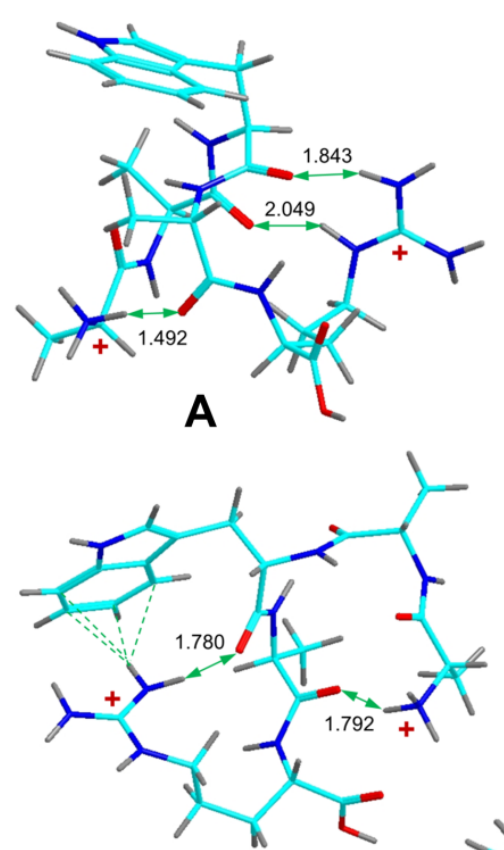

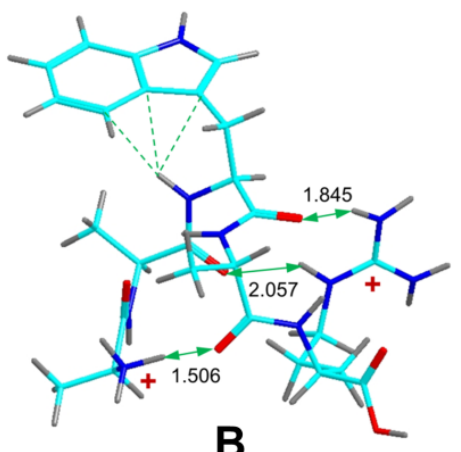

B
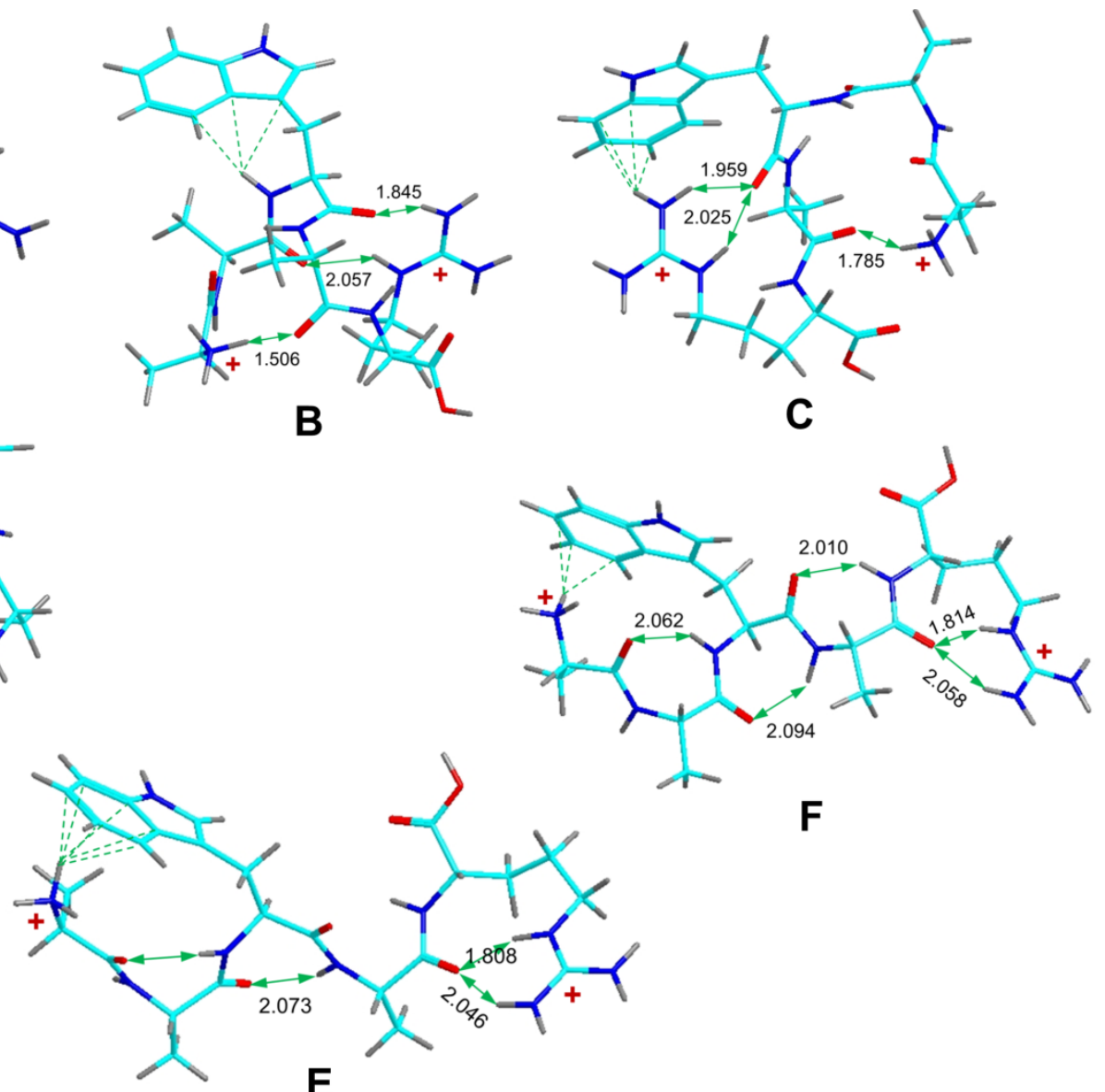

$\mathbf{E}$

Figure S7. M06-2X/6-31+G(d,p) optimized structures of $[\text { AAWAR }+2 \mathrm{H}]^{2+}$ conformers. See Table S3 for relative free energies. Green dash lines and arrows indicate hydrogen bonds with distances given in Ångstrøms. 

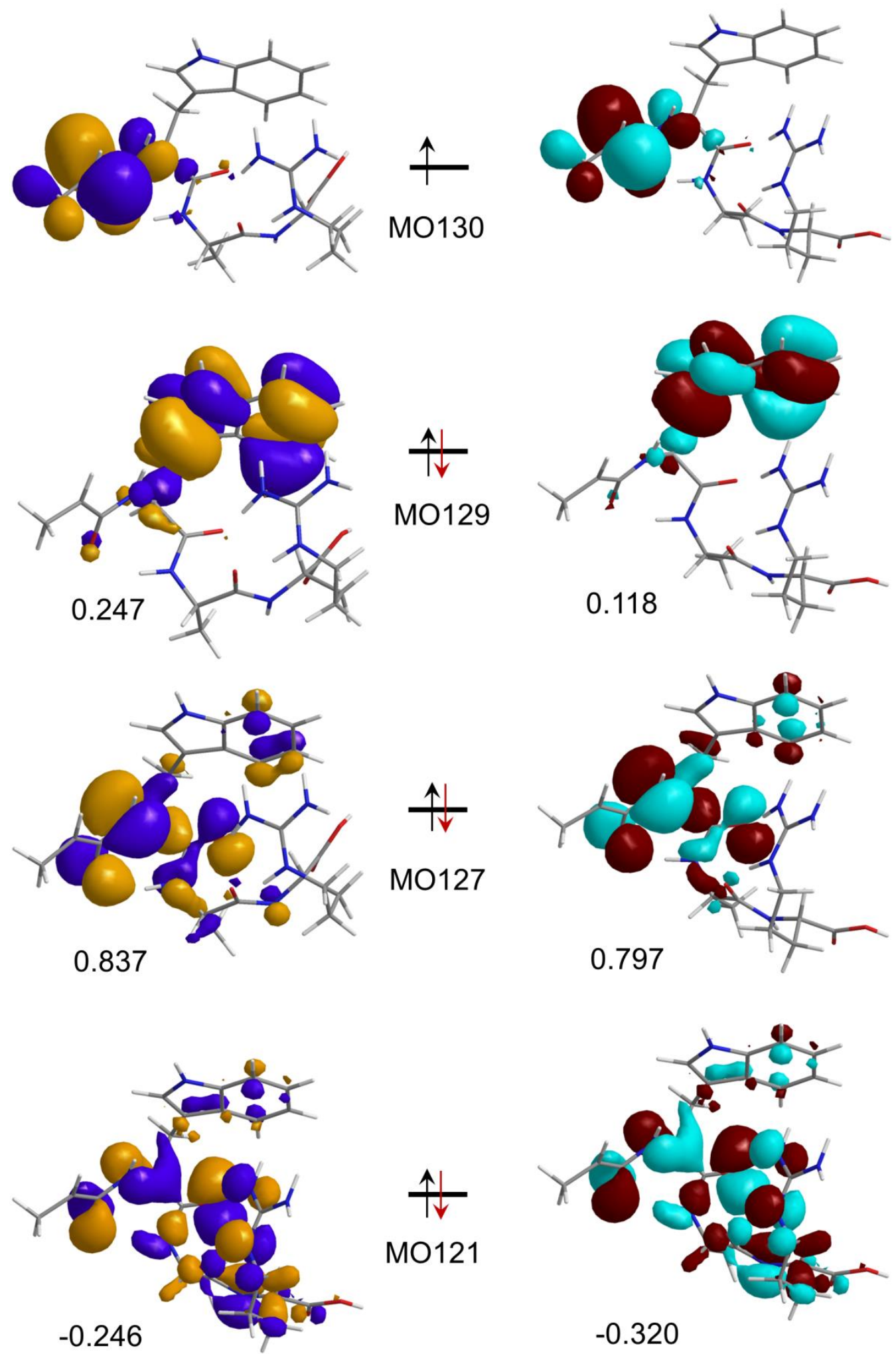

Figure S8. Molecular orbitals for the $\pi \rightarrow \pi$ electron excitation in 1a from TD-DFT calculations. Left panel: M06-2X/6-311++G(2d,p) orbitals and transition vector amplitudes. Right panel: LC-BLYP/6-311++G(2d,p) orbitals and transition vector amplitudes. 

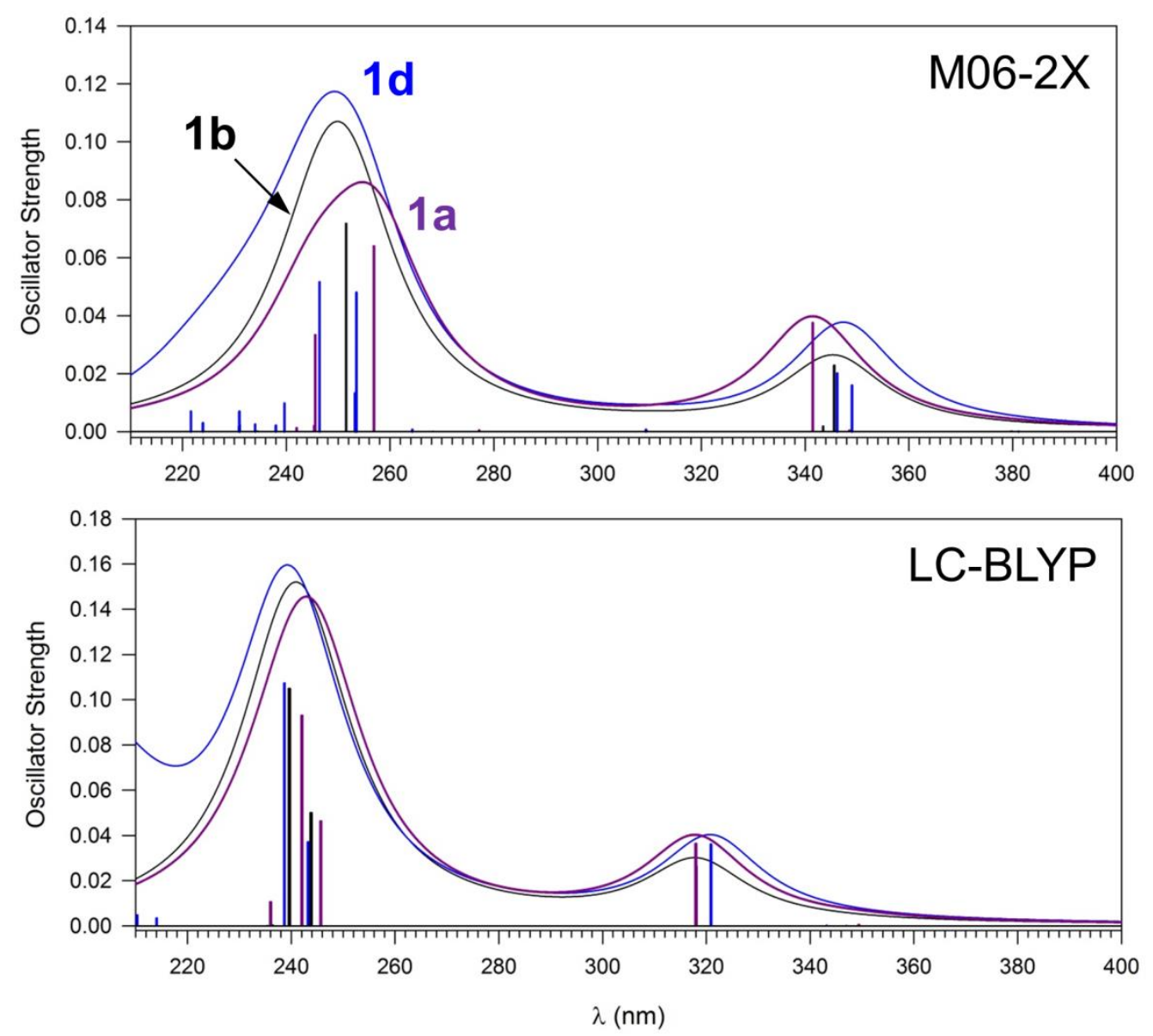

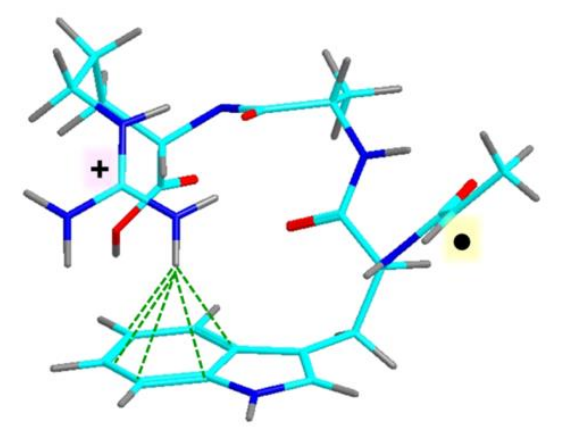

$1 \mathrm{a}$

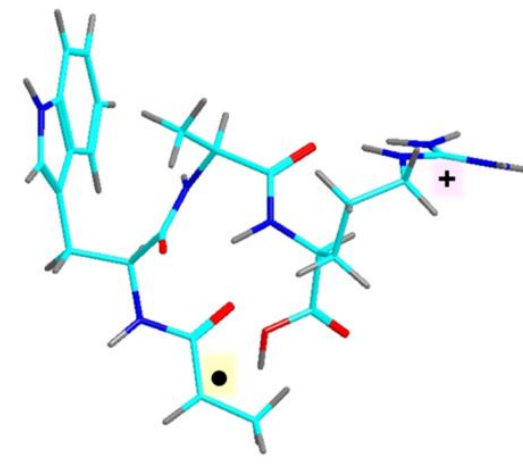

$1 b$

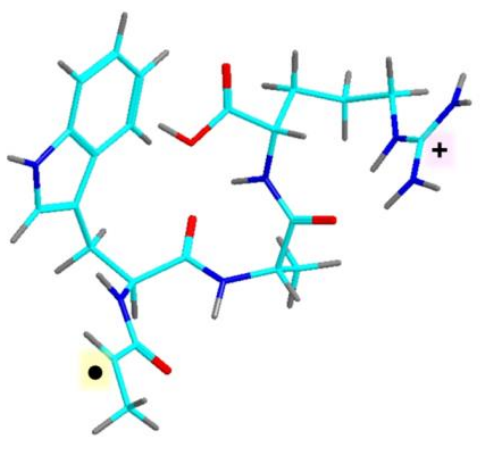

1d

Figure S9. TD-DFT calculated absorption spectra of $z_{4}\left({ }^{\bullet} \mathrm{AWAR}^{+}\right)$conformers $\mathbf{1 a}, \mathbf{1 b}$, and $\mathbf{1 d}$. 

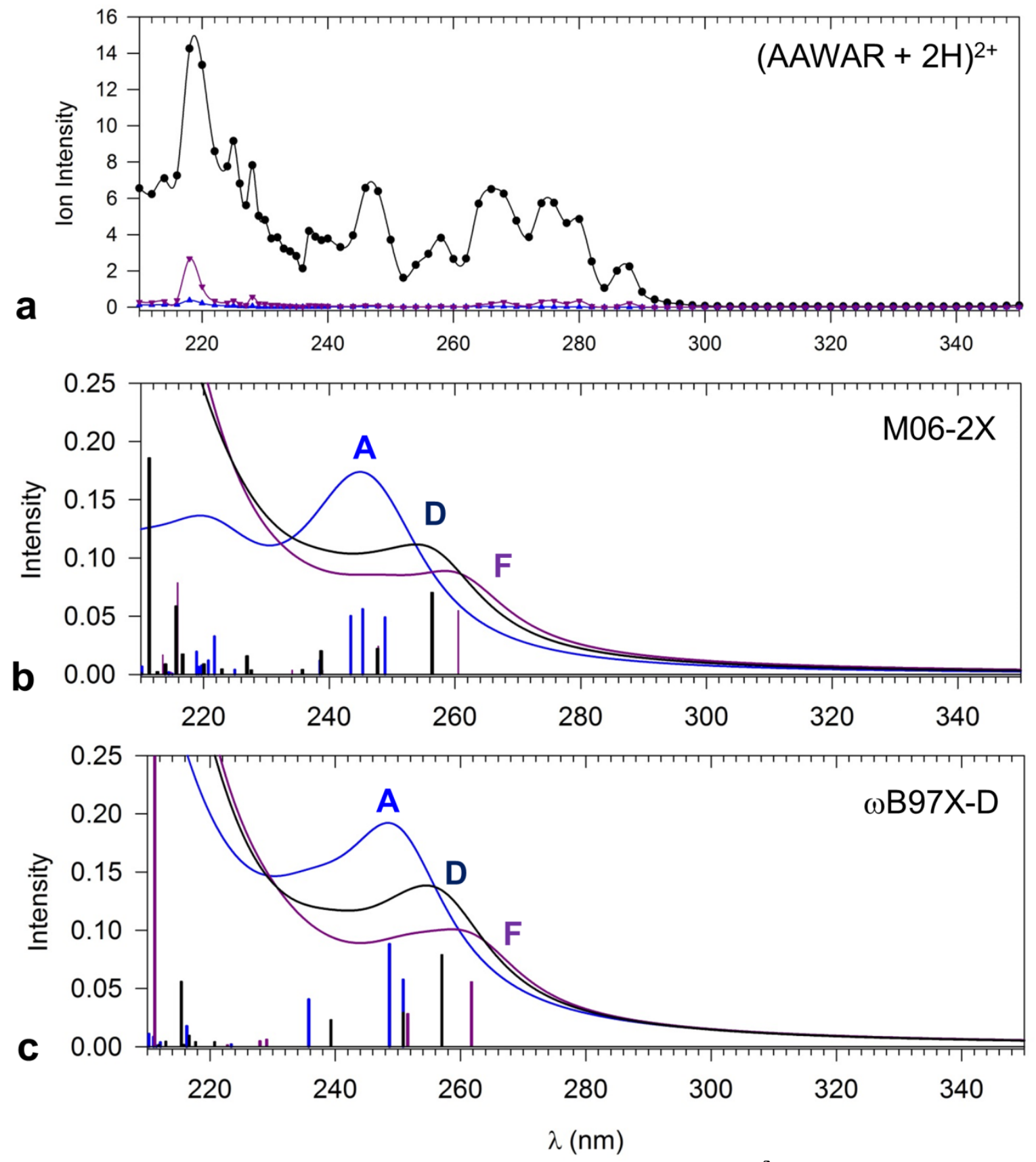

Figure S10. (a) UV Action spectrum of the $[\mathrm{AAWAR}+2 \mathrm{H}]^{2+}$ ion showing wavelengthdependent intensity profiles of the $\boldsymbol{y}_{\mathbf{3}}{ }^{+}\left(\mathrm{m} / \mathrm{z}\right.$ 432, black), $\boldsymbol{a}_{\mathbf{3}}{ }^{+}\left(\mathrm{m} / \mathrm{z} 301\right.$, magenta), and $\boldsymbol{b}_{\mathbf{3}}{ }^{+}(\mathrm{m} / \mathrm{z}, 329$, blue) fragment ions. TD-DFT-calculated absorption spectra of (AAWAR) ${ }^{2+}$ conformers A, D, and F using (b) M06-2X/6-311++G(2d,p) and (c) $\omega \mathrm{B} 97 \mathrm{X}-\mathrm{D} / 6-311++\mathrm{G}(2 \mathrm{~d}, \mathrm{p})$. 

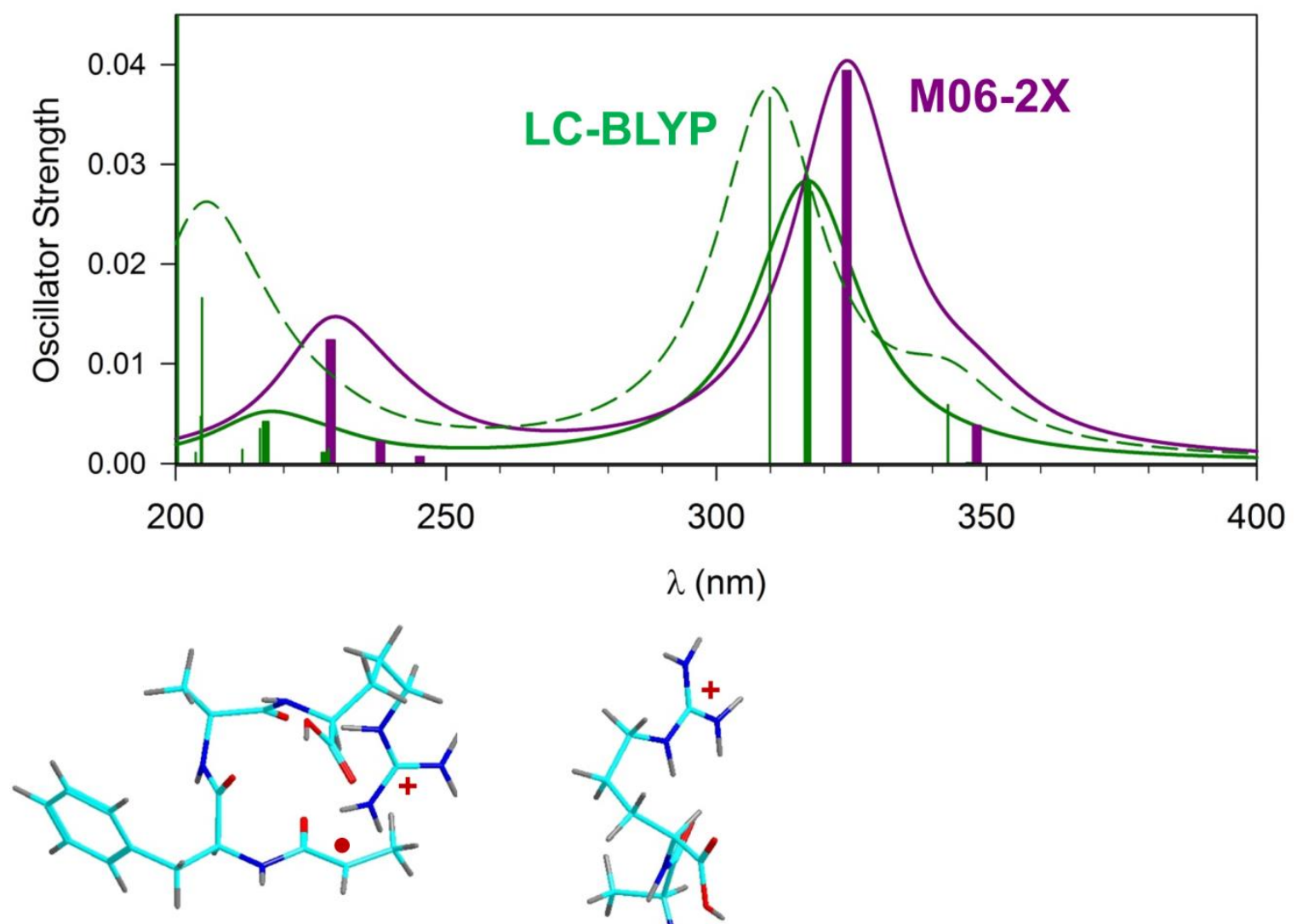

$6 a$

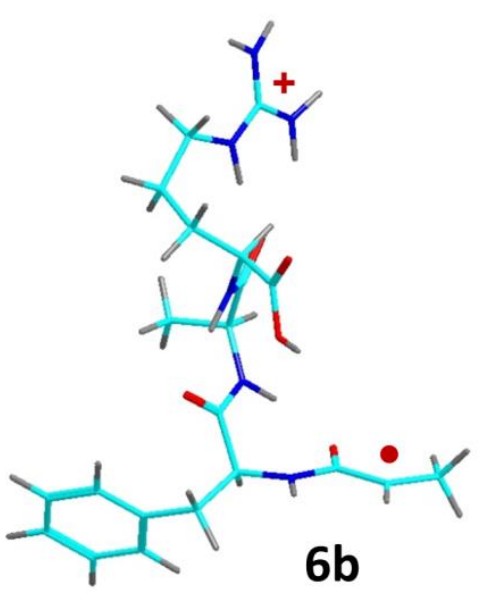

Figure S11. TD-DFT-calculated absorption spectra of $\boldsymbol{z}_{4}\left({ }^{\bullet} \mathrm{AFAR}^{+}\right)$conformers $6 \mathbf{a}$ (solid lines) and $\mathbf{6 b}$ (dashed line). 

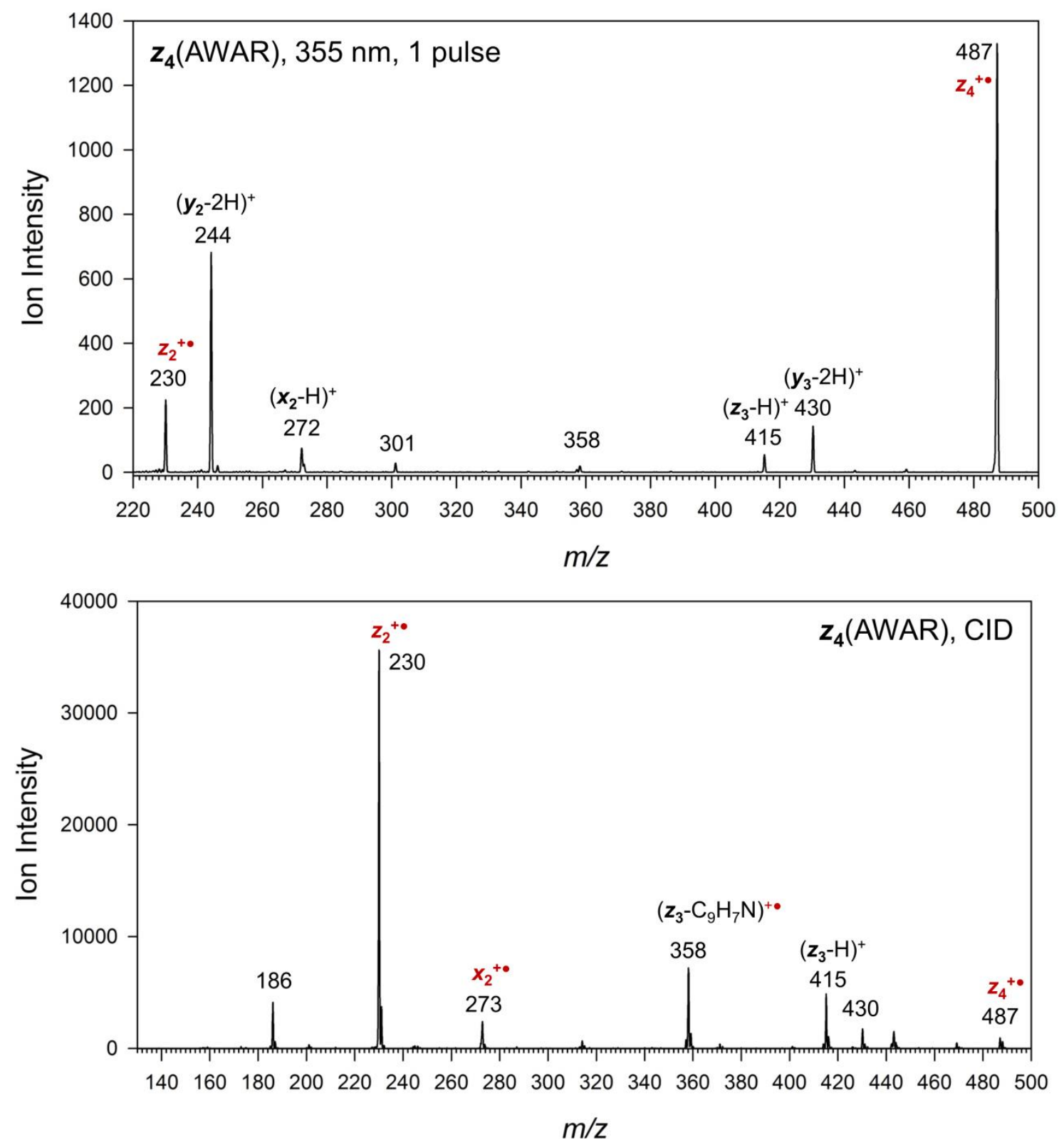

Figure S12. Single-pulse $355 \mathrm{~nm}$ UVPD (top) and CID (bottom) spectra of $z_{4}\left({ }^{\bullet} \mathrm{AWAR}^{+}\right)$ions. 

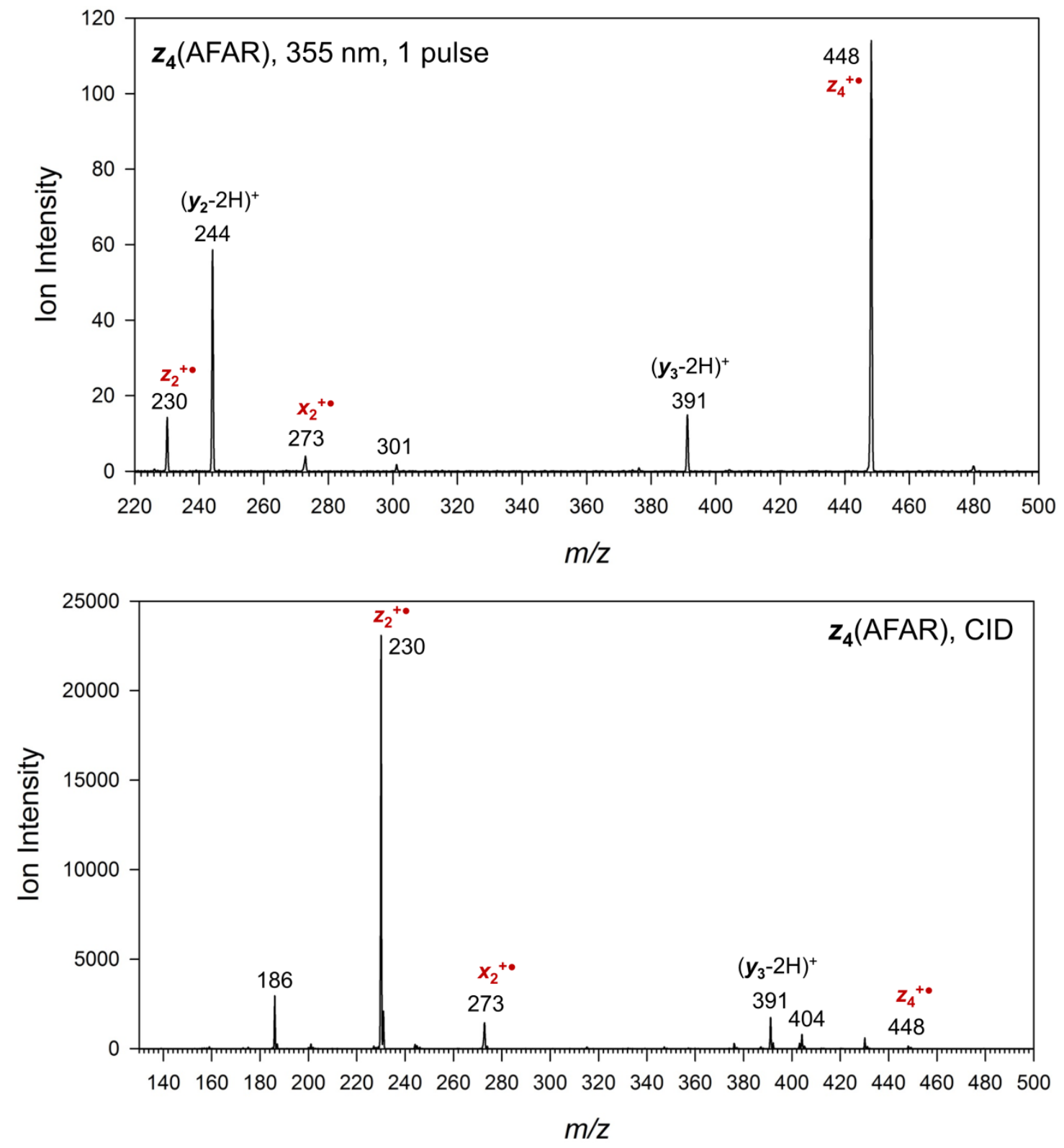

Figure S13. Single-pulse $355 \mathrm{~nm}$ UVPD (top) and CID (bottom) spectra of $z_{4}\left({ }^{\bullet} \mathrm{AFAR}^{+}\right)$ions. 
Table S4. M06-2X/6-31+G(d,p) Optimized Geometry of AWAR Radical 1a.

$\mathrm{C},-4.5865729542,-2.715274717,0.5231848104 \backslash \mathrm{C},-3.7401727943,-2.1$

$965617271,-0.5387537731 \backslash 0,-3.4336715799,-2.8648828461,-1.5359030247 \backslash \mathrm{C}$, $-5.1282081515,-4.0927073922,0.4619110995 \backslash N,-3.2856933537,-0.9124018204$ $,-0.3862422776 \backslash \mathrm{C},-2.5489890719,-0.2172601375,-1.4251645185 \backslash \mathrm{C},-1.050748$ $825,-0.5672416442,-1.3975625077 \backslash 0,-0.1766721226,0.2898970169,-1.264707$ $5371 \backslash \mathrm{C},-2.7951694943,1.3015628184,-1.355456938 \backslash \mathrm{C},-2.7171632009,1.92902$ $93761,0.0099706294 \backslash C,-1.6075096699,2.6094539775,0.6371581223 \backslash C,-2.0677$ $721221,3.0894871368,1.8919224581 \backslash N,-3.3832758731,2.7163505324,2.020821$ $9821 \backslash \mathrm{C},-3.7712397346,2.045210495,0.883683134 \backslash \mathrm{C},-0.2856827709,2.9012965$ $629,0.2442414798 \backslash C, 0.5222238024,3.6367010404,1.0961946648 \backslash C, 0.04342759$ $18,4.0967691664,2.3440611232 \backslash \mathrm{C},-1.2509771359,3.8240359419,2.7649700273$ $\backslash \mathrm{H},-3.9965392114,2.9831817354,2.7751872723 \backslash \mathrm{H},-3.4518474695,-0.41603159$ $94,0.4791408636 \backslash \mathrm{N},-0.7699406581,-1.8785876362,-1.5364096535 \backslash \mathrm{C}, 0.601682$ $4744,-2.3673100104,-1.5811052962 \backslash \mathrm{C}, 1.3873397634,-1.6002122515,-0.50753$ $98476 \backslash 0,0.9750267287,-1.5995607829,0.6564793632 \backslash \mathrm{C}, 1.1759320986,-2.2936$ $776673,-2.9943499053 \backslash \mathrm{H},-1.5473192355,-2.5247016694,-1.6770069764 \backslash \mathrm{N}, 2.5$ $126132447,-0.9577896816,-0.8851483275 \backslash \mathrm{C}, 2.9424073245,0.2483146886,-0.1$ $942484506 \backslash C, 3.0839085977,1.3272698655,-1.2621496715 \backslash 0,3.1334302702,1.1$ $075904964,-2.4455808509 \backslash C, 4.289715015,0.101282043,0.5419803226 \backslash C, 4.326$ $6567536,-0.920691233,1.6831613222 \backslash \mathrm{C}, 3.6669121232,-0.4636690672,2.98784$ $83607 \backslash \mathrm{N}, 2.2107863514,-0.4686702745,2.8553890272 \backslash \mathrm{C}, 1.3978124487,0.57008$ $61356,3.0234361171 \backslash \mathrm{N}, 1.7572898239,1.6247014801,3.7697866509 \backslash \mathrm{N}, 0.198588$ $7037,0.5535692858,2.4393111176 \backslash \mathrm{H}, 1.8314443776,-1.1823842508,2.22721281$ $64 \backslash \mathrm{H}, 0.0531833423,-0.0742322068,1.6503546276 \backslash \mathrm{H},-0.4176362805,1.3526891$ $948,2.5283817415 \backslash \mathrm{H}, 1.1692015256,2.4478261733,3.778394386 \backslash 0,3.195121781$ $6,2.5504248629,-0.7265206982 \backslash \mathrm{H}, 3.3386060862,3.1824927344,-1.4504066994$ $\backslash \mathrm{H}, 2.6927519881,-0.9154123005,-1.8837614547 \backslash \mathrm{H},-4.8129603795,-2.0777890$ $386,1.3733887396 \backslash \mathrm{H},-4.8615635147,-4.5622048609,-0.4858990757 \backslash \mathrm{H},-6.2187$ $517042,-4.0895954882,0.5706707494 \backslash \mathrm{H},-4.7351719298,-4.7043548784,1.2841$ $939967 \backslash \mathrm{H},-2.9327458321,-0.5878482215,-2.3839128435 \backslash \mathrm{H},-2.081673497,1.78$ $8079338,-2.0254547294 \backslash \mathrm{H},-3.7961033371,1.4790913051,-1.760263303 \backslash \mathrm{H},-4.7$ $985940872,1.726425776,0.7599261276 \backslash \mathrm{H}, 0.0869055767,2.537185819,-0.70683$ $37587 \backslash \mathrm{H}, 1.5410000115,3.8646703404,0.80082383 \backslash \mathrm{H}, 0.6881933939,4.70874032$ $96,2.9700825821 \backslash \mathrm{H},-1.6293934798,4.2004912969,3.7106800283 \backslash \mathrm{H}, 0.57645946$ $1,-3.4075909791,-1.2403919598 \backslash \mathrm{H}, 1.1840586485,-1.2609146229,-3.35792716$ $89 \backslash \mathrm{H}, 0.5488430257,-2.8821878755,-3.6666993213 \backslash \mathrm{H}, 2.188028897,-2.7078956$ $126,-3.0393105651 \backslash \mathrm{H}, 2.1417469169,0.56853638,0.4811739572 \backslash \mathrm{H}, 5.035127590$ $7,-0.1892864108,-0.2072883139 \backslash \mathrm{H}, 4.5842518664,1.0883687397,0.9175829233$ $\backslash \mathrm{H}, 5.3746999931,-1.1386236753,1.9111745297 \backslash \mathrm{H}, 3.8810767323,-1.869575563$ $5,1.3563717272 \backslash \mathrm{H}, 3.9558005449,-1.1270485058,3.8083476338 \backslash \mathrm{H}, 4.004767432$ $1,0.5480795833,3.2346765744 \backslash \mathrm{H}, 2.509188163,1.5575469807,4.4365665288 \backslash \backslash \mathrm{V}$ ersion=EM64L-G0 9RevA. 02 $\backslash \mathrm{HF}=-1655.0045986 \backslash \mathrm{S} 2=0.756985 \backslash \mathrm{S} 2-1=0 . \backslash \mathrm{S} 2 \mathrm{~A}=0.750$ $04 \backslash \mathrm{RMSD}=4.005 e-09 \backslash \mathrm{RMSF}=5.212 e-06 \backslash \mathrm{Dipole}=0.5913092,-0.8505528,3.4245018$ $\backslash \mathrm{PG}=\mathrm{C} 01 \quad[\mathrm{X}(\mathrm{C} 23 \mathrm{H} 33 \mathrm{~N} 705)]$

\section{Table S5. M06-2X/6-31+G(d,p) Optimized Geometry of AWAR Radical $\mathbf{1 b .}$}

\footnotetext{
$\mathrm{C},-0.1905455814,-1.4507786211$

$,-3.4809260999 \backslash \mathrm{C},-0.646067759,-0.8682778609,-2.2235531821 \backslash 0,-0.1634919$ $984,0.1646546148,-1.7498652765 \backslash \mathrm{C}, 0.7971110186,-0.7415941148,-4.3325063$ $787 \backslash \mathrm{N},-1.6681699661,-1.5221325382,-1.5817883947 \backslash \mathrm{C},-2.0910252036,-1.054$ $8922724,-0.2835095044 \backslash \mathrm{C},-0.8873270215,-0.9443372514,0.6551855213 \backslash 0,-0$. $0479703682,-1.8415798129,0.7154180295 \backslash \mathrm{C},-3.1268854409,-2.0145457759,0$. $3291122058 \backslash \mathrm{C},-3.450344809,-1.6377812796,1.7421526195 \backslash \mathrm{C},-4.0214590009,-$ $0.3938521662,2.1934148479 \backslash \mathrm{C},-4.022977633,-0.4260838583,3.608285695 \backslash \mathrm{N},-$ $3.4798333682,-1.6331431166,3.9913959051 \backslash \mathrm{C},-3.1426023066,-2.3544769782$, $2.8667908441 \backslash \mathrm{C},-4.5241170972,0.7442664705,1.5366641415 \backslash \mathrm{C},-4.9980835001$ $, 1.8021966095,2.2960716671 \backslash \mathrm{C},-4.9878460387,1.7472730687,3.7052843231 \backslash \mathrm{C}$ $,-4.5066446128,0.6365839787,4.3799038211 \backslash \mathrm{H},-3.4363771002,-1.9787690002$

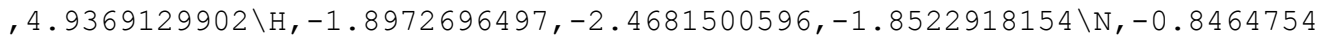
$225,0.1753139978,1.407062322 \backslash C, 0.1323229193,0.4580891671,2.4483888519 \backslash$ C, $1.4962818546,0.8251433105,1.8541763533 \backslash 0,2.0303035085,1.9076718349,2$
} 
$.1702583101 \backslash \mathrm{C}, 0.2320991665,-0.6519969912,3.4993959493 \backslash \mathrm{H},-1.5741003901$, $0.8619797365,1.2623512955 \backslash \mathrm{N}, 2.0632813036,-0.0600342187,1.0286425305 \backslash \mathrm{C}$, $3.2276351162,0.243322074,0.2092706192 \backslash \mathrm{C}, 2.9389701894,-0.1240563444,-1$. $2415260704 \backslash 0,3.2161731023,0.5757118408,-2.1802849698 \backslash \mathrm{C}, 4.4896418046,-0$ $.5222263457,0.6561633476 \backslash \mathrm{C}, 5.0416782103,-0.1715277292,2.041608132 \backslash \mathrm{C}, 5$. $7300857742,1.1922713968,2.1438214766 \backslash \mathrm{N}, 4.7246504505,2.2519524439,2.152$ $6264686 \backslash \mathrm{C}, 4.8485223725,3.4661477053,1.6454622133 \backslash \mathrm{N}, 6.0511545774,3.9734$ $375571,1.3363652919 \backslash \mathrm{N}, 3.7410113801,4.1843500715,1.4220607258 \backslash \mathrm{H}, 3.75071$ $62826,1.9867534581,2.367411287 \backslash \mathrm{H}, 2.8374645769,3.7449992678,1.578232528$ $6 \backslash \mathrm{H}, 3.782708444,5.1772814326,1.2565002713 \backslash \mathrm{H}, 6.1373235168,4.8374227433$, $0.8243275866 \backslash 0,2.4161728829,-1.3525951602,-1.3320031158 \backslash \mathrm{H}, 2.1876749617$ $,-1.5299553296,-2.258997419 \backslash \mathrm{H}, 1.5244323277,-0.9083559546,0.8083705433 \backslash$ $\mathrm{H},-0.6505153696,-2.3696571408,-3.8331668401 \backslash \mathrm{H}, 1.2774027673,0.071982361$ $8,-3.782881446 \backslash \mathrm{H}, 0.2991389941,-0.3040825015,-5.2091698949 \backslash \mathrm{H}, 1.55588097$ $33,-1.4258466051,-4.7302837276 \backslash \mathrm{H},-2.5312291014,-0.0571506109,-0.401739$ $9063 \backslash \mathrm{H},-4.0220483676,-2.0033908288,-0.3043840115 \backslash \mathrm{H},-2.7113611184,-3.02$ $90172409,0.3108500757 \backslash \mathrm{H},-2.6894528887,-3.333023031,2.9509965715 \backslash \mathrm{H},-4.5$ $671439372,0.7869395733,0.4511450328 \backslash \mathrm{H},-5.398221526,2.681938903,1.80278$ $46596 \backslash \mathrm{H},-5.374321516,2.5875603392,4.2724997265 \backslash \mathrm{H},-4.5098134549,0.59256$ $89181,5.4647024411 \backslash \mathrm{H},-0.2025066899,1.3744460057,2.9384034347 \backslash \mathrm{H}, 0.59164$ $22247,-1.5850142297,3.0635762526 \backslash \mathrm{H},-0.7641661362,-0.8226248002,3.91795$ $5867 \backslash \mathrm{H}, 0.9051980702,-0.3397207586,4.3027542135 \backslash \mathrm{H}, 3.3859856996,1.322632$ $0624,0.2169660748 \backslash \mathrm{H}, 4.2456122537,-1.589535209,0.635990219 \backslash \mathrm{H}, 5.26906988$ $2,-0.3612813232,-0.0994739397 \backslash \mathrm{H}, 5.7815509028,-0.9298523793,2.314132684$ $8 \backslash \mathrm{H}, 4.2478373983,-0.2372039423,2.7982198468 \backslash \mathrm{H}, 6.3282572349,1.242714352$ $5,3.0604800623 \backslash \mathrm{H}, 6.3979964034,1.3351220305,1.2849124795 \backslash \mathrm{H}, 6.8975188287$ , 3.5168560548,1.6373276355 \Version=EM64L-G09RevA.02\HF=-1654.9936129 \ $\mathrm{S} 2=0.757052 \backslash \mathrm{S} 2-1=0 . \backslash \mathrm{S} 2 \mathrm{~A}=0.75004 \backslash \mathrm{RMSD}=6.218 \mathrm{e}-09 \backslash \mathrm{RMSF}=4.606 \mathrm{e}-06 \backslash \mathrm{Dipole}=3$ $.8956199,1.2157026,-3.1625491 \backslash \mathrm{PG}=\mathrm{C} 01 \quad[\mathrm{X}(\mathrm{C} 23 \mathrm{H} 33 \mathrm{~N} 705)] \backslash \backslash @$

Table S6. M06-2X/6-31+G(d,p) Optimized Geometry of AWAR Radical 1c.

AAWAR z4 trans cation-radical, stacked $\backslash \backslash 1,2 \backslash C,-2.65899$

$5638,1.3283010546,2.5551763055 \backslash \mathrm{C},-2.1424515899,0.170345465,1.845484756$ $5 \backslash 0,-1.5542522536,-0.7602299818,2.4189057301 \backslash \mathrm{C},-2.5000644497,1.4608966$ $058,4.0221556518 \backslash \mathrm{N},-2.3291969312,0.1615169261,0.4868758345 \backslash \mathrm{C},-2.237035$ $1002,-1.0760149069,-0.2808551646 \backslash \mathrm{C},-0.7855386501,-1.345347531,-0.71786$ $15792 \backslash 0,-0.4627335099,-1.3777821699,-1.9086833987 \backslash \mathrm{C},-3.1624572495,-1.0$ $198647509,-1.5040498347 \backslash \mathrm{C},-4.5710107088,-0.6581649785,-1.138651752 \backslash \mathrm{C},-$ $5.1137708237,0.6756075624,-1.0768769129 \backslash \mathrm{C},-6.4451014897,0.5671005129,-$ $0.6083619455 \backslash N,-6.6964939466,-0.7695277494,-0.4085357323 \backslash \mathrm{C},-5.57270860$ $32,-1.4977817415,-0.7276496994 \backslash \mathrm{C},-4.5991076624,1.951368354,-1.37581055$ $94 \backslash \mathrm{C},-5.4031217257,3.0632983022,-1.1832388245 \backslash \mathrm{C},-6.7231404725,2.929587$ $9337,-0.704023404 \backslash \mathrm{C},-7.2631176022,1.6863280972,-0.4170557337 \backslash \mathrm{H},-7.5729$ $758924,-1.15974644,-0.101215353 \backslash \mathrm{H},-2.9548178662,0.8660285495,0.1149219$ $422 \backslash \mathrm{N}, 0.0834642623,-1.5369832211,0.2956161516 \backslash \mathrm{C}, 1.4656641081,-1.980482$ $5131,0.1613976267 \backslash \mathrm{C}, 2.4210217592,-0.9330131385,-0.4119016108 \backslash 0,3.54141$ $04092,-0.7760569686,0.1157648489 \backslash \mathrm{C}, 1.6067631484,-3.2904485965,-0.62690$ $04574 \backslash \mathrm{H},-0.3038100893,-1.4350908334,1.2387926567 \backslash \mathrm{N}, 2.0324065385,-0.278$ $2201563,-1.5100264365 \backslash C, 2.7865572525,0.8025392337,-2.110763903 \backslash C, 1.951$ $6052269,2.0704299927,-2.2646586831 \backslash 0,2.4332492834,3.1175302137,-2.6136$ $122811 \backslash \mathrm{C}, 3.3521507078,0.4302021654,-3.4974482086 \backslash \mathrm{C}, 4.36636614,-0.71757$ $23247,-3.5140955817 \backslash \mathrm{C}, 5.7490830728,-0.3749501449,-2.9528331145 \backslash \mathrm{N}, 5.690$ $6827642,-0.3063742041,-1.4945777857 \backslash \mathrm{C}, 6.4893142148,0.3891807669,-0.704$ $0135033 \backslash \mathrm{N}, 7.626955365,0.9277430191,-1.1653927308 \backslash \mathrm{N}, 6.1302882413,0.5703$ $124808,0.5737923594 \backslash \mathrm{H}, 4.8670553852,-0.7001289786,-1.0160695733 \backslash \mathrm{H}, 5.233$ $9934321,0.2026431056,0.8826100749 \backslash \mathrm{H}, 6.7939649141,0.8826153149,1.264766$ $0555 \backslash \mathrm{H}, 8.1693261743,1.5649189553,-0.6031461939 \backslash 0,0.6513976958,1.891794$ $1147,-2.0045436283 \backslash \mathrm{H}, 0.1998614383,2.7378670894,-2.1562340294 \backslash \mathrm{H}, 1.11068$ $22101,-0.5243669375,-1.8954042111 \backslash \mathrm{H},-3.1871166131,2.0921587222,1.99044$ $09775 \backslash \mathrm{H},-3.477189695,1.547194922,4.512323849 \backslash \mathrm{H},-1.945071022,2.37278119$ $26,4.2752311471 \backslash \mathrm{H},-1.9729577711,0.5959939409,4.4274543682 \backslash \mathrm{H},-2.5415107$ $82,-1.899670047,0.3802471742 \backslash \mathrm{H},-2.7548208323,-0.2976309244,-2.22005828$ 
$68 \backslash \mathrm{H},-3.1195553543,-1.9916542514,-2.0030574069 \backslash \mathrm{H},-5.5743514413,-2.5758$ $075562,-0.6406383809 \backslash \mathrm{H},-3.5935822619,2.0639402469,-1.7776845972 \backslash \mathrm{H},-5.0$ $213289932,4.0519040695,-1.4165817046 \backslash \mathrm{H},-7.3313552289,3.8174258188,-0.5$ $666863866 \backslash \mathrm{H},-8.2826158096,1.5866934727,-0.0576117638 \backslash \mathrm{H}, 1.8281662922,-2$ $.1427525979,1.1784527237 \backslash \mathrm{H}, 1.2504992312,-3.1722262817,-1.6516804421 \backslash \mathrm{H}$, $1.0196993456,-4.070470849,-0.1375044063 \backslash \mathrm{H}, 2.6548266861,-3.6029888712,-$ $0.6382438613 \backslash \mathrm{H}, 3.5951657809,1.0734101434,-1.4267181858 \backslash \mathrm{H}, 2.5052605316$, $0.1521599401,-4.1356338629 \backslash \mathrm{H}, 3.7967523274,1.332166445,-3.9317154645 \backslash \mathrm{H}$, $4.5081721968,-1.0295664078,-4.5527587938 \backslash \mathrm{H}, 3.9659797032,-1.594609642,-$ $2.9872519745 \backslash \mathrm{H}, 6.4736181877,-1.1388056089,-3.2577738486 \backslash \mathrm{H}, 6.0773098533$ $, 0.5952431024,-3.3472898515 \backslash \mathrm{H}, 7.9720381795,0.6994914726,-2.0843591893 \backslash$ $\backslash$ Version=EM64L-G0 9RevA. 02 $\backslash \mathrm{HF}=-1654.9909349 \backslash \mathrm{S} 2=0.756981 \backslash \mathrm{S} 2-1=0 . \backslash \mathrm{S} 2 \mathrm{~A}=0.7$ $50039 \backslash \mathrm{RMSD}=6.592 \mathrm{e}-09 \backslash \mathrm{RMSF}=3.489 \mathrm{e}-06 \backslash \mathrm{Dipole}=-6.0899139,0.335073,0.90284$ $07 \backslash P G=C 01 \quad[X(C 23 H 33 N 705)] \backslash \backslash @$

Table S7. M06-2X/6-31+G(d,p) Optimized Geometry of AWAR Radical 1d.

AAWAR z4 ion, new conformer, COOH close to Trp \\1,2\C,-

$1.808286189,-3.7736629183,2.1339570285 \backslash C,-1.7596677381,-3.4118395903,0$ $.726174984 \backslash 0,-1.0300528095,-3.9943257562,-0.0906160162 \backslash \mathrm{C},-0.9857317936$ $,-4.8854672497,2.6651411301 \backslash \mathrm{N},-2.5600567169,-2.3731601081,0.3388908073$ $\backslash \mathrm{C},-2.7823674017,-2.0020958402,-1.0455857081 \backslash \mathrm{C},-1.6649600551,-1.111119$ $7338,-1.6182835029 \backslash 0,-1.9195608616,-0.1365939285,-2.3284490661 \backslash \mathrm{C},-4.17$ $28659032,-1.3514946878,-1.2053939194 \backslash C,-4.4663730232,-0.2634240955,-0$. $2105207107 \backslash \mathrm{C},-4.2232275295,1.1563858617,-0.3220132581 \backslash \mathrm{C},-4.6161709718$, $1.7413525812,0.9065169579 \backslash \mathrm{N},-5.0934844696,0.7363918906,1.713241031 \backslash \mathrm{C},-$ $5.0196857196,-0.4576848124,1.0321628938 \backslash C,-3.7693887666,1.9996741707,-$ $1.3549291095 \backslash \mathrm{C},-3.678439498,3.3612813872,-1.1185039702 \backslash \mathrm{C},-4.0217093306$ , 3.914627162,0.1344302781\C, $-4.4990062979,3.1164174057,1.1613409971 \backslash \mathrm{H}$, $-5.5242179149,0.8665759613,2.6149221755 \backslash \mathrm{H},-3.027743471,-1.8105387255,1$ $.0383034761 \backslash \mathrm{N},-0.4060021088,-1.4881072756,-1.3102476021 \backslash \mathrm{C}, 0.8052221384$ $,-0.9225025982,-1.8969726987 \backslash \mathrm{C}, 1.1594811965,0.4818745509,-1.4050976282$ $\backslash 0,2.3528378616,0.7378781818,-1.1375713603 \backslash \mathrm{C}, 0.8213233004,-0.948413628$ $9,-3.4326824382 \backslash \mathrm{H},-0.3109071224,-2.3952214777,-0.8514170582 \backslash \mathrm{N}, 0.190701$ $339,1.3982287353,-1.357253512 \backslash \mathrm{C}, 0.3782824669,2.7545951461,-0.878101843$ $6 \backslash C,-0.6429137904,3.1275838943,0.2002822497 \backslash 0,-0.7943207087,4.26637297$ $42,0.566132049 \backslash \mathrm{C}, 0.2878707523,3.7914890436,-2.0134385727 \backslash \mathrm{C}, 1.357382824$ $6,3.6973995645,-3.1054194504 \backslash C, 2.7573601804,4.1591016167,-2.6911462114$ $\backslash N, 3.3837406307,3.1380967953,-1.8555281755 \backslash \mathrm{C}, 4.3754341792,3.3109558968$ $,-1.0017758618 \backslash \mathrm{N}, 5.0840972433,4.4487895263,-0.9767342645 \backslash \mathrm{N}, 4.652382598$ $1,2.3290045072,-0.1315478432 \backslash \mathrm{H}, 2.9409110938,2.2069892719,-1.8024623684$ $\backslash \mathrm{H}, 4.0732149612,1.4938531524,-0.1463716243 \backslash \mathrm{H}, 5.5310739026,2.3012702493$ $, 0.3608506811 \backslash \mathrm{H}, 5.7498295949,4.6338019247,-0.2428485028 \backslash 0,-1.309379508$ $2,2.0813568682,0.6852517188 \backslash \mathrm{H},-2.0359973611,2.4184167247,1.2424876706 \backslash$ $\mathrm{H},-0.7452126988,1.1056020496,-1.6565967624 \backslash \mathrm{H},-2.4682517508,-3.21867707$ $48,2.7948407879 \backslash \mathrm{H},-0.3926086591,-5.331930973,1.8659177334 \backslash \mathrm{H},-1.6203897$ $661,-5.6594593769,3.1133345643 \backslash \mathrm{H},-0.3162794428,-4.5348024784,3.4601678$ $699 \backslash \mathrm{H},-2.7523414633,-2.9283936438,-1.6352587218 \backslash \mathrm{H},-4.2509331831,-0.965$ $7844921,-2.2246542753 \backslash \mathrm{H},-4.9131451592,-2.149861637,-1.0959083419 \backslash \mathrm{H},-5$. $4010662883,-1.3686232675,1.4763726697 \backslash \mathrm{H},-3.4979846005,1.5798143207,-2$. $316789876 \backslash \mathrm{H},-3.3445563431,4.0250064571,-1.9104826479 \backslash \mathrm{H},-3.9144917962,4$ $.9820650683,0.2947017468 \backslash \mathrm{H},-4.7948590243,3.5423533197,2.1152819947 \backslash \mathrm{H}, 1$ $.6267310698,-1.5414406318,-1.531117865 \backslash \mathrm{H}, 0.0273241775,-0.3247242531,-3$ $.8461765529 \backslash \mathrm{H}, 0.6791245958,-1.9751766177,-3.7769660439 \backslash \mathrm{H}, 1.7911308527$, $-0.594830242,-3.7942203011 \backslash \mathrm{H}, 1.3541355578,2.798673909,-0.3829437814 \backslash \mathrm{H}$, $-0.6967083542,3.670921973,-2.4812515566 \backslash \mathrm{H}, 0.296852241,4.7867471309,-1$. $5584963831 \backslash \mathrm{H}, 1.043473505,4.331916743,-3.9391951659 \backslash \mathrm{H}, 1.4164089127,2.67$ $69378742,-3.5091729392 \backslash \mathrm{H}, 3.3708568616,4.3327922184,-3.5830263528 \backslash \mathrm{H}, 2.6$ $859256389,5.0981939363,-2.1269306718 \backslash \mathrm{H}, 4.9593659143,5.1514550694,-1.68$ $84359786 \backslash \backslash$ Version=EM64 L-G0 9RevA. $02 \backslash \mathrm{HF}=-1654.9974958 \backslash \mathrm{S} 2=0.756849 \backslash \mathrm{S} 2-1=0$ $. \backslash \mathrm{S} 2 \mathrm{~A}=0.750038 \backslash \mathrm{RMSD}=4.604 \mathrm{e}-09 \backslash \mathrm{RMSF}=3.997 \mathrm{e}-06 \backslash \mathrm{Dipole}=-5.2615592,-1.0498$ $432,0.5533772 \backslash P G=C 01 \quad[X(C 23 H 33 N 705)] \backslash \backslash 0$ 
Table S8. M06-2X/6-31+G(d,p) Optimized Geometry of AWAR Radical 2a.

\# um062x/6-31+G(d,p) scf=(xqc, tight) pop=none opt caches ize=131072 \\AAWAR z4 ion, new Trp-2 alpha radical conformer $\backslash \backslash 1,2 \backslash C,-3$. $6683745343,-4.1551902204,-0.5417454087 \backslash C,-2.3272560651,-3.6460339814,-$ $1.036176992 \backslash 0,-1.32261586,-4.3370467039,-1.0554611575 \backslash \mathrm{C},-3.5730135431$, $-5.5708721829,0.0109775342 \backslash N,-2.3491327103,-2.3342840882,-1.4786524452$ $\backslash \mathrm{C},-1.3851522466,-1.5575386876,-2.1139289397 \backslash \mathrm{C}, 0.0346437597,-1.4692827$ $835,-1.7530346656 \backslash 0,0.6921302594,-0.4901975405,-2.1499454792 \backslash C,-1.9410$ $747431,-0.5041416588,-3.0347152374 \backslash \mathrm{C},-2.6432901401,0.6117172819,-2.304$ $0722082 \backslash \mathrm{C},-2.0387023927,1.7987529213,-1.749140146 \backslash \mathrm{C},-3.0823823518,2.56$ $3038317,-1.1685354635 \backslash \mathrm{N},-4.2533291295,1.8676436861,-1.3480013177 \backslash \mathrm{C},-3$. $9865732863,0.7107457538,-2.0489967585 \backslash \mathrm{C},-0.7288281791,2.3166245443,-1$. $753126064 \backslash \mathrm{C},-0.5016353965,3.5642787581,-1.1933186745 \backslash \mathrm{C},-1.5563417086,4$ $.3092830044,-0.6207960712 \backslash \mathrm{C},-2.8553029326,3.8203151536,-0.5874182467 \backslash \mathrm{H}$ $,-5.1746888742,2.202320197,-1.1130646841 \backslash \mathrm{H},-3.2704414806,-1.9064191678$ $,-1.4750687068 \backslash \mathrm{N}, 0.5940839651,-2.395617033,-0.937605416 \backslash \mathrm{C}, 1.9617328844$ $,-2.2109001471,-0.4859740653 \backslash \mathrm{C}, 2.0468985227,-0.7747072389,0.0494631864$ $\backslash 0,1.1969967235,-0.3821850181,0.8587522113 \backslash \mathrm{C}, 2.9912607117,-2.572879425$ $5,-1.5557745078 \backslash \mathrm{H}, 0.1149500673,-3.2801471347,-0.7864470182 \backslash \mathrm{N}, 3.0577616$ $118,0.0091730118,-0.3747730232 \backslash \mathrm{C}, 2.8981028662,1.455438473,-0.413530916$ $1 \backslash \mathrm{C}, 3.1801282164,1.8840821013,-1.8485731335 \backslash 0,3.7850807564,1.217142158$ $6,-2.647385499 \backslash C, 3.8598020651,2.2159939458,0.5222139503 \backslash C, 3.6709844686$ $, 1.9830021673,2.0252858651 \backslash \mathrm{C}, 2.4433307876,2.6625409026,2.6392271587 \backslash \mathrm{N}$, $1.2394784863,1.9230907379,2.2759647313 \backslash \mathrm{C}, 0.0459605256,2.4318868551,2.0$ $106842696 \backslash \mathrm{N},-0.2521189334,3.7015061872,2.3185141854 \backslash \mathrm{N},-0.8833509619,1$. $6448733669,1.4508823084 \backslash \mathrm{H}, 1.3669353727,0.9581585089,1.9389521779 \backslash \mathrm{H},-0$. $5562322563,0.7893224059,1.007413558 \backslash \mathrm{H},-1.7076097358,2.0746579273,1.046$ $9335833 \backslash \mathrm{H},-1.1260923888,4.102604898,2.0131134165 \backslash 0,2.7135089844,3.1155$ $116073,-2.0992887575 \backslash \mathrm{H}, 2.9560544962,3.3525428798,-3.0097524422 \backslash \mathrm{H}, 3.603$ $635143,-0.3458889124,-1.1548722125 \backslash \mathrm{H},-4.0456875766,-3.4599243167,0.218$ $3556245 \backslash \mathrm{H},-3.1926593209,-6.256531757,-0.7482229565 \backslash \mathrm{H},-4.5571549374,-5$. $9157971524,0.3337981018 \backslash \mathrm{H},-2.8926535245,-5.6100110935,0.8641696356 \backslash \mathrm{H},-$ $1.1154717204,-0.0967672805,-3.6214043957 \backslash \mathrm{H},-2.6436903344,-0.9829603302$ $,-3.7278254384 \backslash \mathrm{H},-4.7960121453,0.0581737805,-2.3504088483 \backslash \mathrm{H}, 0.07810413$ $54,1.7352127233,-2.1914484177 \backslash \mathrm{H}, 0.5008449209,3.981837841,-1.2106144802$ $\backslash \mathrm{H},-1.3531160043,5.3029437936,-0.2300864445 \backslash \mathrm{H},-3.6712103856,4.41002751$ $22,-0.1795479426 \backslash \mathrm{H}, 2.0908817665,-2.8592063049,0.3876670047 \backslash \mathrm{H}, 2.8700163$ $331,-1.9392079496,-2.4393298843 \backslash \mathrm{H}, 2.8389304063,-3.6110238187,-1.857200$ $5683 \backslash \mathrm{H}, 4.0135956039,-2.4879078559,-1.1734287868 \backslash \mathrm{H}, 1.8516993249,1.69767$ $78539,-0.1958732076 \backslash \mathrm{H}, 4.8787647719,1.9185257818,0.2497727726 \backslash \mathrm{H}, 3.77136$ $95813,3.2870830418,0.3042451935 \backslash \mathrm{H}, 4.5524455247,2.3760468233,2.54031106$ $92 \backslash \mathrm{H}, 3.6437460015,0.9076784842,2.2454258264 \backslash \mathrm{H}, 2.5421370511,2.702436512$ $4,3.72961202 \backslash \mathrm{H}, 2.3693264029,3.6900220841,2.2607194562 \backslash \mathrm{H}, 0.3542431728,4$ $.2546533328,2.9019434741 \backslash \mathrm{H},-4.3745748707,-4.1132325329,-1.3810602861 \backslash \backslash$ Version=EM64L-G09RevA. 02 \HF=-1655.0088001 \S2=0.75699 $S 2-1=0 . \backslash S 2 A=0.750$ $039 \backslash \mathrm{RMSD}=6.112 \mathrm{e}-09 \backslash \mathrm{RMSF}=3.772 \mathrm{e}-06 \backslash \mathrm{Dipole}=-3.148916,0.3171377,2.0322168$ $\backslash \mathrm{PG}=\mathrm{C} 01 \quad[\mathrm{X}(\mathrm{C} 23 \mathrm{H} 33 \mathrm{~N} 705)] \backslash \backslash \mathrm{Q}$

Table S9. M06-2X/6-31+G(d,p) Optimized Geometry of AWAR Radical 2b.

\# um062x/6-31+G(d,p) scf=(xqc, tight) pop=none opt caches ize=131072 \\AAWAR z4 ion, new Trp-2 alpha radical conformer $\backslash \backslash 1,2 \backslash C,-3$. $6683745343,-4.1551902204,-0.5417454087 \backslash \mathrm{C},-2.3272560651,-3.6460339814,-$ $1.036176992 \backslash 0,-1.32261586,-4.3370467039,-1.0554611575 \backslash \mathrm{C},-3.5730135431$, $-5.5708721829,0.0109775342 \backslash N,-2.3491327103,-2.3342840882,-1.4786524452$ $\backslash C,-1.3851522466,-1.5575386876,-2.1139289397 \backslash C, 0.0346437597,-1.4692827$ $835,-1.7530346656 \backslash 0,0.6921302594,-0.4901975405,-2.1499454792 \backslash \mathrm{C},-1.9410$ $747431,-0.5041416588,-3.0347152374 \backslash C,-2.6432901401,0.6117172819,-2.304$ $0722082 \backslash \mathrm{C},-2.0387023927,1.7987529213,-1.749140146 \backslash \mathrm{C},-3.0823823518,2.56$ $3038317,-1.1685354635 \backslash \mathrm{N},-4.2533291295,1.8676436861,-1.3480013177 \backslash \mathrm{C},-3$. $9865732863,0.7107457538,-2.0489967585 \backslash \mathrm{C},-0.7288281791,2.3166245443,-1$. $753126064 \backslash \mathrm{C},-0.5016353965,3.5642787581,-1.1933186745 \backslash \mathrm{C},-1.5563417086,4$ 
$.3092830044,-0.6207960712 \backslash \mathrm{C},-2.8553029326,3.8203151536,-0.5874182467 \backslash \mathrm{H}$ $,-5.1746888742,2.202320197,-1.1130646841 \backslash \mathrm{H},-3.2704414806,-1.9064191678$ $,-1.4750687068 \backslash \mathrm{N}, 0.5940839651,-2.395617033,-0.937605416 \backslash \mathrm{C}, 1.9617328844$ $,-2.2109001471,-0.4859740653 \backslash \mathrm{C}, 2.0468985227,-0.7747072389,0.0494631864$ $\backslash 0,1.1969967235,-0.3821850181,0.8587522113 \backslash \mathrm{C}, 2.9912607117,-2.572879425$ $5,-1.5557745078 \backslash \mathrm{H}, 0.1149500673,-3.2801471347,-0.7864470182 \backslash \mathrm{N}, 3.0577616$ $118,0.0091730118,-0.3747730232 \backslash C, 2.8981028662,1.455438473,-0.413530916$ $1 \backslash \mathrm{C}, 3.1801282164,1.8840821013,-1.8485731335 \backslash 0,3.7850807564,1.217142158$ $6,-2.647385499 \backslash C, 3.8598020651,2.2159939458,0.5222139503 \backslash C, 3.6709844686$ $, 1.9830021673,2.0252858651 \backslash \mathrm{C}, 2.4433307876,2.6625409026,2.6392271587 \backslash \mathrm{N}$, $1.2394784863,1.9230907379,2.2759647313 \backslash \mathrm{C}, 0.0459605256,2.4318868551,2.0$ $106842696 \backslash \mathrm{N},-0.2521189334,3.7015061872,2.3185141854 \backslash \mathrm{N},-0.8833509619,1$. $6448733669,1.4508823084 \backslash \mathrm{H}, 1.3669353727,0.9581585089,1.9389521779 \backslash \mathrm{H},-0$. $5562322563,0.7893224059,1.007413558 \backslash \mathrm{H},-1.7076097358,2.0746579273,1.046$ $9335833 \backslash \mathrm{H},-1.1260923888,4.102604898,2.0131134165 \backslash 0,2.7135089844,3.1155$ $116073,-2.0992887575 \backslash \mathrm{H}, 2.9560544962,3.3525428798,-3.0097524422 \backslash \mathrm{H}, 3.603$ $635143,-0.3458889124,-1.1548722125 \backslash \mathrm{H},-4.0456875766,-3.4599243167,0.218$ $3556245 \backslash \mathrm{H},-3.1926593209,-6.256531757,-0.7482229565 \backslash \mathrm{H},-4.5571549374,-5$. $9157971524,0.3337981018 \backslash \mathrm{H},-2.8926535245,-5.6100110935,0.8641696356 \backslash \mathrm{H},-$ $1.1154717204,-0.0967672805,-3.6214043957 \backslash \mathrm{H},-2.6436903344,-0.9829603302$ $,-3.7278254384 \backslash \mathrm{H},-4.7960121453,0.0581737805,-2.3504088483 \backslash \mathrm{H}, 0.07810413$ $54,1.7352127233,-2.1914484177 \backslash \mathrm{H}, 0.5008449209,3.981837841,-1.2106144802$ $\backslash \mathrm{H},-1.3531160043,5.3029437936,-0.2300864445 \backslash \mathrm{H},-3.6712103856,4.41002751$ $22,-0.1795479426 \backslash \mathrm{H}, 2.0908817665,-2.8592063049,0.3876670047 \backslash \mathrm{H}, 2.8700163$ $331,-1.9392079496,-2.4393298843 \backslash \mathrm{H}, 2.8389304063,-3.6110238187,-1.857200$ $5683 \backslash \mathrm{H}, 4.0135956039,-2.4879078559,-1.1734287868 \backslash \mathrm{H}, 1.8516993249,1.69767$ $78539,-0.1958732076 \backslash \mathrm{H}, 4.8787647719,1.9185257818,0.2497727726 \backslash \mathrm{H}, 3.77136$ $95813,3.2870830418,0.3042451935 \backslash \mathrm{H}, 4.5524455247,2.3760468233,2.54031106$ $92 \backslash \mathrm{H}, 3.6437460015,0.9076784842,2.2454258264 \backslash \mathrm{H}, 2.5421370511,2.702436512$ $4,3.72961202 \backslash \mathrm{H}, 2.3693264029,3.6900220841,2.2607194562 \backslash \mathrm{H}, 0.3542431728,4$ $.2546533328,2.9019434741 \backslash \mathrm{H},-4.3745748707,-4.1132325329,-1.3810602861 \backslash \backslash$ Version=EM64L-G09RevA. 02 \HF=-1655.0088001 \S2=0.75699 \S2-1=0. \S2A=0.750 $039 \backslash \mathrm{RMS} D=6.112 \mathrm{e}-09 \backslash \mathrm{RMSF}=3.772 \mathrm{e}-06 \backslash \mathrm{Dipole}=-3.148916,0.3171377,2.0322168$ $\backslash \mathrm{PG}=\mathrm{C} 01 \quad[\mathrm{X}(\mathrm{C} 23 \mathrm{H} 33 \mathrm{~N} 705)] \backslash \backslash \mathrm{C}$

Table S10. M06-2X/6-31+G(d,p) Optimized Geometry of AWAR Radical 3a.

\# um062x/6-311++G(2d,p) scf=(xqc, tight) pop=npa

td(Nstates=20) cachesize=131072 \\AAWAR z4 Ala-3 alpha radical, conform er $\backslash \backslash 1,2 \backslash C, 0,-4.187255,-2.660484,1.921837 \backslash C, 0,-3.648559,-2.463706,0.519$ $331 \backslash 0,0,-3.453515,-3.400351,-0.252545 \backslash C, 0,-5.026155,-3.929274,2.036409$ $\backslash \mathrm{N}, 0,-3.363326,-1.180701,0.158728 \backslash \mathrm{C}, 0,-2.849056,-0.844386,-1.158779 \backslash \mathrm{C}$, $0,-1.324222,-1.010703,-1.198604 \backslash 0,0,-0.553746,-0.094604,-1.42442 \backslash \mathrm{C}, 0,-$ $3.315509,0.546677,-1.60931 \backslash C, 0,-2.986028,1.695639,-0.69756 \backslash C, 0,-1.9703$ $56,2.69723,-0.904938 \backslash \mathrm{C}, 0,-2.068063,3.626133,0.160526 \backslash \mathrm{N}, 0,-3.093349,3.2$ $09383,0.973371 \backslash \mathrm{C}, 0,-3.654679,2.068872,0.442152 \backslash \mathrm{C}, 0,-1.015857,2.913824$, $-1.914512 \backslash \mathrm{C}, 0,-0.192626,4.026782,-1.83321 \backslash \mathrm{C}, 0,-0.290512,4.928127,-0.74$ $7801 \backslash \mathrm{C}, 0,-1.223605,4.739589,0.263673 \backslash \mathrm{H}, 0,-3.439777,3.703644,1.780393 \backslash \mathrm{H}$ $, 0,-3.378979,-0.449005,0.857769 \backslash \mathrm{N}, 0,-0.925957,-2.318747,-0.983516 \backslash \mathrm{C}, 0$, $0.371647,-2.757942,-0.854559 \backslash \mathrm{C}, 0,1.302789,-1.912367,-0.126631 \backslash 0,0,0.89$ $0581,-1.046464,0.667418 \backslash \mathrm{C}, 0,0.72252,-4.101477,-1.402398 \backslash \mathrm{H}, 0,-1.679032$, $-3.012917,-0.979455 \backslash \mathrm{N}, 0,2.636486,-2.124691,-0.335692 \backslash \mathrm{C}, 0,3.614562,-1.0$ $66235,-0.122955 \backslash \mathrm{C}, 0,4.522396,-1.06526,-1.349961 \backslash 0,0,4.519308,-1.923989$ $,-2.19626 \backslash C, 0,4.488799,-1.221364,1.14159 \backslash C, 0,3.833253,-0.903082,2.4907$ $02 \backslash \mathrm{C}, 0,3.451371,0.569845,2.698031 \backslash \mathrm{N}, 0,2.185021,0.828763,2.019565 \backslash \mathrm{C}, 0,1$ $.823446,1.938551,1.388075 \backslash \mathrm{N}, 0,2.447089,3.107896,1.635469 \backslash \mathrm{N}, 0,0.852246$, $1.886287,0.482668 \backslash \mathrm{H}, 0,1.670868,-0.006902,1.699504 \backslash \mathrm{H}, 0,0.490383,0.99203$ $2,0.147423 \backslash \mathrm{H}, 0,0.511123,2.726972,0.033001 \backslash \mathrm{H}, 0,2.122089,3.946059,1.1754$ $82 \backslash 0,0,5.335471,-0.004514,-1.365965 \backslash \mathrm{H}, 0,5.903364,-0.065462,-2.151493 \backslash \mathrm{H}$ $, 0,2.890295,-2.742168,-1.102215 \backslash \mathrm{H}, 0,-4.75879,-1.77683,2.225161 \backslash \mathrm{H}, 0,-4$. $447476,-4.798126,1.71923 \backslash \mathrm{H}, 0,-5.913715,-3.869417,1.402091 \backslash \mathrm{H}, 0,-5.34926$ $6,-4.077475,3.068705 \backslash \mathrm{H}, 0,-3.272707,-1.586211,-1.846572 \backslash \mathrm{H}, 0,-2.882841,0$ $.738791,-2.595526 \backslash \mathrm{H}, 0,-4.399963,0.484044,-1.744395 \backslash \mathrm{H}, 0,-4.534331,1.633$ 
$698,0.899421 \backslash \mathrm{H}, 0,-0.915146,2.207508,-2.732012 \backslash \mathrm{H}, 0,0.539625,4.21188,-2$. $612127 \backslash \mathrm{H}, 0,0.353178,5.802438,-0.722625 \backslash \mathrm{H}, 0,-1.316075,5.446082,1.082936$ $\backslash \mathrm{H}, 0,1.038017,-4.030688,-2.45245 \backslash \mathrm{H}, 0,-0.149226,-4.762102,-1.375505 \backslash \mathrm{H}, 0$ $, 1.523661,-4.576853,-0.831831 \backslash \mathrm{H}, 0,3.09698,-0.09989,-0.112281 \backslash \mathrm{H}, 0,4.852$ $6,-2.254584,1.160228 \backslash \mathrm{H}, 0,5.364194,-0.573555,1.022374 \backslash \mathrm{H}, 0,4.547923,-1.1$ $75932,3.272885 \backslash \mathrm{H}, 0,2.947575,-1.530902,2.651889 \backslash \mathrm{H}, 0,3.351914,0.779438,3$ $.768841 \backslash \mathrm{H}, 0,4.232213,1.225632,2.290713 \backslash \mathrm{H}, 0,2.97566,3.222733,2.486183 \backslash \mathrm{H}$ $, 0,-3.316401,-2.71982,2.587613 \backslash \backslash$ Version=EM64L-G09RevA. 02 $\backslash$ State $=2-A \backslash H F=$ $-1655.4430458 \backslash \mathrm{S} 2=0.755606 \backslash \mathrm{S} 2-1=0 . \backslash \mathrm{S} 2 \mathrm{~A}=0.750026 \backslash \mathrm{RMSD}=6.199 \mathrm{e}-09 \backslash \mathrm{PG}=\mathrm{C} 01$ [ $\mathrm{X}(\mathrm{C} 23 \mathrm{H} 33 \mathrm{~N} 7 \mathrm{O}) \mathrm{S}) \mathrm{\backslash \backslash @}$

Table S11. M06-2X/6-31+G(d,p) Optimized Geometry of AWAR Radical 3b.

\# um062x/6-31+G(d,p) scf=(xqc, tight) pop=none opt cac

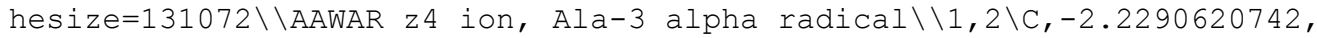
$1.8103366813,-2.0446499977 \backslash C,-1.9629138574,1.8754349506,-0.5575796869 \backslash$ $0,-0.9243367159,2.4019016203,-0.1212699987 \backslash \mathrm{C},-0.9602061628,1.440476782$ $6,-2.8127707208 \backslash \mathrm{N},-2.8830053997,1.3303530387,0.2580457118 \backslash \mathrm{C},-2.8987853$ $425,1.4416709583,1.7146084277 \backslash C,-1.9200190216,0.4510287026,2.383660063$ $3 \backslash 0,-2.2426575362,-0.3572823984,3.2265394865 \backslash \mathrm{C},-4.344019549,1.28809447$ $98,2.2497753496 \backslash C,-5.2427449456,0.3961461423,1.4404104726 \backslash C,-5.3116929$ $367,-1.0477620535,1.4054595941 \backslash C,-6.3211395607,-1.3891970691,0.4734760$ $515 \backslash \mathrm{N},-6.8353507976,-0.2149791797,-0.0310364108 \backslash \mathrm{C},-6.1925576388,0.8476$ $409053,0.5557332163 \backslash \mathrm{C},-4.6439151637,-2.0804488422,2.0909053689 \backslash \mathrm{C},-4.98$ $70717431,-3.3926108943,1.8157418641 \backslash \mathrm{C},-5.9862366947,-3.7080045328,0.87$ $08363371 \backslash \mathrm{C},-6.6686680516,-2.7151558513,0.1898482235 \backslash \mathrm{H},-7.6093780769,-0$ $.1453193705,-0.672256916 \backslash \mathrm{H},-3.6988772845,0.8804209207,-0.1468564014 \backslash \mathrm{N}$, $-0.6175800807,0.6248740318,1.9425096145 \backslash C, 0.5010503542,-0.0781528798,2$ $.3155812903 \backslash \mathrm{C}, 1.6852182293,0.1082802087,1.5061615464 \backslash 0,2.822734783,-0$. $2326388169,1.9209275105 \backslash \mathrm{C}, 0.5210883671,-0.9830165891,3.4966107757 \backslash \mathrm{H},-0$ $.5009209976,1.4264871893,1.3103767021 \backslash \mathrm{N}, 1.5395736994,0.6469982486,0.25$ $4513932 \backslash C, 2.5912064009,1.401762669,-0.4144062043 \backslash C, 2.0926938785,2.8432$ $831698,-0.5698923612 \backslash 0,1.964921351,3.4124023452,-1.6226459328 \backslash \mathrm{C}, 2.9708$ $047537,0.8255885836,-1.780969054 \backslash C, 3.4950879245,-0.6135850349,-1.78075$ $5303 \backslash \mathrm{C}, 4.9026767134,-0.8046013731,-1.2122427106 \backslash \mathrm{N}, 4.8708886693,-0.6920$ $333252,0.2425666827 \backslash \mathrm{C}, 5.912166614,-0.5087352097,1.0331012762 \backslash \mathrm{N}, 7.16542$ $86973,-0.5815255869,0.5583540165 \backslash N, 5.7002249055,-0.2182676134,2.324047$ $9573 \backslash \mathrm{H}, 3.9569430952,-0.6538825174,0.7326013135 \backslash \mathrm{H}, 4.7399089078,-0.13137$ $23273,2.6481896497 \backslash \mathrm{H}, 6.4418913351,-0.281639751,3.0027465925 \backslash \mathrm{H}, 7.958505$ $6701,-0.353633559,1.1369479469 \backslash 0,1.8037157595,3.3835056016,0.620408461$ $9 \backslash \mathrm{H}, 1.3878563144,4.2466027803,0.4646509934 \backslash \mathrm{H}, 0.6038595265,0.8472637763$ $,-0.0810323349 \backslash \mathrm{H},-3.0440549518,1.1121945094,-2.2575738548 \backslash \mathrm{H},-0.1649913$ $193,2.1597360594,-2.5998858478 \backslash \mathrm{H},-1.1510630598,1.4422441333,-3.8876484$ $851 \backslash \mathrm{H},-0.6174164925,0.4372467855,-2.5346704049 \backslash \mathrm{H},-4.2746618627,0.92681$ $55647,3.2794809833 \backslash \mathrm{H},-4.7906857351,2.2859556932,2.2798570643 \backslash \mathrm{H},-6.4737$ $818095,1.8649235023,0.3163766087 \backslash \mathrm{H},-3.8839778727,-1.845069962,2.827165$ $2532 \backslash \mathrm{H},-4.4850953915,-4.197880651,2.3421170414 \backslash \mathrm{H},-6.2305783125,-4.7481$ $677399,0.6812616654 \backslash \mathrm{H},-7.4465732682,-2.9547901154,-0.5283510553 \backslash \mathrm{H},-0.1$ $287041055,-1.8500675266,3.3403959632 \backslash \mathrm{H}, 0.1424644849,-0.4707911451,4.38$ $54907663 \backslash \mathrm{H}, 1.5445494159,-1.3163971766,3.6644363857 \backslash \mathrm{H}, 3.4477716658,1.43$ $39111068,0.2665332132 \backslash \mathrm{H}, 2.0812218794,0.8754839703,-2.4185321911 \backslash \mathrm{H}, 3.70$ $36432736,1.4962055105,-2.2435369354 \backslash \mathrm{H}, 3.5178553431,-0.9655046959,-2.81$ $60474395 \backslash \mathrm{H}, 2.7991903867,-1.2736495754,-1.2466765696 \backslash \mathrm{H}, 5.2823691018,-1$. $7932321291,-1.4978026993 \backslash \mathrm{H}, 5.5766093383,-0.0410134825,-1.6234015629 \backslash \mathrm{H}$, $7.3454163726,-0.9138630335,-0.375825109 \backslash \mathrm{H},-2.5680183735,2.8087165391,-$ $2.3448760134 \backslash \mathrm{H},-2.5261600256,2.4437658474,1.9662968276 \backslash \backslash$ Version $=\mathrm{EM} 64 \mathrm{~L}-$ G09RevA.02 $\backslash \mathrm{HF}=-1655.0060892 \backslash \mathrm{S} 2=0.755838 \backslash \mathrm{S} 2-1=0 . \backslash \mathrm{S} 2 \mathrm{~A}=0.750027 \backslash \mathrm{RMSD}=3.69$ $7 e-10 \backslash \mathrm{RMSF}=5.538 e-06 \backslash \mathrm{Dipole}=-5.4991403,-0.132995,1.3179076 \backslash \mathrm{PG}=\mathrm{C} 01 \quad[\mathrm{X}(\mathrm{C}$ 23H33N7O5) ] \\@ 
Table S12. M06-2X/6-31+G(d,p) Optimized Geometry of AWAR Radical 4a.

\# um062x/6-31+G(d,p) scf=(xqc, tight) pop=none opt cach

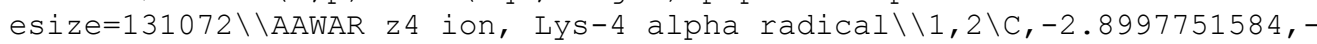
$4.1871690876,2.1787204786 \backslash \mathrm{C},-3.2347443287,-3.3107318346,0.9891555062 \backslash 0$ $,-3.6070517835,-3.7702824231,-0.0854352183 \backslash \mathrm{C},-3.7756634974,-5.43712330$ $41,2.2219033944 \backslash \mathrm{N},-3.0546046746,-1.9658670064,1.1545471412 \backslash \mathrm{C},-3.482608$ $7635,-0.9960421349,0.1524616394 \backslash \mathrm{C},-2.3889200004,-0.7986425522,-0.91104$ $72481 \backslash 0,-1.8304201509,0.2853239033,-1.0838004742 \backslash \mathrm{C},-3.917016484,0.3290$ $283198,0.8083531121 \backslash \mathrm{C},-3.0015884102,0.8855956716,1.8660351592 \backslash \mathrm{C},-2.009$ $2798412,1.9324817729,1.7469486684 \backslash C,-1.4650472082,2.1409377505,3.04137$ $55266 \backslash \mathrm{N},-2.0873476969,1.2653351971,3.8961900743 \backslash \mathrm{C},-3.0210092634,0.5390$ $982138,3.1967502604 \backslash \mathrm{C},-1.5692986398,2.7493882574,0.686390454 \backslash \mathrm{C},-0.6302$ $074548,3.7339648639,0.9504559898 \backslash \mathrm{C},-0.0874064633,3.9107679521,2.242795$ $4691 \backslash \mathrm{C},-0.4899571609,3.1146689245,3.3041222991 \backslash \mathrm{H},-1.9510633735,1.22682$ $24113,4.8947880685 \backslash \mathrm{H},-2.7791057091,-1.6087925685,2.0610428234 \backslash \mathrm{N},-2.075$ $2489049,-1.9116376059,-1.6067655317 \backslash C,-1.1068648498,-1.9194772394,-2.7$ $02798137 \backslash \mathrm{C}, 0.296302118,-1.6280061538,-2.1493073723 \backslash 0,0.9674855963,-2.5$ $178983857,-1.639071448 \backslash \mathrm{C},-1.5376242583,-1.0481202921,-3.8814605349 \backslash \mathrm{H},-$ $2.6085689174,-2.7583568036,-1.4060863855 \backslash N, 0.7363115263,-0.3303655305$, $-2.2379179467 \backslash C, 1.9977021953,0.1134160919,-1.9014867046 \backslash C, 2.0832079314$ $, 1.4590456665,-1.3748880084 \backslash 0,3.098864824,1.9460278593,-0.8950496784 \backslash \mathrm{C}$ $, 3.2557076133,-0.665587558,-2.1400962137 \backslash \mathrm{C}, 3.8256690869,-1.5296008438$, $-0.9853840104 \backslash \mathrm{C}, 3.5332527781,-0.9907326048,0.4124504069 \backslash \mathrm{N}, 2.1042125827$ $,-1.1854630247,0.684601727 \backslash \mathrm{C}, 1.2303416326,-0.2990564146,1.145631936 \backslash \mathrm{N}$, $1.5938636137,0.9137345325,1.5668295392 \backslash \mathrm{N},-0.0655475675,-0.6519816977,1$ $.2405612946 \backslash \mathrm{H}, 1.6995394827,-1.9788846564,0.1892560454 \backslash \mathrm{H},-0.3505644653$, $-1.5459727128,0.8591150896 \backslash \mathrm{H},-0.758608606,0.0903933568,1.1759188325 \backslash \mathrm{H}$, $0.8925826646,1.5577035948,1.9188576258 \backslash 0,0.9022630879,2.112018683,-1.3$ $941183249 \backslash \mathrm{H}, 1.0166316597,2.9652677943,-0.9482984487 \backslash \mathrm{H},-0.0045902845,0$. $3719727704,-2.2672476526 \backslash \mathrm{H},-2.9788560849,-3.6120478666,3.1076111099 \backslash \mathrm{H}$, $-3.6981529624,-5.9843572477,1.2813145145 \backslash \mathrm{H},-4.8252524628,-5.1740764285$ $, 2.3740656792 \backslash \mathrm{H},-3.4627477895,-6.0901117399,3.0386881155 \backslash \mathrm{H},-4.34854864$ $01,-1.4319894715,-0.3612074598 \backslash \mathrm{H},-4.0509987073,1.0663270379,0.01247891$ $17 \backslash \mathrm{H},-4.9009143762,0.1537585388,1.2542469111 \backslash \mathrm{H},-3.6751059031,-0.150516$ $5549,3.7160504034 \backslash \mathrm{H},-1.9622931712,2.6012254358,-0.3135802727 \backslash \mathrm{H},-0.3288$ $082525,4.4147425808,0.1582823438 \backslash \mathrm{H}, 0.642857444,4.6955249426,2.41221011$ $16 \backslash \mathrm{H},-0.0906284031,3.2606284145,4.3028836437 \backslash \mathrm{H},-1.0381353094,-2.960943$ $7867,-3.026047264 \backslash \mathrm{H},-1.7060249351,-0.0110666623,-3.5840002919 \backslash \mathrm{H},-2.471$ $9794537,-1.4395162216,-4.289570705 \backslash \mathrm{H},-0.7809730281,-1.0735056479,-4.66$ $97646778 \backslash \mathrm{H}, 3.1058843773,-1.3072501098,-3.013185089 \backslash \mathrm{H}, 4.0092695594,0.08$ $38762479,-2.4012549859 \backslash \mathrm{H}, 4.9094431854,-1.5953852362,-1.1102729429 \backslash \mathrm{H}, 3$. $4330064013,-2.5482423382,-1.0594307727 \backslash \mathrm{H}, 4.1149550117,-1.5313923321,1$. $1643508678 \backslash \mathrm{H}, 3.7930727662,0.0690815816,0.4677170395 \backslash \mathrm{H}, 2.4846747437,1.3$ $090687633,1.2953885762 \backslash \mathrm{H},-1.8457414869,-4.4739690518,2.0698800107 \backslash \backslash$ Ver

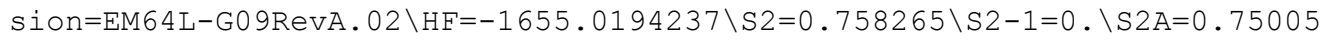
$2 \backslash \mathrm{RMSD}=1.020 \mathrm{e}-09 \backslash \mathrm{RMSF}=8.385 \mathrm{e}-06 \backslash \mathrm{Dipole}=1.3363867,-0.4803452,1.093262 \backslash \mathrm{P}$ $\mathrm{G}=\mathrm{C} 01 \quad[\mathrm{X}(\mathrm{C} 23 \mathrm{H} 33 \mathrm{~N} 705)] \backslash \backslash \mathrm{Q}$

\section{Table S13. M06-2X/6-31+G(d,p) Optimized Geometry of AWAR Radical 5a.}

\# um062x/6-31+G(d,p) scf=(xqc, tight) pop=none opt cac

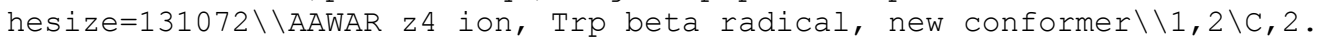
$709229194,-2.3570237573,2.9282535981 \backslash C, 2.9153783879,-2.4979572424,1.43$ $42724236 \backslash 0,4.0242913944,-2.6809987377,0.9387165307 \backslash \mathrm{C}, 3.8565333024,-1.5$ $910358313,3.5824980045 \backslash \mathrm{N}, 1.7948736844,-2.4444360276,0.6633762039 \backslash \mathrm{C}, 1.8$ $244722281,-2.6625917532,-0.7804097864 \backslash \mathrm{C}, 2.2457819437,-1.3375387652,-1$. $4575724306 \backslash 0,1.431253955,-0.6278444632,-2.0626357836 \backslash \mathrm{C}, 0.4841877492,-3$ $.0816879846,-1.2976185119 \backslash C,-0.4945878348,-3.7951029817,-0.5848009654 \backslash$ $\mathrm{C},-1.8960976541,-3.8820398601,-0.9867700879 \backslash \mathrm{C},-2.5707428372,-4.6527349$ $299,-0.0186722383 \backslash \mathrm{N},-1.6398566272,-5.0327803282,0.9298473285 \backslash \mathrm{C},-0.4082$ $293773,-4.5267345268,0.6070087448 \backslash C,-2.6167844019,-3.3467877598,-2.063$ $1867756 \backslash \mathrm{C},-3.980731045,-3.5966295483,-2.1447300848 \backslash \mathrm{C},-4.6329779057,-4$. $3732938351,-1.1683877562 \backslash \mathrm{C},-3.9411540468,-4.9121206461,-0.0928234123 \backslash \mathrm{H}$ 
$,-1.8273207112,-5.6309235878,1.7195545059 \backslash \mathrm{H}, 0.9036132534,-2.2164342201$ $, 1.0829539292 \backslash \mathrm{N}, 3.5222868862,-0.9766329639,-1.2714040352 \backslash \mathrm{C}, 3.993013496$ $3,0.3595568434,-1.6178384698 \backslash C, 2.8951388802,1.3592996201,-1.2049260509$ $\backslash 0,2.3123521431,1.2122452939,-0.1273627587 \backslash \mathrm{C}, 4.4363346336,0.4343963735$ $,-3.0777561326 \backslash \mathrm{H}, 4.0858031508,-1.5648645581,-0.6581145257 \backslash \mathrm{N}, 2.62790688$ $21,2.3632191364,-2.054777577 \backslash \mathrm{C}, 1.3999339244,3.1450422697,-2.0164140428$ $\backslash C, 1.2939296139,3.8044318734,-3.3881533101 \backslash 0,2.1304732569,3.7172543156$ $,-4.2516488171 \backslash \mathrm{C}, 1.2928793439,4.2056624846,-0.8979209918 \backslash \mathrm{C}, 0.783810979$ $9,3.7482378598,0.4783510199 \backslash \mathrm{C},-0.5661330698,3.0215958196,0.4677048845 \backslash$ $\mathrm{N},-0.3930268512,1.6191215465,0.0804977286 \backslash \mathrm{C},-1.3787608795,0.7737925949$ $,-0.1849450652 \backslash \mathrm{N},-2.6502544851,1.1086277497,0.0945021774 \backslash \mathrm{N},-1.10643015$ $61,-0.4100423792,-0.7363733125 \backslash \mathrm{H}, 0.5806734972,1.2873980478,-0.00000268$ $32 \backslash \mathrm{H},-0.2128129859,-0.5613362397,-1.2222700037 \backslash \mathrm{H},-1.8104689374,-1.1349$ $926697,-0.8036295293 \backslash \mathrm{H},-3.4135677347,0.5212909711,-0.2036390275 \backslash 0,0.14$ $39186499,4.4714776347,-3.529627697 \backslash \mathrm{H}, 0.1232982934,4.8597205627,-4.4194$ $406926 \backslash \mathrm{H}, 3.0971919014,2.382665298,-2.9539025949 \backslash \mathrm{H}, 1.7439322464,-1.8817$ $686676,3.1336922908 \backslash \mathrm{H}, 4.8071608875,-2.0781451864,3.3596456579 \backslash \mathrm{H}, 3.7233$ $516127,-1.5565132916,4.665562369 \backslash \mathrm{H}, 3.9025797871,-0.5649152984,3.207410$ $5557 \backslash \mathrm{H}, 2.6095451304,-3.4111153049,-0.9692806086 \backslash \mathrm{H}, 0.2409744569,-2.7405$ $104522,-2.2975846953 \backslash \mathrm{H}, 0.4568530405,-4.7511803738,1.2137958332 \backslash \mathrm{H},-2.11$ $62255887,-2.7704117163,-2.8381782928 \backslash \mathrm{H},-4.5513157817,-3.2054759546,-2$. $9806470231 \backslash \mathrm{H},-5.6973334025,-4.5617680777,-1.2626263044 \backslash \mathrm{H},-4.4469136937$ $,-5.5138618577,0.6556362752 \backslash \mathrm{H}, 4.8464062997,0.5634984209,-0.9619038873 \backslash$ $\mathrm{H}, 3.5893232225,0.2603227551,-3.7476788305 \backslash \mathrm{H}, 5.1846827054,-0.3380089757$ $,-3.2634952779 \backslash \mathrm{H}, 4.8984612668,1.3984345408,-3.3106477969 \backslash \mathrm{H}, 0.542964826$ $8,2.4597958196,-1.9632430617 \backslash \mathrm{H}, 2.2769731953,4.6708689498,-0.7799604138$ $\backslash \mathrm{H}, 0.6116078739,4.9821899587,-1.2599679798 \backslash \mathrm{H}, 0.6548187409,4.6507416924$ $, 1.0835306218 \backslash \mathrm{H}, 1.5223204729,3.1252242923,0.9874352224 \backslash \mathrm{H},-1.0027239863$ $, 3.071843,1.4732863008 \backslash \mathrm{H},-1.2588846251,3.5079668639,-0.2334388173 \backslash \mathrm{H},-2$ $.8640538938,1.9018431827,0.6779741816 \backslash \mathrm{H}, 2.6581411214,-3.3753470178,3.3$

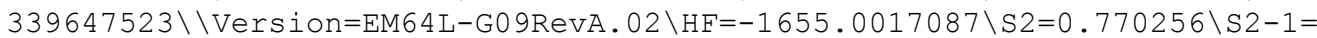
$0 . \backslash \mathrm{S} 2 \mathrm{~A}=0.750144 \backslash \mathrm{RMSD}=6.363 e-09 \backslash \mathrm{RMSF}=5.302 \mathrm{e}-06 \backslash \mathrm{Dipole}=2.2584683,2.40872$ $38,1.3047763 \backslash \mathrm{PG}=\mathrm{C} 01 \quad[\mathrm{X}(\mathrm{C} 23 \mathrm{H} 33 \mathrm{~N} 705)] \backslash \backslash \mathrm{e}$

Table S14. M06-2X/6-31+G(d,p) Optimized Geometry of AWAR Radical 5b.

\# um062x/6-31+G(d,p) scf=(xqc, tight) pop=none opt cac

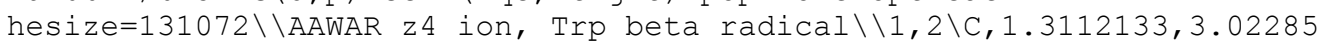
$72373,3.1377792598 \backslash \mathrm{C}, 1.2886720549,1.5233765329,3.351395969 \backslash 0,2.2238914$ $52,0.9286599205,3.8879161276 \backslash \mathrm{C}, 2.6997630421,3.5133018842,2.7360415565 \backslash$ $\mathrm{N}, 0.1766060611,0.8672316536,2.9297239048 \backslash \mathrm{C},-0.1076578471,-0.5200834654$ $, 3.3350801442 \backslash \mathrm{C}, 0.6351866726,-1.4863890942,2.3794482172 \backslash 0,0.0512804131$ $,-2.1463796254,1.5176111123 \backslash C,-1.5830992155,-0.7546939317,3.4107004377$ $\backslash C,-2.4756916235,-0.4879348033,2.3639919437 \backslash C,-3.9339853172,-0.4972508$ $532,2.4575475547 \backslash \mathrm{C},-4.4417826991,-0.1648013337,1.1877853321 \backslash \mathrm{N},-3.36087$ $89085,0.0442726202,0.3458236699 \backslash \mathrm{C},-2.192458618,-0.1496834163,1.0322567$ $598 \backslash C,-4.8264331562,-0.7495334625,3.5049530554 \backslash C,-6.1895445646,-0.6695$ $342364,3.2557917726 \backslash \mathrm{C},-6.6742331726,-0.3365641338,1.9775870029 \backslash \mathrm{C},-5.80$ $9605427,-0.0767617466,0.9242172854 \backslash \mathrm{H},-3.4294070412,0.2557831655,-0.636$ $6945335 \backslash \mathrm{H},-0.616597239,1.4027522576,2.6001329146 \backslash \mathrm{N}, 1.9715422466,-1.527$ $2197285,2.5718340925 \backslash \mathrm{C}, 2.9040779565,-2.4391415361,1.9189129438 \backslash \mathrm{C}, 3.137$ $1999919,-2.1406145359,0.4374076147 \backslash 0,4.2995858879,-2.0929916304,-0.011$ $6814471 \backslash \mathrm{C}, 2.5292284674,-3.9156652914,2.1042222033 \backslash \mathrm{H}, 2.3508585992,-0.83$ $00157653,3.2159876471 \backslash \mathrm{N}, 2.0513299591,-2.0093152214,-0.3326522352 \backslash \mathrm{C}, 2.0$ $937272872,-1.5757955118,-1.7133923832 \backslash \mathrm{C}, 1.2810123488,-0.2981332506,-1$. $9270038298 \backslash 0,0.9419248801,0.0915294617,-3.0141097165 \backslash \mathrm{C}, 1.61936329,-2.6$ $575406179,-2.6984221974 \backslash \mathrm{C}, 2.4505984959,-3.944199197,-2.7240091841 \backslash \mathrm{C}, 3$. $8272770254,-3.8274649725,-3.3837079406 \backslash N, 4.7528009566,-3.1392960659,-2$ $.4873111773 \backslash \mathrm{C}, 5.9167962582,-2.6063661375,-2.815383389 \backslash \mathrm{N}, 6.4542627612,-$ $2.8083980496,-4.0265275417 \backslash \mathrm{N}, 6.545273532,-1.8323398062,-1.9204774298 \backslash \mathrm{H}$ , $4.4612184233,-2.9463152651,-1.5179029731 \backslash \mathrm{H}, 6.097582807,-1.6654706185$, $-1.0232612007 \backslash \mathrm{H}, 7.5150814146,-1.5830183175,-2.0335075611 \backslash \mathrm{H}, 7.292134102$ $8,-2.3275580884,-4.3146594349 \backslash 0,1.0213871629,0.3646390781,-0.7885446 \backslash \mathrm{H}$ 
$, 0.5745916438,1.1913462667,-1.0336725469 \backslash \mathrm{H}, 1.1426529094,-2.1061002813$, $0.1456601569 \backslash \mathrm{H}, 0.5564731156,3.3123349821,2.3990319341 \backslash \mathrm{H}, 3.4361241531,3$ $.2043640222,3.4798558381 \backslash \mathrm{H}, 2.7129721627,4.6022511208,2.6572996134 \backslash \mathrm{H}, 2$. $9972217206,3.0948641722,1.7699727284 \backslash \mathrm{H}, 0.3346255644,-0.6558585576,4.32$ $85217715 \backslash \mathrm{H},-1.9822399087,-1.0583799677,4.3709516555 \backslash \mathrm{H},-1.2339742843,-0$ $.1070442524,0.5342087461 \backslash \mathrm{H},-4.4637917135,-1.004039525,4.4965085073 \backslash \mathrm{H},-$ $6.8952419821,-0.8648855002,4.0561810924 \backslash \mathrm{H},-7.7450972085,-0.2800174897$, $1.81208085 \backslash \mathrm{H},-6.1825047189,0.1826578523,-0.0616414321 \backslash \mathrm{H}, 3.8746444911,-$ $2.2613850429,2.386303169 \backslash \mathrm{H}, 1.557152565,-4.1362523199,1.659930494 \backslash \mathrm{H}, 2.4$ $873873472,-4.1451882946,3.1709346386 \backslash \mathrm{H}, 3.2917678702,-4.5502387107,1.64$ $34753537 \backslash \mathrm{H}, 3.1267478113,-1.2804701635,-1.931914693 \backslash \mathrm{H}, 0.588796712,-2.91$ $92139658,-2.4320025066 \backslash \mathrm{H}, 1.579304318,-2.2103691377,-3.6965970154 \backslash \mathrm{H}, 1.8$ $888473836,-4.6956154151,-3.2858236508 \backslash \mathrm{H}, 2.5679182078,-4.3497806232,-1$. $7099395064 \backslash \mathrm{H}, 4.2119005003,-4.8290507772,-3.6102229065 \backslash \mathrm{H}, 3.7428623479,-$ $3.2646802762,-4.3228188093 \backslash \mathrm{H}, 6.0498520972,-3.4719603155,-4.6684248271 \backslash$ $\mathrm{H}, 1.0142588737,3.4763740983,4.0911275983 \backslash \backslash$ Version=EM6 4L-G0 9RevA.02 $\backslash \mathrm{HF}=$ $-1654.9911615 \backslash \mathrm{S} 2=0.770799 \backslash \mathrm{S} 2-1=0 . \backslash \mathrm{S} 2 \mathrm{~A}=0.750146 \backslash \mathrm{RMSD}=6.965 e-09 \backslash \mathrm{RMSF}=3.2$ $46 e-06 \backslash \mathrm{Dipole}=3.560784,1.7952841,-6.0745985 \backslash \mathrm{PG}=\mathrm{C} 01 \quad[\mathrm{X}(\mathrm{C} 23 \mathrm{H} 33 \mathrm{~N} 705)] \backslash \backslash @$

\section{Table S15. M06-2X/6-31+G(d,p) Optimized Geometry of AFAR Radical 6a.}

\# um062x/6-31+G(d,p) scf=(xqc, tight) pop=none cachesize=

131072 opt freq $\backslash \backslash A A F A R$ z4 ion, new conformer, radical close to Arg $\backslash \backslash 1$, $2 \backslash \mathrm{H}, 1.4029276568,1.8792484159,0.6663293728 \backslash \mathrm{N}, 0.9637505558,1.3606685686$ $,-0.0825383859 \backslash \mathrm{C}, 1.76671189,0.3729307712,-0.776900624 \backslash \mathrm{C}, 1.3744173347,-$ $1.0253176732,-0.2772232461 \backslash 0,1.2406723061,-1.231935409,0.9236082798 \backslash \mathrm{C}$, $3.2584587006,0.6216754186,-0.5065735261 \backslash C, 4.1112915041,-0.4175065434,-$ $1.1932888191 \backslash \mathrm{C}, 4.3802870393,-0.3183385694,-2.5615163735 \backslash \mathrm{C}, 4.5793188018$ $,-1.5292218188,-0.4889357078 \backslash \mathrm{C}, 5.3040473437,-2.5256413855,-1.140361260$ $7 \backslash \mathrm{C}, 5.1017262103,-1.3144878605,-3.2163190954 \backslash \mathrm{H}, 4.3723177205,-1.6140778$ $751,0.575567524 \backslash \mathrm{N}, 1.2161102643,-1.9759859504,-1.2313129425 \backslash \mathrm{C}, 0.5595994$ $994,-3.2586556866,-0.9533044263 \backslash \mathrm{C},-0.8496834126,-2.8662452411,-0.48888$ $30705 \backslash C, 1.3795416657,-4.1536694561,-0.0314699807 \backslash 0,-1.6792513161,-2.51$ $35203577,-1.3321461915 \backslash \mathrm{C},-0.3953516374,1.270161039,-0.0922961225 \backslash \mathrm{C},-1$. $119704249,2.1825134337,0.7691682052 \backslash 0,-0.9571192838,0.4291485774,-0.82$ $70655273 \backslash \mathrm{C},-2.599035644,2.1623513775,0.8667181631 \backslash \mathrm{N},-1.0744805609,-2.8$ $146148492,0.8388059439 \backslash \mathrm{C},-2.0005441596,-1.8269676573,1.3927999083 \backslash \mathrm{C},-1$ $.323913385,-1.1046368026,2.5483955829 \backslash 0,-1.4693306784,0.0692080755,2.7$ $823987163 \backslash 0,-0.5993805832,-1.9339667441,3.3104704268 \backslash \mathrm{C},-3.3300291711,-$ $2.4316028658,1.8768765062 \backslash \mathrm{C},-4.1816950404,-3.0778948272,0.7776016284 \backslash \mathrm{C}$ $,-4.9691258993,-2.1000688583,-0.0971789367 \backslash N,-4.0660914536,-1.30733256$ $63,-0.9279370242 \backslash \mathrm{C},-4.4069747293,-0.2110065715,-1.5840233713 \backslash \mathrm{N},-3.4542$ $642174,0.5318015269,-2.1533512522 \backslash N,-5.6963301072,0.1678510048,-1.6561$ $749465 \backslash \mathrm{H}, 1.5669473506,0.4512025221,-1.8523696635 \backslash \mathrm{H}, 3.4212590471,0.5850$ $352173,0.5770551089 \backslash \mathrm{H}, 3.5104309384,1.6279472102,-0.8589014346 \backslash \mathrm{H}, 4.0338$ $455106,0.5516828093,-3.1160848762 \backslash \mathrm{H}, 1.1962070784,-1.6605486124,-2.1909$ $233165 \backslash \mathrm{H}, 0.4256905108,-3.7494028541,-1.9195889127 \backslash \mathrm{H}, 0.8414747947,-5.08$ $27731221,0.1756246968 \backslash \mathrm{H}, 2.3197232733,-4.4012389362,-0.5294139667 \backslash \mathrm{H}, 1.6$ $255921776,-3.6542159502,0.9079382784 \backslash \mathrm{H},-0.5534830654,2.8978476118,1.35$ $69900892 \backslash \mathrm{H},-3.0468675118,2.942987674,0.2353457928 \backslash \mathrm{H},-2.9941402747,1.19$ $4379571,0.5470129103 \backslash \mathrm{H},-2.9142809645,2.3471781379,1.8962242733 \backslash \mathrm{H},-0.27$ $1551707,-3.010612405,1.4264389376 \backslash \mathrm{H},-2.1649971001,-1.0560364946,0.6362$ $628469 \backslash \mathrm{H},-0.1649272002,-1.4180834814,4.0081473927 \backslash \mathrm{H},-3.9038261358,-1.6$ $408682889,2.3774869045 \backslash \mathrm{H},-3.0945947806,-3.1887410452,2.6324961448 \backslash \mathrm{H},-3$ $.5582394255,-3.7181498012,0.1420916047 \backslash \mathrm{H},-4.916312769,-3.734544444,1.2$ $52950138 \backslash \mathrm{H},-5.5559305071,-1.4228898248,0.5364769131 \backslash \mathrm{H},-5.6631070712,-2$ $.6682701644,-0.7311089538 \backslash \mathrm{H},-2.4889323309,0.464888422,-1.8028105858 \backslash \mathrm{H}$, $-3.6960677145,1.2811149641,-2.7819905881 \backslash \mathrm{H},-6.4372557479,-0.4885836327$ $,-1.4656136388 \backslash \mathrm{H},-5.9522485218,1.0592534353,-2.0502992657 \backslash \mathrm{H},-3.1227259$ $563,-1.7085884369,-1.1117548937 \backslash \mathrm{C}, 5.5638179386,-2.421904633,-2.5059246$ $09 \backslash \mathrm{H}, 5.3125910184,-1.2214622057,-4.2769763017 \backslash \mathrm{H}, 6.1330459548,-3.194532$ $4634,-3.0126464986 \backslash \mathrm{H}, 5.6729672267,-3.3792234185,-0.5801239535 \backslash \backslash$ Version $=\mathrm{EM} 64 \mathrm{~L}-\mathrm{G} 0$ 9RevA. 02 $\backslash \mathrm{HF}=-1523.4679681 \backslash \mathrm{S} 2=0.75642 \backslash \mathrm{S} 2-1=0 . \backslash \mathrm{S} 2 \mathrm{~A}=0.750034 \backslash \mathrm{RMS}$ 
$\mathrm{D}=4.055 e-09 \backslash \mathrm{RMSF}=7.831 e-06 \backslash \mathrm{Dipole}=-3.8544768,0.999119,-0.3603812 \backslash \mathrm{PG}=\mathrm{C} 0$

$1[\mathrm{X}(\mathrm{C} 21 \mathrm{H} 32 \mathrm{~N} 6 \mathrm{O} 5)] \backslash \backslash \mathrm{Q}$

Table S16. M06-2X/6-31+G(d,p) Optimized Geometry of AFAR Radical 7.

\# um062x/6-31+G(d,p) scf=(xqc, tight) pop=none opt cac

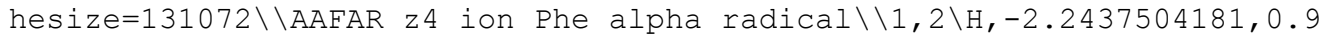
$78751463,-2.8465127041 \backslash N,-1.6846908749,0.6288021231,-2.0773243595 \backslash \mathrm{C},-2$ $.3140987745,-0.0902378378,-1.0751394821 \backslash C,-1.601644751,-1.2644893086,-$ $0.5526202548 \backslash 0,-0.7651997968,-1.8737841247,-1.2180488398 \backslash \mathrm{C},-3.70608797$ $01,0.2731994774,-0.6664638342 \backslash C,-3.7951716516,0.9280492448,0.709647508$ $3 \backslash \mathrm{C},-2.7319079577,1.665737026,1.2391993716 \backslash \mathrm{C},-4.9702018739,0.796194172$ $2,1.4550574569 \backslash C,-5.0900791234,1.4047478846,2.7020677339 \backslash C,-2.85100809$ $53,2.2717014696,2.4903119341 \backslash \mathrm{H},-5.7973786351,0.2155369285,1.0540559865$ $\backslash \mathrm{N},-1.9670778484,-1.6793257281,0.7025065195 \backslash \mathrm{C},-1.2386519707,-2.7862498$ $934,1.3046601415 \backslash \mathrm{C}, 0.2646216033,-2.5346289842,1.0905348143 \backslash \mathrm{C},-1.756207$ $922,-4.132711103,0.8044042151 \backslash 0,0.7369304023,-1.4356545904,1.391229622$ $\backslash \mathrm{C},-0.3419372993,0.9335006121,-2.0730892772 \backslash \mathrm{C}, 0.2002560317,1.485263136$ $1,-3.3729213317 \backslash 0,0.3384848472,0.7588524029,-1.0712376931 \backslash \mathrm{C}, 1.56397263$ $9,2.1410184936,-3.2020063315 \backslash N, 1.0123158738,-3.547567697,0.6076227826 \backslash$ C, $2.3151622589,-3.2875914358,0.0146870968 \backslash C, 2.488707237,-4.0365368425$, $-1.2993922359 \backslash 0,3.3933796813,-3.812083255,-2.0594094055 \backslash 0,1.5665300912$ $,-4.9924150038,-1.4970959515 \backslash \mathrm{C}, 3.4874585161,-3.6652373462,0.9409911322$ $\backslash C, 3.5685300197,-2.8726575636,2.2502026442 \backslash C, 4.1940084535,-1.483338766$ $5,2.1212500637 \backslash \mathrm{N}, 3.3169250533,-0.5755570656,1.3846921761 \backslash \mathrm{C}, 3.714529136$ $5,0.5520683877,0.8203759431 \backslash \mathrm{N}, 2.8885200306,1.2436091603,0.0335287484 \backslash \mathrm{N}$ $, 4.9672512304,1.004876305,1.0161205277 \backslash \mathrm{H},-4.3359771745,-0.6244396463,-$ $0.6647789055 \backslash \mathrm{H},-4.1305988812,0.9572103655,-1.4120907279 \backslash \mathrm{H},-1.802389807$ $6,1.7561286686,0.6802551499 \backslash \mathrm{H},-2.3878527684,-0.9966885322,1.3205305507$ $\backslash \mathrm{H},-1.395800084,-2.7133207247,2.385633027 \backslash \mathrm{H},-1.2597854482,-4.966261971$ $2,1.3109324218 \backslash \mathrm{H},-2.8237152854,-4.2046039135,1.019268962 \backslash \mathrm{H},-1.61904779$ $65,-4.2201267367,-0.2774083623 \backslash \mathrm{H}, 0.2620241322,0.6438425853,-4.07393549$ $43 \backslash \mathrm{H}, 1.9210551615,2.5294505303,-4.1575961871 \backslash \mathrm{H}, 1.5076005954,2.97401985$ $7,-2.4947690674 \backslash \mathrm{H}, 2.2933997958,1.4165301589,-2.8316390783 \backslash \mathrm{H}, 0.51832568$ $6,-4.352203024,0.2438609276 \backslash \mathrm{H}, 2.3597437509,-2.2284342203,-0.256117585 \backslash$ $\mathrm{H}, 1.7530369367,-5.4274271796,-2.3446401572 \backslash \mathrm{H}, 4.4180004693,-3.552524138$ $2,0.372989828 \backslash \mathrm{H}, 3.3827257774,-4.7293531661,1.1811905205 \backslash \mathrm{H}, 2.5795797616$ $,-2.793406712,2.7164477062 \backslash \mathrm{H}, 4.1959984334,-3.4310933195,2.9511769849 \backslash \mathrm{H}$ $, 5.1484969765,-1.5680416841,1.586267137 \backslash \mathrm{H}, 4.3915371588,-1.0789416059,3$ $.1225711666 \backslash \mathrm{H}, 1.9718059683,0.8931562476,-0.262426951 \backslash \mathrm{H}, 3.1262817243,2$. $1818033894,-0.2472981 \backslash \mathrm{H}, 5.5612078642,0.6038495388,1.7235153584 \backslash \mathrm{H}, 5.330$ $3373196,1.7761384185,0.479300508 \backslash \mathrm{H}, 2.3075308605,-0.8184399594,1.358747$ $0684 \backslash \mathrm{C},-4.0296437512,2.1456494338,3.2224720402 \backslash \mathrm{H},-2.0199363026,2.84228$ $46555,2.8929009747 \backslash \mathrm{H},-4.1217989916,2.6190183836,4.1945134105 \backslash \mathrm{H},-6.0100$ $813761,1.2974601476,3.2677700471 \backslash \mathrm{H},-0.5245748483,2.1899054128,-3.79736$

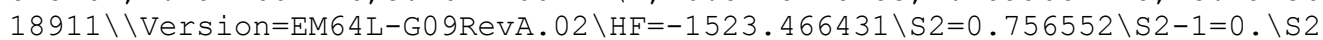
$\mathrm{A}=0.750035 \backslash \mathrm{RMSD}=4.373 \mathrm{e}-09 \backslash \mathrm{RMSF}=4.913 \mathrm{e}-06 \backslash \mathrm{Dipole}=-1.5759211,1.9375192,-$ $0.4998442 \backslash \mathrm{PG}=\mathrm{C} 01 \quad[\mathrm{X}(\mathrm{C} 21 \mathrm{H} 32 \mathrm{~N} 605)] \backslash \backslash @$

\section{Table S17. M06-2X/6-31+G(d,p) Optimized Geometry of AFAR Radical 8a.}

\# um062x/6-31+G(d,p) scf=(xqc, tight) pop=none opt cach

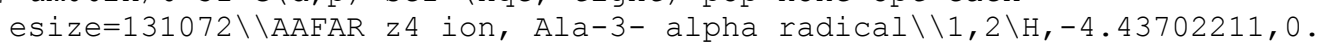
$568192368,-1.6837188484 \backslash \mathrm{N},-3.617687478,0.513144425,-1.0935741513 \backslash \mathrm{C},-3$. $6500287092,-0.4032047668,0.0428442987 \backslash \mathrm{C},-2.8520821438,-1.6670311501,-0$ $.3224233076 \backslash 0,-3.3377302853,-2.6091775459,-0.9115083973 \backslash \mathrm{C},-5.088393492$ $3,-0.7659614519,0.4103651358 \backslash C,-5.1355078929,-1.5364737899,1.710419140$ $3 \backslash C,-5.1255482513,-0.8481302495,2.9278380688 \backslash C,-5.1561644076,-2.933709$ $3228,1.7214693611 \backslash \mathrm{C},-5.1675124104,-3.6288290614,2.9303005483 \backslash \mathrm{C},-5.1359$ $453024,-1.5408294171,4.1360413306 \backslash \mathrm{H},-5.1636152243,-3.4739630162,0.7785$ $840081 \backslash \mathrm{N},-1.5154664947,-1.5754289434,0.0191462119 \backslash \mathrm{C},-0.5176715793,-2.4$ $732046276,-0.2727636828 \backslash \mathrm{C}, 0.8186882544,-1.9271116663,-0.3580209181 \backslash \mathrm{C},-$ $0.7896886095,-3.9231538605,-0.4520709685 \backslash 0,1.840643493,-2.64674759,-0$. $2336104215 \backslash C,-2.5268341513,1.2488127144,-1.4065316314 \backslash C,-2.640813144,2$ 
$.1703081665,-2.6016008895 \backslash 0,-1.4835227256,1.1648542217,-0.7462863456 \backslash \mathrm{C}$ $,-2.0333670055,3.5418574181,-2.306377325 \backslash N, 0.9351221464,-0.5803310828$, $-0.5875083248 \backslash \mathrm{C}, 2.013678577,0.2115850721,-0.0294865698 \backslash \mathrm{C}, 1.4949445804$, $1.1000527519,1.1056830123 \backslash 0,2.0136432885,2.1298806935,1.445377451 \backslash 0,0$. $4158805311,0.5747928373,1.715818469 \backslash \mathrm{C}, 2.7361869019,1.0694289873,-1.075$ $3779882 \backslash \mathrm{C}, 3.4038201311,0.2903449448,-2.2125110852 \backslash \mathrm{C}, 4.6435866219,-0.51$ $72416696,-1.8217251642 \backslash \mathrm{N}, 4.2537853852,-1.7302988684,-1.1062973435 \backslash \mathrm{C}, 5$. $0621250766,-2.5049311398,-0.4030619871 \backslash \mathrm{N}, 4.5346746354,-3.4604735387,0$. $3720916634 \backslash \mathrm{N}, 6.3916712517,-2.3279435949,-0.4410171359 \backslash \mathrm{H},-3.1672902982$, $0.1042687381,0.8863344408 \backslash \mathrm{H},-5.5191056856,-1.3604270625,-0.4026162287 \backslash$ $\mathrm{H},-5.6606402461,0.1634926936,0.5044372294 \backslash \mathrm{H},-5.1267970667,0.240257645$, $2.9294324734 \backslash \mathrm{H},-1.2355741392,-0.675181686,0.4042606516 \backslash \mathrm{H}, 0.1563756639$, $-4.4644729405,-0.4295126055 \backslash \mathrm{H},-1.4501149651,-4.2889484045,0.3395760189$ $\backslash \mathrm{H},-1.3011433841,-4.1157428356,-1.4010423184 \backslash \mathrm{H},-3.6845453336,2.2625325$ $589,-2.9165548407 \backslash \mathrm{H},-2.5861437956,4.0516666045,-1.5132158855 \backslash \mathrm{H},-0.9960$ $255257,3.4363528449,-1.9834195035 \backslash \mathrm{H},-2.0623269686,4.1684201738,-3.1997$ $858248 \backslash \mathrm{H}, 0.098507744,-0.0526503192,-0.8327335598 \backslash \mathrm{H}, 2.7122443092,-0.482$ $9212054,0.4526893204 \backslash \mathrm{H}, 0.1625253873,1.1822672789,2.4296217287 \backslash \mathrm{H}, 3.4686$ $736949,1.6964793866,-0.5575954794 \backslash \mathrm{H}, 1.9985617111,1.754577193,-1.509804$ $1832 \backslash \mathrm{H}, 2.679140147,-0.3773827911,-2.6955081367 \backslash \mathrm{H}, 3.7168083651,1.008450$ $2928,-2.9756944135 \backslash \mathrm{H}, 5.2960316295,0.0889194994,-1.1789354722 \backslash \mathrm{H}, 5.20142$ $55219,-0.7889899569,-2.7262602889 \backslash \mathrm{H}, 3.5216291997,-3.5382918651,0.42648$ $94696 \backslash \mathrm{H}, 5.1064361081,-4.1910200155,0.7647861968 \backslash \mathrm{H}, 6.8103750524,-1.6926$ $270477,-1.1018096902 \backslash \mathrm{H}, 7.0067018288,-2.8550859391,0.1586298424 \backslash \mathrm{H}, 3.255$ $2511171,-1.9838608748,-1.029230134 \backslash \mathrm{C},-5.1560248274,-2.9349460093,4.138$ $706673 \backslash \mathrm{H},-5.1406334779,-0.993873758,5.0736007679 \backslash \mathrm{H},-5.1725876734,-3.47$ $6820093,5.0788485255 \backslash \mathrm{H},-5.1938063885,-4.7140545097,2.9267167574 \backslash \mathrm{H},-2.0$ $974601612,1.6876711575,-3.4227207442 \backslash \backslash$ Version=EM6 4L-G0 9RevA.02 $\backslash H F=-152$ $3.4732416 \backslash \mathrm{S} 2=0.75607 \backslash \mathrm{S} 2-1=0 . \backslash \mathrm{S} 2 \mathrm{~A}=0.750029 \backslash \mathrm{RMSD}=4.240 \mathrm{e}-09 \backslash \mathrm{RMSF}=2.146 \mathrm{e}-0$ $6 \backslash$ Dipole $=5.0121322,-1.0042622,-1.9265936 \backslash P G=C 01 \quad[X(C 21 H 32 N 605)] \backslash \backslash @$

\section{Table S18. M06-2X/6-31+G(d,p) Optimized Geometry of AFAR Radical $8 \mathbf{b}$.}

\# um062x/6-31+G(d,p) scf=(tight, xqc) opt geom=check pop=

none cachesize=131072 \\AAFAR z4 Arg alpha rad 3 pi-stacked rotamer $\backslash \backslash 1$, $2 \backslash \mathrm{H},-3.534975474,-3.103204683,-1.3783951149 \backslash \mathrm{N},-2.9059054068,-2.3126090$ $77,-1.42371535 \backslash \mathrm{C},-3.4265895038,-1.0022059842,-1.0347242734 \backslash \mathrm{C},-2.978298$ $8991,-0.7104817183,0.4097277328 \backslash 0,-3.6665008751,-0.9373982708,1.380450$ $0958 \backslash \mathrm{C},-4.9490374916,-0.9632980587,-1.1540728611 \backslash \mathrm{C},-5.4760755273,0.446$ $9088345,-1.0103461782 \backslash \mathrm{C},-5.4534807541,1.3124810843,-2.1087141269 \backslash \mathrm{C},-5$. $9707008787,0.9131092491,0.2100918163 \backslash C,-6.4362119463,2.2220852499,0.32$ $79331289 \backslash \mathrm{C},-5.9159914305,2.6209330817,-1.9925101308 \backslash \mathrm{H},-5.9872647579,0$. $2462254994,1.0674437193 \backslash \mathrm{N},-1.6748815023,-0.2414829658,0.4696532862 \backslash \mathrm{C},-$ $0.9096509932,-0.0414945629,1.5932307777 \backslash C, 0.5305811979,-0.0535693594,1$ $.4395603087 \backslash \mathrm{C},-1.5283203898,0.1754470644,2.9285756432 \backslash 0,1.2920800087,0$ $.2987794737,2.369558137 \backslash \mathrm{C},-1.6265427292,-2.5132437837,-1.8120858521 \backslash \mathrm{C}$, $-1.2404265251,-3.9208930042,-2.2074465389 \backslash 0,-0.8046730351,-1.586494582$ $2,-1.8317270759 \backslash \mathrm{C},-0.4350134628,-3.9341407776,-3.5068586278 \backslash \mathrm{N}, 1.061532$ $6754,-0.4136064411,0.2218272498 \backslash \mathrm{C}, 2.4873200381,-0.6986950137,0.1268020$ $019 \backslash \mathrm{C}, 2.9217350282,-1.771266995,1.1318438209 \backslash 0,4.0212576331,-1.8352129$ $277,1.616099331 \backslash 0,1.9509261876,-2.6681666718,1.3688999829 \backslash \mathrm{C}, 3.44843256$ $84,0.4959251997,0.2391037761 \backslash \mathrm{C}, 3.0273326899,1.7768347692,-0.4861482308$ $\backslash \mathrm{C}, 1.9831934941,2.6262158775,0.257701145 \backslash \mathrm{N}, 2.2592953667,2.7480342676,1$ $.6908709405 \backslash \mathrm{C}, 3.0814879035,3.6109456348,2.2524660737 \backslash \mathrm{N}, 3.2348579908,3$. $6122681238,3.587748916 \backslash \mathrm{N}, 3.7556954961,4.5165865085,1.5283294043 \backslash \mathrm{H},-2.9$ $802589484,-0.2632875917,-1.7107227779 \backslash \mathrm{H},-5.3813300066,-1.6109414855,-0$ $.3840288546 \backslash \mathrm{H},-5.2207249135,-1.3633548846,-2.1370333645 \backslash \mathrm{H},-5.086585343$ $7,0.9532397311,-3.0685083648 \backslash \mathrm{H},-1.2162083873,-0.223036888,-0.438642722$ $8 \backslash \mathrm{H},-0.754497401,0.494434407,3.6260575152 \backslash \mathrm{H},-2.3236986494,0.9241764738$ $, 2.8700742804 \backslash \mathrm{H},-1.9944248497,-0.7425828344,3.3005753331 \backslash \mathrm{H},-2.12826272$ $66,-4.5549755603,-2.2889505458 \backslash \mathrm{H},-1.0371110597,-3.5747429907,-4.345322$ $5498 \backslash \mathrm{H}, 0.4421513558,-3.2908499465,-3.4167072229 \backslash \mathrm{H},-0.1036890035,-4.949$ $0044437,-3.7345872984 \backslash \mathrm{H}, 0.4931978021,-0.9783520553,-0.4055817348 \backslash \mathrm{H}, 2.6$ 
$180766783,-1.148803333,-0.8651610949 \backslash \mathrm{H}, 2.3017578801,-3.3213206071,1.99$ $52146885 \backslash \mathrm{H}, 4.4049361857,0.1580646783,-0.168080811 \backslash \mathrm{H}, 3.6400672206,0.699$ $6789905,1.2954432309 \backslash \mathrm{H}, 3.9308970598,2.3778782952,-0.6456732767 \backslash \mathrm{H}, 2.626$ $215397,1.5548198582,-1.4817267367 \backslash \mathrm{H}, 0.9948375546,2.1716455398,0.185201$ $5092 \backslash \mathrm{H}, 1.8954525679,3.6268182515,-0.1791147305 \backslash \mathrm{H}, 2.7302397565,2.941438$ $0295,4.1492059286 \backslash \mathrm{H}, 4.0212691575,4.0747777464,4.0169180159 \backslash \mathrm{H}, 3.7127562$ $59,4.5094743459,0.5216486745 \backslash \mathrm{H}, 4.2122955938,5.2987235632,1.9713415231 \backslash$ $\mathrm{H}, 1.9319680901,1.9381895437,2.2396591681 \backslash \mathrm{C},-6.4089268237,3.0783455632$, $-0.7710088775 \backslash \mathrm{H},-5.9044539073,3.2776204595,-2.8567657293 \backslash \mathrm{H},-6.78049785$ $53,4.0940244445,-0.6798099604 \backslash \mathrm{H},-6.8287786038,2.5696687169,1.278506512$ $9 \backslash \mathrm{H},-0.632353775,-4.3178250584,-1.3857286543 \backslash \backslash$ Version=EM64L-G0 9RevA.02 $\backslash \mathrm{HF}=-1523.461656 \backslash \mathrm{S} 2=0.756516 \backslash \mathrm{S} 2-1=0 . \backslash \mathrm{S} 2 \mathrm{~A}=0.750034 \backslash \mathrm{RMSD}=4.746 \mathrm{e}-09 \backslash \mathrm{RMSF}=$ $3.664 e-06 \backslash$ Dipole $=-2.8420666,-3.7279677,-1.5922124 \backslash \mathrm{PG}=\mathrm{C} 01 \quad[\mathrm{X}(\mathrm{C} 21 \mathrm{H} 32 \mathrm{~N} 605$ ) $] \backslash \backslash @$

Table S19. M06-2X/6-31+G(d,p) Optimized Geometry of AFAR Radical 9a.

\# um062x/6-31+G(d,p) scf=(tight, xqc) opt pop=none cach

esize=131072 \\AAFAR z4 radical Arg-4 pi-stacked rotamer $\backslash \backslash 1,2 \backslash H,-2.7073$ $891994,-1.1909777938,2.5942655272 \backslash \mathrm{N},-2.1354003054,-1.0677611486,1.7703$ $220757 \backslash \mathrm{C},-2.7214333444,-0.4148340114,0.6149595177 \backslash \mathrm{C},-1.7714330074,0.64$ $02780141,0.0432234411 \backslash 0,-1.3599085643,1.5821024734,0.7286182944 \backslash \mathrm{C},-4.0$ $44382223,0.2660042298,0.9961766859 \backslash \mathrm{C},-4.637496748,0.9952123762,-0.1864$ $447975 \backslash C,-5.2264286989,0.2821265845,-1.2359968152 \backslash C,-4.5580482294,2.38$ $68864973,-0.2746950605 \backslash C,-5.0585211806,3.0564411575,-1.3906617141 \backslash C,-5$ $.7210306923,0.9475461248,-2.3553135143 \backslash \mathrm{H},-4.1074485778,2.9480693233,0$. $5406983054 \backslash \mathrm{N},-1.4535249584,0.4880480753,-1.2529139567 \backslash \mathrm{C},-0.6350499899$, $1.4459304353,-1.9848096423 \backslash \mathrm{C}, 0.6856038618,1.7092150069,-1.252924254 \backslash \mathrm{C}$, $-1.3841947983,2.7415824272,-2.2733356448 \backslash 0,1.2033851219,2.8121580923$,$1.2601917332 \backslash \mathrm{C},-0.9334454777,-1.6905590921,1.6919490244 \backslash \mathrm{C},-0.496867820$ $1,-2.5133528063,2.8837812806 \backslash 0,-0.2319969539,-1.579090382,0.6838366359$ $\backslash \mathrm{C},-0.0805990688,-3.9209400419,2.4479250792 \backslash \mathrm{N}, 1.2631488105,0.622794351$ $9,-0.6257151799 \backslash \mathrm{C}, 2.4446368956,0.7262571164,0.0790245011 \backslash \mathrm{C}, 3.500644660$ $3,1.6226859187,-0.3752640695 \backslash 0,4.2229319252,2.238577076,0.404006007 \backslash 0$, $3.6551533548,1.6761887077,-1.7002631956 \backslash C, 2.6378922418,-0.0771554755,1$ $.3255001012 \backslash \mathrm{C}, 1.8377290759,0.4202288671,2.545630952 \backslash \mathrm{C}, 1.9715469107,1.9$ $197627822,2.7987293105 \backslash N, 1.0424285077,2.629263528,1.9172500989 \backslash \mathrm{C}, 1.215$ $8665846,3.8034030246,1.33169766 \backslash \mathrm{N}, 0.1349774607,4.4525914106,0.84553064$ $97 \backslash \mathrm{N}, 2.4156388179,4.3887807907,1.2806958482 \backslash \mathrm{H},-2.9099885127,-1.1692320$ $702,-0.1610727768 \backslash \mathrm{H},-3.8445911037,0.9675959074,1.8131677053 \backslash \mathrm{H},-4.73555$ $44587,-0.5022842951,1.3623243405 \backslash \mathrm{H},-5.3162916665,-0.8006272348,-1.1696$ $888 \backslash \mathrm{H},-1.82675467,-0.3098332947,-1.7468138351 \backslash \mathrm{H},-0.749514716,3.4255613$ $026,-2.8382202868 \backslash \mathrm{H},-2.2882682349,2.5208285063,-2.8455525574 \backslash \mathrm{H},-1.6813$ $879932,3.2193868891,-1.3363028092 \backslash \mathrm{H},-1.2867596693,-2.5522724886,3.6400$ $219548 \backslash \mathrm{H},-0.9282198992,-4.4698176375,2.0294211429 \backslash \mathrm{H}, 0.700254142,-3.865$ $2204259,1.686886909 \backslash \mathrm{H}, 0.3006893187,-4.4830856101,3.302214149 \backslash \mathrm{H}, 0.68123$ $65041,-0.195214786,-0.4309222705 \backslash \mathrm{H}, 4.3488246587,2.3245886292,-1.897450$ $5297 \backslash \mathrm{H}, 3.7031556202,-0.0642658207,1.5702435101 \backslash \mathrm{H}, 2.3483332531,-1.11733$ $50104,1.1260157645 \backslash \mathrm{H}, 0.77386866,0.1817631552,2.416726788 \backslash \mathrm{H}, 2.183923774$ $4,-0.1246700165,3.4292450638 \backslash \mathrm{H}, 2.9957509084,2.2453164811,2.6042034671 \backslash$ $\mathrm{H}, 1.7212974983,2.1633194452,3.836400078 \backslash \mathrm{H},-0.732089273,3.9261968895,0$. $8606927831 \backslash \mathrm{H}, 0.2808069386,4.9676760337,-0.0133494194 \backslash \mathrm{H}, 3.2456722891,3$. $8003429542,1.2413729012 \backslash \mathrm{H}, 2.4853653157,5.3024620746,0.858445599 \backslash \mathrm{H}, 0.14$ $30119401,2.1796403956,1.745001706 \backslash C,-5.6363438659,2.3380216047,-2.4350$ $704901 \backslash \mathrm{H},-6.1870044625,0.3841780204,-3.1574457757 \backslash \mathrm{H},-6.0322634502,2.85$ $85021348,-3.3011685432 \backslash \mathrm{H},-5.0031992761,4.1395630755,-1.4412460669 \backslash \mathrm{H}, 0$. $3598709058,-1.9984069835,3.3333723394 \backslash \mathrm{H},-0.3657098667,0.9604315344,-2$. $9299544591 \backslash \backslash$ Version=EM6 4L-G09RevA. 02\HF=-1523.4916142\S2=0.756516\S2-1 $=0 . \backslash \mathrm{S} 2 \mathrm{~A}=0.750035 \backslash \mathrm{RMSD}=5.811 \mathrm{e}-09 \backslash \mathrm{RMSF}=3.990 \mathrm{e}-06 \backslash \mathrm{Dipole}=0.1449183,0.1274$ $616,0.7871489 \backslash \mathrm{PG}=\mathrm{C} 01 \quad[\mathrm{X}(\mathrm{C} 21 \mathrm{H} 32 \mathrm{~N} 605)] \backslash \backslash \mathrm{Q}$

Table S20. M06-2X/6-31+G(d,p) Optimized Geometry of AFAR Radical 9b.

\# um062x/6-31+G(d,p) scf=(xqc, tight) pop=none opt cac

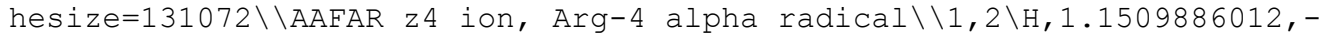


$1.9058993067,-3.9551570854 \backslash \mathrm{N}, 1.2860960599,-1.6417851821,-2.9886610644 \backslash$ $\mathrm{C}, 1.0041994884,-0.2738721086,-2.6030438138 \backslash \mathrm{C}, 0.1818055232,-0.239579952$ $9,-1.3126334387 \backslash 0,-0.8825278771,-0.8488753121,-1.2070019008 \backslash \mathrm{C}, 0.218060$ $6232,0.4433287579,-3.7105475986 \backslash C,-0.1013107876,1.8652816882,-3.313914$ $2537 \backslash \mathrm{C}, 0.8973123013,2.8451155234,-3.3290720523 \backslash \mathrm{C},-1.3807437186,2.20889$ $66888,-2.8729300285 \backslash \mathrm{C},-1.6612701748,3.5105202205,-2.4588373691 \backslash \mathrm{C}, 0.621$ $7761886,4.1444889848,-2.9094377731 \backslash \mathrm{H},-2.1609049727,1.4514822314,-2.856$ $2738252 \backslash \mathrm{N}, 0.6992776941,0.515365086,-0.3214426073 \backslash \mathrm{C},-0.015415846,0.7718$ $02661,0.9214502318 \backslash C,-0.5690540718,-0.5400478236,1.480758693 \backslash C,-1.1200$ $707994,1.8079378877,0.7525072437 \backslash 0,-1.6851054017,-0.5943431187,1.97548$ $25237 \backslash \mathrm{C}, 1.9324650803,-2.4926480855,-2.1488842065 \backslash \mathrm{C}, 2.368710876,-3.8135$ $68453,-2.755437965 \backslash 0,2.1348963756,-2.1855142811,-0.9747211174 \backslash \mathrm{C}, 3.0295$ $234795,-4.7424413454,-1.7460061161 \backslash \mathrm{N}, 0.2569160912,-1.635450312,1.40445$ $57636 \backslash C,-0.1624345252,-2.9100356557,1.7434783458 \backslash C,-1.0237587113,-3.14$ $41580773,2.8950529701 \backslash 0,-1.8420736848,-4.0536426053,2.9277882767 \backslash 0,-0$. $807502079,-2.3163866744,3.925848158 \backslash \mathrm{C}, 0.2461264112,-4.0431932896,0.861$ $8297281 \backslash \mathrm{C},-0.5073072693,-4.0943414203,-0.4821843013 \backslash \mathrm{C},-2.0123479831,-4$ $.2817817183,-0.3200254909 \backslash \mathrm{N},-2.6430836187,-3.033970742,0.1155416412 \backslash \mathrm{C}$, $-3.7310851778,-2.9824676087,0.8732136398 \backslash \mathrm{N},-4.1112808684,-1.8238901125$ $, 1.418562193 \backslash \mathrm{N},-4.4974787442,-4.0716636974,1.0524198032 \backslash \mathrm{H}, 1.952085791$, $0.2544123712,-2.4292635749 \backslash \mathrm{H},-0.705366952,-0.1174847643,-3.8902400463 \backslash$ $\mathrm{H}, 0.8176896508,0.4260646427,-4.6283149091 \backslash \mathrm{H}, 1.8935050763,2.5945122516$, $-3.6890796143 \backslash \mathrm{H}, 1.5675424552,1.0029377205,-0.4898573417 \backslash \mathrm{H},-1.597445971$ $7,2.014039116,1.7116565081 \backslash \mathrm{H},-0.6937199808,2.7302723649,0.3509465176 \backslash \mathrm{H}$ $,-1.8743885753,1.4394169935,0.0527770496 \backslash \mathrm{H}, 3.0601715299,-3.5862461572$, $-3.576001657 \backslash \mathrm{H}, 3.8647442341,-4.2442994739,-1.2500667261 \backslash \mathrm{H}, 2.323494856$, $-5.0567831672,-0.9735462861 \backslash \mathrm{H}, 3.4027526027,-5.6378169304,-2.2467270429$ $\backslash \mathrm{H}, 1.0534644251,-1.5773907807,0.7660558032 \backslash \mathrm{H},-1.4365719562,-2.54398897$ $02,4.6272245662 \backslash \mathrm{H}, 0.0917389954,-4.9805631448,1.4034488003 \backslash \mathrm{H}, 1.31718463$ $05,-3.9427841044,0.6467879512 \backslash \mathrm{H},-0.3233400538,-3.1825407407,-1.0616468$ $054 \backslash \mathrm{H},-0.120429502,-4.9383645752,-1.0639543624 \backslash \mathrm{H},-2.1930143427,-5.0380$ $539709,0.4521120204 \backslash \mathrm{H},-2.4599944925,-4.6090969221,-1.2656980518 \backslash \mathrm{H},-3.3$ $961452798,-1.1266943405,1.6207397892 \backslash \mathrm{H},-5.0005317323,-1.7621490995,1.8$ $888531525 \backslash \mathrm{H},-4.2458321909,-4.9414024124,0.61014997 \backslash \mathrm{H},-5.0481279141,-4$. $1454255835,1.8947796938 \backslash \mathrm{H},-2.2148715405,-2.1545244401,-0.1806048551 \backslash \mathrm{C}$, $-0.6603269754,4.4794807617,-2.4725564542 \backslash \mathrm{H}, 1.4023460471,4.8981238826,-$ $2.9350810158 \backslash \mathrm{H},-0.8797128717,5.4942090328,-2.1562891577 \backslash \mathrm{H},-2.663032308$ $1,3.7680596928,-2.128573908 \backslash \mathrm{H}, 1.4919672456,-4.2865153977,-3.2160845251$ $\backslash \mathrm{H}, 0.7288676184,1.1309032989,1.6419643078 \backslash \backslash$ Version=EM6 4L-G0 9RevA. 02 \HF $=-1523.4907055 \backslash \mathrm{S} 2=0.756773 \backslash \mathrm{S} 2-1=0 . \backslash \mathrm{S} 2 \mathrm{~A}=0.750037 \backslash \mathrm{RMSD}=3.010 e-09 \backslash \mathrm{RMSF}=7$. $491 e-06 \backslash$ Dipole $=-1.0080141,-0.3484092,1.6016272 \backslash \mathrm{PG}=\mathrm{C} 01 \quad[\mathrm{X}(\mathrm{C} 21 \mathrm{H} 32 \mathrm{~N} 605)] \backslash$ $\backslash$ @

Table S21. M06-2X/6-31+G(d,p) Optimized Geometry of AFAR Radical 10a.

\# um062x/6-31+G(d,p) scf=(xqc, tight) pop=none opt freq

cachesize=131072 \\AAFAR z3 ion, TS search for $\mathrm{H}$ a $-->$ b move $\backslash \backslash 1,2 \backslash \mathrm{H},-$ $1.6390847671,1.7034879539,-0.6669830134 \backslash \mathrm{N},-1.2188038379,1.0091782698,-$ $0.0631597461 \backslash C,-1.9324561146,0.6468922099,1.1601117388 \backslash C,-2.4057947492$ $,-0.8087896363,1.0417122322 \backslash 0,-2.9524272701,-1.2021402504,0.0217728213$ $\backslash C,-3.1144298217,1.5440292377,1.3694177049 \backslash \mathrm{C},-3.0028030453,2.871629458$ $7,1.8551180095 \backslash C,-1.7544896982,3.4688495617,2.1700077982 \backslash C,-4.17468953$ $02,3.6521444349,2.0351785698 \backslash C,-4.1004207604,4.949695823,2.5112014012 \backslash$ $\mathrm{C},-1.6916680105,4.76914613,2.6463021916 \backslash \mathrm{H},-5.1393679262,3.2120455261,1$ $.7978921709 \backslash \mathrm{N},-2.2071811251,-1.5846964166,2.1431099525 \backslash \mathrm{C},-2.3299768001$ $,-3.0484914325,2.0848832559 \backslash C,-1.291331391,-3.4810610622,1.0414243255 \backslash$ $C,-3.7649151688,-3.5157429315,1.8738635693 \backslash 0,-0.0979699749,-3.48273327$ $8,1.3580664184 \backslash \mathrm{C},-0.1731627198,0.2804264427,-0.5049174354 \backslash \mathrm{C}, 0.41723545$ $19,0.6974000283,-1.8365577995 \backslash 0,0.2525941636,-0.6689642387,0.170200922$ $\backslash C, 1.6258790156,-0.1359660834,-2.2385508765 \backslash N,-1.7265937034,-3.7279458$ $028,-0.209138524 \backslash \mathrm{C},-0.8781375254,-3.4252003386,-1.3616886158 \backslash \mathrm{C},-1.6670$ $485101,-2.5695231543,-2.3413813419 \backslash 0,-1.1895494096,-1.6556408181,-2.96$ $6105825 \backslash 0,-2.9321974719,-2.986604087,-2.4723349863 \backslash \mathrm{C},-0.3499227443,-4$. 
$6772999229,-2.0828892309 \backslash \mathrm{C}, 0.5827850794,-5.5586268359,-1.2434198561 \backslash \mathrm{C}$, $2.0137579631,-5.0377458703,-1.0956228143 \backslash \mathrm{N}, 2.0365862406,-3.8428560886$, $-0.2572334398 \backslash \mathrm{C}, 3.0433513716,-2.9923014166,-0.1649876682 \backslash \mathrm{N}, 2.857596093$ $,-1.8363158145,0.4804146113 \backslash \mathrm{N}, 4.2356357205,-3.2791140088,-0.7170873933$ $\backslash \mathrm{H},-1.2268768003,0.732363923,1.9978385006 \backslash \mathrm{H},-4.0910806094,1.1552570974$ $, 1.0976518563 \backslash \mathrm{H},-0.8349304702,2.9074328193,2.0297211111 \backslash \mathrm{H},-1.550043938$ $9,-1.2300889317,2.8245286716 \backslash \mathrm{H},-1.9593655994,-3.4228318926,3.041481575$ $\backslash \mathrm{H},-3.8000977784,-4.606277131,1.8014081045 \backslash \mathrm{H},-4.3675340002,-3.21033118$ $16,2.7316946179 \backslash \mathrm{H},-4.2116781713,-3.0742623504,0.9808059051 \backslash \mathrm{H},-0.373549$ $6533,0.5912765592,-2.5864466369 \backslash \mathrm{H}, 1.9613262587,0.1499314579,-3.2374994$ $542 \backslash \mathrm{H}, 2.4571488404,0.0090676597,-1.543188138 \backslash \mathrm{H}, 1.3651150562,-1.1984262$ $279,-2.2583328633 \backslash \mathrm{H},-2.717102677,-3.5797747525,-0.3691912412 \backslash \mathrm{H},-0.0613$ $757343,-2.7832962455,-1.023463805 \backslash \mathrm{H},-3.4001348365,-2.3873946471,-3.075$ $6108462 \backslash \mathrm{H}, 0.1657029083,-4.3553232085,-2.9969047502 \backslash \mathrm{H},-1.2158329634,-5$. $2718791875,-2.3931709203 \backslash \mathrm{H}, 0.1509571222,-5.7283910322,-0.2491594585 \backslash \mathrm{H}$, $0.650441937,-6.5404138478,-1.721084823 \backslash \mathrm{H}, 2.4247003177,-4.7895139985,-2$ $.0825640937 \backslash \mathrm{H}, 2.6372559077,-5.8232519643,-0.6487381155 \backslash \mathrm{H}, 1.9120738088$, $-1.4378968498,0.5195978164 \backslash \mathrm{H}, 3.6402373855,-1.2308097155,0.6711624385 \backslash \mathrm{H}$ $, 4.467469986,-4.2232332495,-0.9835519111 \backslash \mathrm{H}, 4.9649921516,-2.5855081385$, $-0.7660953261 \backslash \mathrm{H}, 1.2253973652,-3.6866995573,0.376675424 \backslash \mathrm{C},-2.8597901659$ $, 5.5167821342,2.8217137568 \backslash \mathrm{H},-0.7282266403,5.2094820766,2.8825788171 \backslash \mathrm{H}$ $,-2.8046137561,6.5334831476,3.1959257019 \backslash \mathrm{H},-5.0092773452,5.5272809867$, $2.6449800697 \backslash \mathrm{H}, 0.6739310713,1.76160466,-1.7815671097 \backslash \backslash$ Version=EM64L-G0 $9 R e v A .02 \backslash H F=-1523.4694059 \backslash S 2=0.77522 \backslash \mathrm{S} 2-1=0 . \backslash \mathrm{S} 2 \mathrm{~A}=0.750389 \backslash \mathrm{RMS} \mathrm{D}=7.269 e-$ $10 \backslash \mathrm{RMSF}=2.754 \mathrm{e}-06 \backslash \mathrm{Dipole}=-2.5818613,1.7759655,1.7332413 \backslash \mathrm{PG}=\mathrm{C} 01 \quad[\mathrm{X}(\mathrm{C} 21 \mathrm{H}$ $32 \mathrm{~N} 605)] \backslash \backslash 0$

\section{Table S22. M06-2X/6-31+G(d,p) Optimized Geometry of AFAR Radical 10b.}

$\backslash \#$ um062x/6-31+G(d,p) scf=(xqc, tight) pop=none cachesize

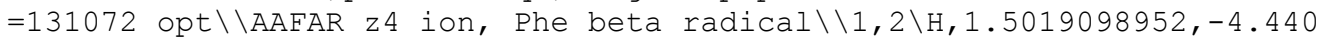
$566397,-0.0221875677 \backslash \mathrm{N}, 1.389489708,-3.869919806,-0.8477078287 \backslash \mathrm{C}, 0.1348$ $38924,-3.148150926,-1.0215720516 \backslash \mathrm{C}, 0.3610274927,-1.6307728278,-0.78864$ $84041 \backslash 0,0.0149084198,-1.0737491294,0.2513385784 \backslash \mathrm{C},-0.9207287117,-3.685$ $499859,-0.1144843344 \backslash \mathrm{C},-2.2971573174,-3.3899810347,-0.2760456428 \backslash \mathrm{C},-3$. $2428495776,-3.9286415109,0.6322917873 \backslash C,-2.7818774557,-2.5650637281,-1$ $.3228475345 \backslash C,-4.1381921138,-2.3084255996,-1.4527637312 \backslash C,-4.594972042$ $4,-3.6654489566,0.4954505875 \backslash \mathrm{H},-2.089222146,-2.1253841715,-2.036704709$ $2 \backslash \mathrm{N}, 0.9674225865,-0.9970826319,-1.8177254164 \backslash \mathrm{C}, 1.1238570684,0.44593853$ $17,-1.9554811632 \backslash \mathrm{C}, 2.140712773,1.0863781263,-1.0088433246 \backslash \mathrm{C},-0.2123154$ $925,1.1956122804,-1.8563075376 \backslash 0,2.9372000525,1.9346398071,-1.45976757$ $06 \backslash \mathrm{C}, 2.3058959992,-3.9583281233,-1.8488327938 \backslash \mathrm{C}, 3.5052768545,-4.847788$ $9878,-1.5946954117 \backslash 0,2.1707709696,-3.324433987,-2.8938088361 \backslash \mathrm{C}, 3.91957$ $75417,-5.5987629199,-2.8587621438 \backslash \mathrm{N}, 2.0737486702,0.7599620461,0.285069$ $9098 \backslash \mathrm{C}, 3.0042104165,1.2460358525,1.2834815176 \backslash \mathrm{C}, 3.6473210315,0.1116780$ $024,2.0755086401 \backslash 0,4.5246414646,0.3058230725,2.8768941185 \backslash 0,3.12747923$ $86,-1.0934472991,1.8118949295 \backslash C, 2.3424348829,2.2124453119,2.2891766701$ $\backslash \mathrm{C}, 1.7963559793,3.5176387593,1.7016702038 \backslash \mathrm{C}, 2.8574277278,4.5455229387$, $1.2993180175 \backslash \mathrm{N}, 3.5002280166,4.1334474805,0.0538572225 \backslash \mathrm{C}, 4.7161429949,4$ $.4539428458,-0.3531250682 \backslash N, 5.2556500035,3.7673054163,-1.369135561 \backslash N, 5$ $.4154529883,5.4278099351,0.2460375602 \backslash \mathrm{H},-0.160461009,-3.2751975615,-2$. $0725035906 \backslash \mathrm{H},-0.6116237909,-4.2778438689,0.7419761088 \backslash \mathrm{H},-2.8898875092$, $-4.5600764505,1.4431461659 \backslash \mathrm{H}, 1.3410267322,-1.5974336205,-2.5534545628 \backslash$ $\mathrm{H}, 1.5462455399,0.6093146197,-2.9489291305 \backslash \mathrm{H},-0.0484319231,2.2656794007$ $,-2.0118476114 \backslash \mathrm{H},-0.8867578284,0.8336628068,-2.6354361964 \backslash \mathrm{H},-0.6819282$ $826,1.036220153,-0.8837508697 \backslash \mathrm{H}, 3.3001910372,-5.5409636672,-0.77237010$ $75 \backslash \mathrm{H}, 3.135465256,-6.2907603057,-3.1760856087 \backslash \mathrm{H}, 4.0990498838,-4.8944405$ $969,-3.6726260224 \backslash \mathrm{H}, 4.8309214701,-6.1720641471,-2.6782840761 \backslash \mathrm{H}, 1.34240$ $62592,0.088804962,0.5578409588 \backslash \mathrm{H}, 3.831086844,1.7374950768,0.7636488137$ $\backslash \mathrm{H}, 3.570945957,-1.7358847221,2.389489064 \backslash \mathrm{H}, 3.0741050369,2.4305901901,3$ $.0744111504 \backslash \mathrm{H}, 1.5141132835,1.6749609088,2.7653738222 \backslash \mathrm{H}, 1.1426572361,3$. $3108493116,0.8431083264 \backslash \mathrm{H}, 1.160680776,3.9885412705,2.4567951721 \backslash \mathrm{H}, 3.61$ $57443966,4.6213343523,2.0891223367 \backslash \mathrm{H}, 2.3915350819,5.5287564589,1.16691$ 
$81198 \backslash \mathrm{H}, 4.7293203455,2.9934267714,-1.7659193995 \backslash \mathrm{H}, 6.0607434979,4.11450$ $12278,-1.8658345093 \backslash \mathrm{H}, 4.9808280998,6.0377012682,0.9206850001 \backslash \mathrm{H}, 6.38588$ $65574,5.5869754838,0.0241013923 \backslash \mathrm{H}, 3.0504641238,3.3970074498,-0.5080453$ $449 \backslash \mathrm{C},-5.0528670195,-2.8554010933,-0.5489224161 \backslash \mathrm{H},-5.3011547707,-4.092$ $3019606,1.2003863185 \backslash \mathrm{H},-6.1132484531,-2.6536190427,-0.6575103074 \backslash \mathrm{H},-4$. $4914222294,-1.6788408022,-2.263404392 \backslash \mathrm{H}, 4.3192595583,-4.1898223835,-1$. $2666317159 \backslash \backslash$ Version=EM64L-G09RevA. 02 \HF=-1523.4554258\S2=0.774959 \S2-1 $=0 . \backslash \mathrm{S} 2 \mathrm{~A}=0.75038 \backslash \mathrm{RMSD}=4.774 \mathrm{e}-09 \backslash \mathrm{RMSF}=3.033 \mathrm{e}-06 \backslash \mathrm{Dipole}=1.7811655,-5.7306$ $08,-1.2871856 \backslash \mathrm{PG}=\mathrm{C} 01 \quad[\mathrm{X}(\mathrm{C} 21 \mathrm{H} 32 \mathrm{~N} 605)] \backslash \backslash \mathrm{Q}$

Table S23. M06-2X/6-31+G(d,p) Optimized Geometry of AAWAR dication A. \# opt m062x/6-31+G(d,p) scf=(tight, xqc) cachesize $=131072$

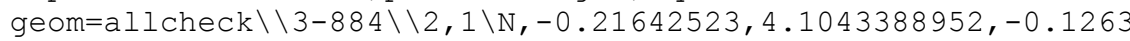
$808366 \backslash \mathrm{H}, 0.0925869514,4.9677966119,-0.5771110467 \backslash \mathrm{H},-1.2382346408,4.044$ $9995878,-0.1769090584 \backslash \mathrm{H}, 0.1999765621,3.2373422002,-0.6465254418 \backslash \mathrm{C}, 0.20$ $90315752,4.0560411736,1.302070233 \backslash \mathrm{H}, 1.3000166681,3.9658899466,1.299447$ $0895 \backslash \mathrm{C},-0.2476920472,5.3017977968,2.056940554 \backslash \mathrm{H}, 0.0850832672,5.2606544$ $88,3.0959684776 \backslash \mathrm{H},-1.3391984216,5.3782467778,2.0542647428 \backslash \mathrm{H}, 0.17467501$ $22,6.2054150841,1.6099354024 \backslash \mathrm{C},-0.4209854761,2.777193953,1.8643759082 \backslash$ $0,-1.3294834777,2.224898795,1.2462484414 \backslash \mathrm{N}, 0.0435292,2.3465242335,3.04$ $68462771 \backslash \mathrm{H}, 0.8572172134,2.7869205388,3.4533321078 \backslash \mathrm{C},-0.4645042903,1.11$ $6271828,3.6603708468 \backslash \mathrm{H}, 0.1846648709,0.9214837396,4.5190770365 \backslash \mathrm{C},-1.904$ $5943306,1.2605663292,4.1439218962 \backslash \mathrm{H},-2.588901594,1.468934429,3.3174790$ $076 \backslash \mathrm{H},-1.9672515354,2.08647694,4.8550294787 \backslash \mathrm{H},-2.2221110189,0.35416104$ $47,4.6666481072 \backslash \mathrm{C},-0.180189374,-0.0420611675,2.6890069828 \backslash 0,0.96538283$ $69,-0.2262198675,2.2751036401 \backslash \mathrm{N},-1.2030417053,-0.8363495141,2.32377266$ $93 \backslash \mathrm{H},-2.1580935454,-0.5893669092,2.5558952259 \backslash \mathrm{C},-0.9724913474,-1.92171$ $59773,1.3893966198 \backslash \mathrm{H},-0.2080900905,-2.5883040039,1.805307328 \backslash \mathrm{C},-2.2713$ $329537,-2.7298384536,1.1683166927 \backslash \mathrm{H},-2.017662846,-3.5896772404,0.53889$ $43772 \backslash \mathrm{H},-2.5759266034,-3.1290037301,2.1418542633 \backslash \mathrm{C},-3.3823023566,-1.94$ $38143525,0.5420181917 \backslash \mathrm{C},-4.4375484014,-1.3301447306,1.1732749858 \backslash \mathrm{H},-4$. $7123549429,-1.3311673083,2.2208212504 \backslash \mathrm{N},-5.2351722554,-0.7034102623,0$. $2466175183 \backslash \mathrm{H},-6.1168701671,-0.2575964673,0.4496069161 \backslash \mathrm{C},-4.7281929893$, $-0.9248755914,-1.015566179 \backslash C,-3.5595179625,-1.705338296,-0.8686553525 \backslash$ $\mathrm{C},-2.8517015801,-2.1080660715,-2.0157961343 \backslash \mathrm{H},-1.9629656595,-2.7303062$ $544,-1.9323442537 \backslash \mathrm{C},-3.3203405766,-1.7187899677,-3.2590800504 \backslash \mathrm{H},-2.800$ $2671698,-2.0384750502,-4.1564554004 \backslash C,-5.2025817248,-0.5204070704,-2.2$ $706728067 \backslash \mathrm{H},-6.1072759913,0.0704909473,-2.3721228144 \backslash \mathrm{C},-4.483966508,-0$ $.9270936003,-3.3830440901 \backslash \mathrm{H},-4.8319014931,-0.6470506781,-4.3717521802 \backslash$ $\mathrm{C},-0.4111665233,-1.4047693589,0.0633344533 \backslash 0,0.3645518993,-2.103442084$ $4,-0.6049865834 \backslash \mathrm{N},-0.8371035489,-0.204873098,-0.344525886 \backslash \mathrm{H},-1.4429496$ $156,0.3451355868,0.2570823277 \backslash \mathrm{C},-0.5292023087,0.324229004,-1.662741708$ $4 \backslash \mathrm{H},-0.4976986108,-0.5226603857,-2.3566457432 \backslash \mathrm{C},-1.6124312778,1.300085$ $7203,-2.0965616203 \backslash \mathrm{H},-1.3541387264,1.7645806931,-3.0509754986 \backslash \mathrm{H},-1.752$ $9402511,2.0768605142,-1.3396186697 \backslash \mathrm{H},-2.5560265944,0.7596824109,-2.215$ $3582509 \backslash \mathrm{C}, 0.8583073127,0.9693070861,-1.6034667424 \backslash 0,1.051250136,2.1082$ $585948,-1.1236803888 \backslash \mathrm{C}, 3.8022444058,0.2029497672,-3.4533308949 \backslash 0,3.298$ $3308356,-0.6585081776,-4.1283471666 \backslash 0,4.9270282816,0.851432504,-3.7487$ $209835 \backslash \mathrm{H}, 5.2738259505,0.5221292906,-4.5952366432 \backslash \mathrm{N}, 1.8565764563,0.2218$ $932865,-2.0576546337 \backslash \mathrm{H}, 1.642830392,-0.6993995857,-2.4306453778 \backslash \mathrm{C}, 3.249$ $6082591,0.6300191634,-2.0996853321 \backslash \mathrm{H}, 3.2987850939,1.716860189,-1.99569$ $18526 \backslash \mathrm{C}, 4.0582293086,-0.068557397,-0.9919250623 \backslash \mathrm{H}, 3.9199609104,-1.1499$ $083571,-1.1316201434 \backslash \mathrm{H}, 5.1214539094,0.1433524173,-1.1449866172 \backslash \mathrm{C}, 3.624$ $8428336,0.3428194724,0.4168332216 \backslash \mathrm{H}, 2.546325828,0.5408667858,0.4540373$ $098 \backslash \mathrm{H}, 4.1307092533,1.2679991752,0.7129012983 \backslash \mathrm{C}, 3.928240942,-0.74492473$ $35,1.4496077935 \backslash \mathrm{H}, 4.9702991245,-1.0657964546,1.3738712503 \backslash \mathrm{H}, 3.75471777$ $51,-0.3778160092,2.4633776822 \backslash \mathrm{N}, 3.0427586095,-1.8895306102,1.227360739$ $8 \backslash \mathrm{H}, 2.0518737123,-1.6599822244,1.2930178139 \backslash \mathrm{C}, 3.3685412868,-3.09073354$ $28,0.7616028571 \backslash \mathrm{N}, 4.6335882497,-3.535952057,0.8305134558 \backslash \mathrm{H}, 5.281507026$ $8,-3.1539229639,1.5022821883 \backslash \mathrm{H}, 4.8962253588,-4.3989265179,0.3782615395$ $\backslash \mathrm{N}, 2.4226790504,-3.8557771111,0.2148302603 \backslash \mathrm{H}, 2.5913750478,-4.833013781$ $, 0.0298409056 \backslash \mathrm{H}, 1.5544463562,-3.4209899869,-0.1113659213 \backslash \backslash$ Version=EM64 
$\mathrm{L}-\mathrm{G} 0$ 9RevA. 02 $\backslash$ State $=1-\mathrm{A} \backslash \mathrm{HF}=-1958.576747 \backslash \mathrm{RMSD}=4.353 \mathrm{e}-09 \backslash \mathrm{RMSF}=5.191 \mathrm{e}-06 \backslash \mathrm{D}$ ipole=2.699319,2.2979172,2.9684552\Quadrupole=17.7230267,13.2403857,-3 $0.9634123,-27.210186,-4.5881155,3.0816205 \backslash \mathrm{PG}=\mathrm{C} 01 \quad[\mathrm{X}(\mathrm{C} 26 \mathrm{H} 41 \mathrm{~N} 906)] \backslash \backslash @$

Table S24. M06-2X/6-31+G(d,p) Optimized Geometry of AAWAR dication B. \# opt m062x/6-31+G(d,p) scf=(tight, xqc) cachesize=13107 2 geom=allcheck $\backslash \backslash 4-353$ B3LYP opt $\backslash \backslash 2,1 \backslash N,-1.3666752724,4.2727490954,0.2$ $083425304 \backslash \mathrm{H},-1.7975836641,3.3358684629,0.5574032245 \backslash \mathrm{H},-2.0602110463,5$. $0220966171,0.2487984573 \backslash \mathrm{H},-0.5786913171,4.5085360215,0.8193310042 \backslash \mathrm{C},-0$ $.8656997092,4.0674365707,-1.1820563149 \backslash \mathrm{H},-1.7157093702,3.7023510808,-1$ $.7670495186 \backslash \mathrm{C},-0.2997677617,5.3604339442,-1.7623849145 \backslash \mathrm{H}, 0.5532181271$, $5.7105815346,-1.1734383115 \backslash \mathrm{H},-1.0625246086,6.1429712103,-1.7884034158 \backslash$ $\mathrm{H}, 0.0424682038,5.1990846079,-2.7866170371 \backslash \mathrm{C}, 0.1955000978,2.9683985958$, $-1.0591985437 \backslash 0,0.6464413071,2.6852077144,0.0501282539 \backslash \mathrm{N}, 0.5959893596$, $2.3879498019,-2.1986688903 \backslash \mathrm{H}, 0.1194417405,2.6052782983,-3.0627551832 \backslash \mathrm{C}$ $, 1.574749312,1.295819278,-2.1923305121 \backslash \mathrm{H}, 1.5576729594,0.8738443664,-3$. $2018844632 \backslash \mathrm{C}, 2.982391694,1.7887305292,-1.8689684399 \backslash \mathrm{H}, 3.2793636627,2.5$ $415900893,-2.6018301533 \backslash \mathrm{H}, 3.709234581,0.972175067,-1.9279844868 \backslash \mathrm{H}, 3.03$ $15384757,2.233747913,-0.8721769808 \backslash \mathrm{C}, 1.0165052932,0.1844093277,-1.2869$ $068586 \backslash 0,-0.1356495413,-0.2266311615,-1.4521699817 \backslash \mathrm{N}, 1.8120520347,-0.2$ $98633799,-0.3208465485 \backslash \mathrm{H}, 2.7816426462,-0.0048219338,-0.249283764 \backslash \mathrm{C}, 1.3$ $471191574,-1.3785557622,0.5307644229 \backslash \mathrm{H}, 1.1357523381,-2.2629832075,-0.0$ $842064924 \backslash \mathrm{C}, 2.4148767417,-1.7361187889,1.5924918904 \backslash \mathrm{H}, 2.4353204371,-0$. $9454297722,2.3526495105 \backslash \mathrm{H}, 2.0872027924,-2.6516774239,2.0932500581 \backslash \mathrm{C}, 3$. $7801433,-1.8945010452,0.9991278119 \backslash \mathrm{C}, 4.364500464,-3.0335430774,0.50518$ $76795 \backslash \mathrm{H}, 3.9952181842,-4.0500491132,0.4948076219 \backslash \mathrm{N}, 5.6010984523,-2.7353$ $815714,-0.009647542 \backslash \mathrm{H}, 6.2484831038,-3.409185162,-0.3895866119 \backslash \mathrm{C}, 5.8522$ $087033,-1.3913894675,0.1445928208 \backslash \mathrm{C}, 4.7253760136,-0.8261918047,0.78690$ $4774 \backslash \mathrm{C}, 4.7294372444,0.5495881472,1.0913050356 \backslash \mathrm{H}, 3.8967602442,1.0073477$ $952,1.624187147 \backslash \mathrm{C}, 5.8306951452,1.3099596612,0.7338603906 \backslash \mathrm{H}, 5.857507068$ $2,2.3676451141,0.9753136108 \backslash C, 6.967043219,-0.6266483861,-0.2178071882 \backslash$ $\mathrm{H}, 7.8270689548,-1.0748601779,-0.7049138244 \backslash \mathrm{C}, 6.9361793228,0.7256801823$ $, 0.0801603347 \backslash \mathrm{H}, 7.7872305075,1.3456218454,-0.1807393768 \backslash \mathrm{C}, 0.035919278$, $-0.9984180185,1.217773168 \backslash 0,-0.8382655922,-1.8456255707,1.4353999052 \backslash \mathrm{N}$ $,-0.0762802653,0.2748514681,1.6330295657 \backslash \mathrm{H}, 0.5758776775,0.9692836387,1$ $.2769255981 \backslash \mathrm{C},-1.252631258,0.7458170693,2.3479859912 \backslash \mathrm{H},-1.5482370536,-$ $0.0457042305,3.0430110298 \backslash \mathrm{C},-0.9192095683,2.0172879181,3.1161242788 \backslash \mathrm{H}$, $-1.8126731822,2.431155245,3.5887949805 \backslash \mathrm{H},-0.4868789146,2.7629924424,2$. $4428911053 \backslash \mathrm{H},-0.1860698662,1.7959608532,3.893993818 \backslash \mathrm{C},-2.4047659115,0$. $9739124354,1.3602740687 \backslash 0,-2.4851543492,1.9986749846,0.649617717 \backslash \mathrm{C},-5$. $6625028512,-0.4447663671,1.3853088373 \backslash 0,-5.4865688883,-1.1343640727,2$. $3576444732 \backslash 0,-6.8496343475,-0.0772248075,0.9080511275 \backslash \mathrm{H},-7.5509693954$, $-0.4505523848,1.468527553 \backslash \mathrm{N},-3.3132926307,0.0067539679,1.3014205108 \backslash \mathrm{H}$, $-3.2001450317,-0.8059777242,1.9016843326 \backslash \mathrm{C},-4.525947848,0.0528339371,0$ $.5016174148 \backslash \mathrm{H},-4.7052038618,1.0876218074,0.1989608532 \backslash \mathrm{C},-4.4163606866$, $-0.8698889153,-0.7250968339 \backslash \mathrm{H},-4.1986940368,-1.8778037056,-0.345076148$ $\backslash \mathrm{H},-5.3926786373,-0.9163548352,-1.2180669506 \backslash \mathrm{C},-3.3303463666,-0.427764$ $8475,-1.7087027194 \backslash \mathrm{H},-2.5129368423,0.0850970343,-1.1883270016 \backslash \mathrm{H},-3.741$ $5316529,0.2798816013,-2.4361754087 \backslash \mathrm{C},-2.7219556913,-1.6135192994,-2.45$ $98540702 \backslash \mathrm{H},-3.5081155349,-2.2185405684,-2.9186839287 \backslash \mathrm{H},-2.0488360194,-$ $1.2694405377,-3.2480870238 \backslash \mathrm{N},-1.9495151688,-2.4361516736,-1.5271166082$ $\backslash \mathrm{H},-1.2182423996,-1.9245697494,-1.0336341013 \backslash \mathrm{C},-2.2111597284,-3.675995$ $4002,-1.1286907676 \backslash \mathrm{N},-3.0389437898,-4.4675967528,-1.8299335411 \backslash \mathrm{H},-3.23$ $73056429,-4.2840936575,-2.8011255671 \backslash \mathrm{H},-3.3132842509,-5.3682550244,-1$. $4673663037 \backslash \mathrm{N},-1.6453608679,-4.1339047578,-0.0105662176 \backslash \mathrm{H},-1.2428446903$ $,-3.4681118788,0.65473767 \backslash \mathrm{H},-1.689762731,-5.1125408405,0.2287179682 \backslash \backslash \mathrm{V}$ ersion=EM6 4L-G0 9RevA. 02 State $=1-A \backslash H F=-1958.5732517 \backslash$ RMSD $=2.078 e-09 \backslash$ RMSF $=1.093 e-05 \backslash \mathrm{Dipole}=-2.7241151,0.6518583,-2.8952407 \backslash$ Quadrupole=14.447643 $7,36.7764683,-51.224112,0.0293268,1.0782638,6.4065865 \backslash P G=C 01 \quad[X(C 26 H 41$ N906) ] \\@

Table S25. M06-2X/6-31+G(d,p) Optimized Geometry of AAWAR dication C. 
\# opt m062x/6-31+G(d,p) scf=(tight, xqc) cachesize=131072 g

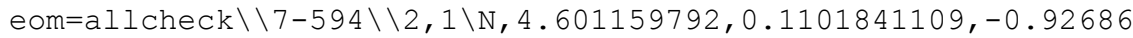
$3816 \backslash \mathrm{H}, 5.3557011489,0.7959560862,-0.8475959772 \backslash \mathrm{H}, 3.7491814622,0.532633$ $7668,-0.5011932729 \backslash \mathrm{H}, 4.3831757348,-0.0891415489,-1.9112554151 \backslash \mathrm{C}, 4.8978$ $824976,-1.1841362604,-0.2382403465 \backslash \mathrm{H}, 5.848569252,-1.550135227,-0.64146$ $69852 \backslash \mathrm{C}, 4.9919841157,-0.9726861949,1.2656058382 \backslash \mathrm{H}, 5.7168718451,-0.1870$ $675648,1.4952273762 \backslash \mathrm{H}, 5.3519540515,-1.880094178,1.7560031291 \backslash \mathrm{H}, 4.02015$ $59961,-0.6917939277,1.6793762184 \backslash \mathrm{C}, 3.7932159497,-2.1325721418,-0.73368$ $23506 \backslash 0,3.2594656743,-1.9032229149,-1.8167700911 \backslash N, 3.5011456241,-3.195$ $656056,0.0286486387 \backslash \mathrm{H}, 3.897805972,-3.2949584961,0.9537045168 \backslash \mathrm{C}, 2.46728$ $84557,-4.1514109807,-0.3797074873 \backslash \mathrm{H}, 2.5882937751,-4.3152375211,-1.4545$ $124794 \backslash \mathrm{C}, 2.616849088,-5.4521730658,0.3918564661 \backslash \mathrm{H}, 1.8622802539,-6.1689$ $698284,0.0622056412 \backslash \mathrm{H}, 2.4685274797,-5.2800597311,1.4609175587 \backslash \mathrm{H}, 3.6038$ $759958,-5.884649125,0.2147317502 \backslash \mathrm{C}, 1.1091465984,-3.4938886912,-0.08772$ $99295 \backslash 0,0.6621897817,-3.47980572,1.0578976318 \backslash \mathrm{N}, 0.5027160501,-2.913681$ $4479,-1.1466067105 \backslash \mathrm{H}, 1.0673965541,-2.80549019,-1.9817508449 \backslash \mathrm{C},-0.77261$ $93584,-2.1951555114,-1.1073088093 \backslash \mathrm{H},-1.0164639935,-1.9474380491,-2.142$ $4933201 \backslash \mathrm{C},-1.9299876015,-3.0362624329,-0.5265528757 \backslash \mathrm{H},-1.9626586026,-3$ $.9715518888,-1.0941205386 \backslash \mathrm{H},-1.7159631349,-3.3014496618,0.512283741 \backslash \mathrm{C}$, $-3.2453548887,-2.3204882553,-0.6279645234 \backslash C,-4.1203572078,-2.377139728$ $1,-1.6832662048 \backslash \mathrm{H},-4.0491761239,-2.9398209964,-2.6046076495 \backslash \mathrm{N},-5.22117$ $77282,-1.5963039636,-1.4291614801 \backslash \mathrm{H},-6.0332281423,-1.5307706049,-2.024$ $2929855 \backslash C,-5.0956926831,-1.0262648906,-0.1864907445 \backslash C,-3.8550567361,-1$ $.4567656259,0.3528969338 \backslash \mathrm{C},-3.4971676549,-1.0341988229,1.6482524048 \backslash \mathrm{H}$, $-2.5738256238,-1.3809640291,2.1034797811 \backslash \mathrm{C},-4.3536128985,-0.1985915927$ $, 2.347524442 \backslash \mathrm{H},-4.1005329121,0.1119986807,3.3560578319 \backslash \mathrm{C},-5.9668467749$ $,-0.1810722688,0.5147904717 \backslash \mathrm{H},-6.9246953887,0.114881803,0.0973589871 \backslash \mathrm{C}$ $,-5.5768888027,0.2293401376,1.7834740342 \backslash \mathrm{H},-6.2421916194,0.856637727,2$ $.3683435099 \backslash \mathrm{C},-0.6309661584,-0.8441231108,-0.4036668615 \backslash 0,-0.878080653$ $5,0.2211461502,-0.9946803824 \backslash \mathrm{N},-0.2299770786,-0.8595512185,0.874416439$ $5 \backslash \mathrm{H}, 0.0454777846,-1.7551672478,1.2861888409 \backslash \mathrm{C}, 0.0931970609,0.382315620$ $5,1.543421645 \backslash \mathrm{H},-0.8004441324,1.022461159,1.5573454449 \backslash \mathrm{C}, 0.5680171701$, $0.1035976678,2.966543189 \backslash \mathrm{H}, 0.8276427155,1.0337914736,3.4768632867 \backslash \mathrm{H}, 1$. $4472580862,-0.5466726733,2.9491576981 \backslash \mathrm{H},-0.2239611682,-0.391027616,3.5$ $33806371 \backslash \mathrm{C}, 1.1984846692,1.0712576181,0.7378482883 \backslash 0,2.1871060913,0.438$ $2423369,0.3570830722 \backslash \mathrm{C}, 3.311425675,3.2845524382,0.3675633751 \backslash 0,4.34364$ $54603,2.7382239226,0.0460595913 \backslash 0,3.1944416366,4.1135000684,1.39829520$ $48 \backslash \mathrm{H}, 4.0574868427,4.2261617774,1.8316432244 \backslash \mathrm{N}, 1.0465478975,2.382381075$ $5,0.4789402903 \backslash \mathrm{H}, 0.2113157657,2.8586367498,0.7948050155 \backslash \mathrm{C}, 1.9881530319$ $, 3.0907073221,-0.3722065554 \backslash \mathrm{H}, 2.2213779176,2.4463166321,-1.2275062724 \backslash$ $\mathrm{C}, 1.4186200416,4.4226309122,-0.8685598373 \backslash \mathrm{H}, 1.0740407177,5.0144005569$, $-0.0110107714 \backslash \mathrm{H}, 2.2562213971,4.9823480769,-1.296465575 \backslash \mathrm{C}, 0.3217370631$, $4.3053656851,-1.9464162557 \backslash \mathrm{H}, 0.4758658531,3.4080668011,-2.5597744197 \backslash \mathrm{H}$ $, 0.4363073525,5.1519569283,-2.6285055767 \backslash C,-1.130946807,4.3548738693,-$ $1.4719003924 \backslash \mathrm{H},-1.2303456499,5.1542632368,-0.7268783231 \backslash \mathrm{H},-1.775237602$ $9,4.6008992326,-2.3245217252 \backslash \mathrm{N},-1.5710380973,3.0859520066,-0.881749373$ $7 \backslash \mathrm{H},-1.0726474195,2.2319039884,-1.136953522 \backslash \mathrm{C},-2.8290136809,2.88436827$ $44,-0.4497199071 \backslash \mathrm{N},-3.6312813057,3.9275206189,-0.2068533386 \backslash \mathrm{H},-3.32907$ $68016,4.8780450668,-0.3485768331 \backslash \mathrm{H},-4.5880685089,3.7812278461,0.079623$ $6382 \backslash \mathrm{N},-3.2570048495,1.6497005718,-0.2432500604 \backslash \mathrm{H},-4.1454611304,1.4464$ $792336,0.2062606718 \backslash \mathrm{H},-2.6589044579,0.8651937703,-0.4946813689 \backslash \backslash$ Versio $\mathrm{n}=\mathrm{EM} 64 \mathrm{~L}-\mathrm{G} 0$ 9RevA $.02 \backslash \mathrm{State}=1-\mathrm{A} \backslash \mathrm{HF}=-1958.5703507 \backslash \mathrm{RMSD}=4.585 \mathrm{e}-09 \backslash \mathrm{RMSF}=6.89$ $6 e-06 \backslash$ Dipole $=1.4540859,3.5877505,-0.9616347 \backslash$ Quadrupole $=49.6178361,7.60$ $27268,-57.2205629,-23.0629137,11.2710372,2.6784432 \backslash P G=C 01 \quad[X(C 26 H 41 N 90$ $6)] \backslash \backslash 0$

Table S26. M06-2X/6-31+G(d,p) Optimized Geometry of AAWAR dication D. \# opt m062x/6-31+G(d,p) scf=(tight, xqc) cachesize=13107

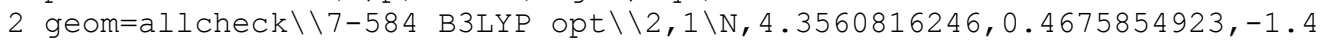
$02410979 \backslash \mathrm{H}, 5.0246812961,1.2392646271,-1.4627268691 \backslash \mathrm{H}, 3.5346946107,0.81$ $44002229,-0.8642127416 \backslash \mathrm{H}, 4.0234317302,0.1963090209,-2.3369549271 \backslash \mathrm{C}, 4.9$ $120304271,-0.7499469064,-0.7328938024 \backslash \mathrm{H}, 5.8344017344,-1.0096334684,-1$. 
$2641195877 \backslash \mathrm{C}, 5.1968345902,-0.4693865516,0.7342864351 \backslash \mathrm{H}, 5.7356208391,-1$ $.3073059817,1.1830680904 \backslash \mathrm{H}, 4.269874737,-0.2871311947,1.2833347485 \backslash \mathrm{H}, 5$. $8455611421,0.4050317915,0.8342363869 \backslash \mathrm{C}, 3.8886239049,-1.8518456678,-1.0$ $539020792 \backslash 0,3.254136641,-1.7673078601,-2.1040703242 \backslash \mathrm{N}, 3.7766616585,-2$. $8736238107,-0.194268697 \backslash \mathrm{H}, 4.2625808757,-2.8560899933,0.6928050716 \backslash \mathrm{C}, 2$. $8579911334,-3.9919016777,-0.447486607 \backslash \mathrm{H}, 2.9075119822,-4.2074503747,-1$. $5190691279 \backslash \mathrm{C}, 3.263943519,-5.202412343,0.3744205509 \backslash \mathrm{H}, 3.1895777653,-4.9$ $823444384,1.4423531159 \backslash \mathrm{H}, 4.2826484622,-5.5054870104,0.1235516321 \backslash \mathrm{H}, 2.5$ $929490909,-6.0368930737,0.1625013514 \backslash \mathrm{C}, 1.448095336,-3.5062484713,-0.06$ $87942098 \backslash 0,1.0450784971,-3.5879371649,1.0893271272 \backslash \mathrm{N}, 0.7515259496,-2.9$ $388730692,-1.0777767945 \backslash \mathrm{H}, 1.2703586348,-2.762697077,-1.9311218693 \backslash \mathrm{C},-0$ $.5419732976,-2.2664310129,-0.9512461768 \backslash \mathrm{H},-0.833680153,-1.9817601923,-$ $1.9641744044 \backslash \mathrm{C},-1.6646828069,-3.1488083354,-0.3652698869 \backslash \mathrm{H},-1.66530202$ $92,-4.0899285823,-0.9240238833 \backslash \mathrm{H},-1.4503166482,-3.3979639866,0.6779076$ $04 \backslash C,-2.996670182,-2.4668142713,-0.4964109354 \backslash C,-3.849693554,-2.574332$ $7798,-1.5642753545 \backslash \mathrm{H},-3.7583038961,-3.1815924264,-2.4550408804 \backslash \mathrm{N},-4.95$ $27739018,-1.7761729821,-1.3759054647 \backslash \mathrm{H},-5.7579891659,-1.7525916693,-1$. $9832376831 \backslash \mathrm{C},-4.8505342323,-1.1450237475,-0.1627347135 \backslash \mathrm{C},-3.6225742641$ $,-1.5518312252,0.4253494555 \backslash \mathrm{C},-3.2990391318,-1.0729970654,1.7090334066$ $\backslash \mathrm{H},-2.388499419,-1.4002712203,2.203608869 \backslash \mathrm{C},-4.1760996878,-0.210442262$ $6,2.3537146986 \backslash \mathrm{H},-3.9512091486,0.1396914692,3.3559205758 \backslash \mathrm{C},-5.74172042$ $09,-0.2766914775,0.4820194548 \backslash \mathrm{H},-6.7012386555,-0.0236769968,0.03804769$ $72 \backslash \mathrm{C},-5.3879460003,0.1875905943,1.7434753119 \backslash \mathrm{H},-6.0735481588,0.8268207$ $283,2.2913717946 \backslash C,-0.4078095821,-0.943097084,-0.1965870943 \backslash 0,-0.67954$ $77071,0.1367702164,-0.7400802025 \backslash \mathrm{N}, 0.0271860271,-1.0063160167,1.072522$ $0101 \backslash \mathrm{H}, 0.3280752173,-1.9137403088,1.4358035163 \backslash \mathrm{C}, 0.3452374945,0.204068$ $9289,1.8002083375 \backslash \mathrm{H},-0.5836120385,0.7483711439,2.0256485439 \backslash \mathrm{C}, 1.079005$ $2152,-0.1448235786,3.0925164201 \backslash \mathrm{H}, 1.3262626864,0.7584869492,3.65452125$ $13 \backslash \mathrm{H}, 2.0023909194,-0.6841048522,2.8620021892 \backslash \mathrm{H}, 0.4507753172,-0.7797195$ $138,3.7219543483 \backslash \mathrm{C}, 1.2255993231,1.0772611449,0.9017555538 \backslash 0,2.17102543$ $77,0.5913994027,0.2766718769 \backslash \mathrm{C}, 2.9982034012,3.4922845109,0.1649265217 \backslash$ $0,3.9243676431,3.0694666791,-0.4922247386 \backslash 0,3.1394708568,4.2201590723$, $1.2661996284 \backslash \mathrm{H}, 4.0827867621,4.3753439443,1.4413502281 \backslash \mathrm{N}, 0.9212921755,2$ $.3866900672,0.8356992649 \backslash \mathrm{H}, 0.1397807669,2.7389150006,1.3713817493 \backslash \mathrm{C}, 1$. $5290697396,3.2358081369,-0.1761539649 \backslash \mathrm{H}, 1.5416239072,2.6714627103,-1.1$ $162614386 \backslash \mathrm{C}, 0.7537644626,4.5515079798,-0.3358076057 \backslash \mathrm{H}, 0.9526852495,5.1$ $920694918,0.5290884423 \backslash \mathrm{H}, 1.1626433779,5.068015512,-1.2110194438 \backslash \mathrm{C},-0.7$ $63782229,4.3854655891,-0.4883141238 \backslash \mathrm{H},-1.189255284,5.3738174998,-0.690$ $0009302 \backslash \mathrm{H},-1.2163844208,4.0556346048,0.4552378722 \backslash \mathrm{C},-1.1941493365,3.42$ $15551869,-1.5970838183 \backslash \mathrm{H},-0.8498274262,3.7697767393,-2.5743154611 \backslash \mathrm{H},-0$ $.7802976703,2.4177960241,-1.4552671648 \backslash N,-2.6561712847,3.3233526885,-1$ $.675095134 \backslash \mathrm{H},-3.1364183575,3.9236318923,-2.3311375002 \backslash \mathrm{C},-3.4053315553$, $2.6298210417,-0.8094360137 \backslash \mathrm{N},-4.7242354915,2.8417569596,-0.7445980158 \backslash$ $\mathrm{H},-5.1611052688,3.6407882485,-1.1784321153 \backslash \mathrm{H},-5.3036711688,2.194683597$ $7,-0.2233644113 \backslash \mathrm{N},-2.8439864077,1.7421742866,0.0069877734 \backslash \mathrm{H},-3.4403506$ $72,1.246349362,0.6648503971 \backslash \mathrm{H},-1.9871715077,1.2489891638,-0.2675621266$ $\backslash \backslash$ Version=EM6 4L-G0 9RevA. 02 \State=1-A \HF=-1958.5711179 $\backslash$ RMSD=8.647e-09\R $\mathrm{MSF}=1.078 \mathrm{e}-05 \backslash \mathrm{Dipole}=0.6188924,3.5133128,-1.527916 \backslash$ Quadrupole $=59.14210$ $45,-4.7368904,-54.4052141,-23.3811331,13.8832607,-0.6743265 \backslash \mathrm{PG}=\mathrm{C} 01$ [X( C26H41N906) ] \\@

Table S27. M06-2X/6-31+G(d,p) Optimized Geometry of AAWAR dication E. opt $\mathrm{m062x} / 6-31+\mathrm{G}(\mathrm{d}, \mathrm{p}) \quad \mathrm{scf}=(\mathrm{tight}, \mathrm{xqc}) \quad$ cachesize $=13107$ 2 geom=allcheck $\backslash \backslash 2-746 \backslash \backslash 2,1 \backslash N, 6.5731791337,-0.1931928626,0.9$ $28110622 \backslash \mathrm{H}, 6.4137219192,-1.0965145019,0.4481636964 \backslash \mathrm{H}, 5.8674527932,-0.1$ $291407228,1.6909469805 \backslash \mathrm{H}, 7.512611739,-0.2160972758,1.3293192335 \backslash \mathrm{C}, 6.34$ $87144795,0.985838638,0.0273698275 \backslash \mathrm{H}, 7.1437215914,1.7087538167,0.233492$ $4955 \backslash \mathrm{C}, 6.3550982968,0.5614000998,-1.4363801081 \backslash \mathrm{H}, 7.2637157492,-0.00082$ $98041,-1.6693691884 \backslash \mathrm{H}, 6.3493045659,1.4373084998,-2.0888114353 \backslash \mathrm{H}, 5.4789$ $334177,-0.0541022872,-1.6642641282 \backslash \mathrm{C}, 5.0123630212,1.5881567738,0.50095$ $60947 \backslash 0,4.5004242557,1.1579158686,1.5358110098 \backslash \mathrm{N}, 4.4924541647,2.569302$ $4518,-0.2410880944 \backslash \mathrm{H}, 4.9423806519,2.8512001456,-1.1027002969 \backslash \mathrm{C}, 3.25015$ 
$06281,3.2654771387,0.1277216226 \backslash \mathrm{H}, 3.225507316,3.2963135442,1.222401128$ $1 \backslash \mathrm{C}, 3.2438419167,4.6651635038,-0.4595998043 \backslash \mathrm{H}, 4.103310601,5.2327645576$ $,-0.0968736558 \backslash \mathrm{H}, 2.3312212979,5.1844991621,-0.1640437004 \backslash \mathrm{H}, 3.253372070$ $9,4.6266989031,-1.552064267 \backslash C, 2.0525064316,2.4360424295,-0.3731655017 \backslash$ $0,1.3486096416,2.8111192694,-1.3037651035 \backslash \mathrm{N}, 1.8657641059,1.2629781922$, $0.2692326673 \backslash \mathrm{H}, 2.4751967952,1.0253508897,1.0458881802 \backslash \mathrm{C}, 0.9237655318,0$ $.2602656787,-0.2103168631 \backslash \mathrm{H}, 0.8838146094,0.353276577,-1.3029072109 \backslash \mathrm{C}, 1$ $.3898653321,-1.1352844074,0.1968117586 \backslash \mathrm{H}, 1.4785872382,-1.1686361357,1$. $2882684782 \backslash \mathrm{H}, 0.592779892,-1.8432276447,-0.0506459711 \backslash \mathrm{C}, 2.6814083513,-1$ $.5584970227,-0.4491921967 \backslash \mathrm{C}, 2.946567914,-1.6409733415,-1.7918534918 \backslash \mathrm{H}$, $2.3271997869,-1.3701762448,-2.63671905 \backslash N, 4.1919935231,-2.1996934464,-2$ $.001011874 \backslash \mathrm{H}, 4.5558691117,-2.4696964119,-2.902943378 \backslash \mathrm{C}, 4.7485065053,-2$ $.5245184729,-0.788248342 \backslash \mathrm{C}, 3.833091721,-2.1142849948,0.2176977629 \backslash \mathrm{C}, 4$. $1518762761,-2.358214358,1.5672469467 \backslash \mathrm{H}, 3.4642188222,-2.0731942642,2.35$ $83368393 \backslash \mathrm{C}, 5.3451114334,-2.9964138532,1.8751374041 \backslash \mathrm{H}, 5.5848150213,-3.2$ $200163024,2.9096390312 \backslash \mathrm{C}, 5.9657910841,-3.1543808987,-0.4837428071 \backslash \mathrm{H}, 6$. $643964498,-3.4848844257,-1.2650836717 \backslash C, 6.2438452629,-3.3938277147,0.8$ $581900095 \backslash \mathrm{H}, 7.1569941333,-3.9152722617,1.1273005771 \backslash \mathrm{C},-0.4799264124,0$. $5501978135,0.3441294216 \backslash 0,-0.998163848,-0.1246084168,1.2179359628 \backslash \mathrm{N},-1$ $.1172831526,1.6113719648,-0.2307879285 \backslash \mathrm{H},-0.5860072618,2.2137173277,-0$ $.8567310875 \backslash \mathrm{C},-2.3756224494,2.0731865566,0.3272402167 \backslash \mathrm{H},-2.2745073366$, $2.2103370518,1.4101337614 \backslash \mathrm{C},-2.8056868591,3.3800496118,-0.3378692687 \backslash \mathrm{H}$ $,-3.7572123205,3.7145608159,0.0788962101 \backslash \mathrm{H},-2.9211834047,3.2467399992$, $-1.4176895162 \backslash \mathrm{H},-2.058444519,4.1565815808,-0.1575949199 \backslash \mathrm{C},-3.475534602$ $9,1.027455127,0.1731564118 \backslash 0,-4.4614520273,1.0389015707,0.9266759972 \backslash \mathrm{C}$ $,-3.4444711753,-2.0942811582,-1.630950407 \backslash 0,-2.3068891767,-1.972922858$ $7,-2.0151061205 \backslash 0,-4.1785451655,-3.1965677909,-1.7861642599 \backslash \mathrm{H},-3.65009$ $36365,-3.8685868792,-2.2474879758 \backslash N,-3.3553774818,0.1485520639,-0.8281$ $499755 \backslash \mathrm{H},-2.4816638588,0.1102451366,-1.3428436701 \backslash \mathrm{C},-4.2287111696,-0.9$ $998908079,-0.9223940458 \backslash \mathrm{H},-4.4284069979,-1.3619080232,0.096944285 \backslash \mathrm{C},-5$ $.5719899479,-0.705131038,-1.6373166277 \backslash \mathrm{H},-5.7991423446,0.3567181759,-1$ $.4843186294 \backslash \mathrm{H},-5.435451535,-0.8285888898,-2.7154133474 \backslash \mathrm{C},-6.7340093219$ $,-1.5874929895,-1.1356765538 \backslash \mathrm{H},-6.3408797823,-2.4762910281,-0.63205059$ $97 \backslash \mathrm{H},-7.323644739,-1.9521859965,-1.9799678374 \backslash \mathrm{C},-7.6940582598,-0.85454$ $06027,-0.1968138925 \backslash \mathrm{H},-8.2682470176,-0.1008305063,-0.7517394922 \backslash \mathrm{H},-8.3$ $933577746,-1.5840889686,0.2256663648 \backslash N,-6.9530244315,-0.2052364346,0.8$ $842317622 \backslash \mathrm{H},-5.9830876531,0.0854811925,0.7138985101 \backslash \mathrm{C},-7.4968699258,0$. $3572285226,1.9570095236 \backslash \mathrm{N},-8.7927636523,0.1862539529,2.2461148348 \backslash \mathrm{H},-9$ $.387123614,-0.3845479251,1.6665343989 \backslash \mathrm{H},-9.2104906898,0.61768258,3.056$ $1013761 \backslash \mathrm{N},-6.7247202493,1.0988087553,2.7571917711 \backslash \mathrm{H},-7.0757055798,1.48$ $67975855,3.6182625903 \backslash \mathrm{H},-5.7631132575,1.2849464444,2.4856705314 \backslash \backslash$ Versi on $=E M 64 \mathrm{~L}-\mathrm{G} 0$ 9RevA. 02 $\backslash$ Sta te $=1-\mathrm{A} \backslash \mathrm{HF}=-1958.5665228 \backslash \mathrm{RMSD}=3.109 \mathrm{e}-09 \backslash \mathrm{RMSF}=6.3$ $45 e-06 \backslash$ Dipole=0.1614048,0.5253991,0.793955 Quadrupole=201.0279481, -97 . $5682165,-103.4597316,0.7428521,-30.9163168,13.971673 \backslash \mathrm{PG}=\mathrm{C} 01 \quad[\mathrm{X}(\mathrm{C} 26 \mathrm{H} 41 \mathrm{~N}$ $906)] \backslash \backslash 0$

Table S28. M06-2X/6-31+G(d,p) Optimized Geometry of AAWAR dication F. opt $\mathrm{m062x} / 6-31+\mathrm{G}(\mathrm{d}, \mathrm{p}) \quad \mathrm{scf}=(\mathrm{tight}, \mathrm{xqc})$ cachesize $=13107$

2 geom=allcheck $\backslash \backslash 1-811 \backslash \backslash 2,1 \backslash \mathrm{N},-6.6608412933,-0.0934943774,-0$

$.346067948 \backslash \mathrm{H},-6.0572332388,-0.2626206842,-1.1724941199 \backslash \mathrm{H},-7.6409737686$ $,-0.2577478233,-0.583429417 \backslash \mathrm{H},-6.3870873669,-0.7995894965,0.3646949875$ $\backslash \mathrm{C},-6.3967350893,1.3024715971,0.1296621584 \backslash \mathrm{H},-7.110286791,1.9565307961$ $,-0.3823483536 \backslash \mathrm{C},-6.5573662027,1.3927164969,1.6414692966 \backslash \mathrm{H},-6.50844688$ $46,2.4317182591,1.9744578076 \backslash \mathrm{H},-5.7770340877,0.8202086468,2.1513617397$ $\backslash \mathrm{H},-7.5351202076,1.0123028559,1.9486731702 \backslash \mathrm{C},-4.9930736137,1.630280290$ $2,-0.4121304059 \backslash 0,-4.5250338562,0.9185934908,-1.302018416 \backslash \mathrm{N},-4.3677191$ $927,2.6924944978,0.1028104245 \backslash \mathrm{H},-4.7897686643,3.2095525533,0.863414004$ $2 \backslash \mathrm{C},-3.075060991,3.1761008317,-0.4031303378 \backslash \mathrm{H},-3.059303501,2.945696010$ $8,-1.4748595976 \backslash \mathrm{C},-2.9563721545,4.6724018171,-0.1693608943 \backslash \mathrm{H},-3.766847$ $1085,5.1994894924,-0.6769141329 \backslash \mathrm{H},-2.0030415994,5.0327291405,-0.556122$ $8443 \backslash \mathrm{H},-2.976151233,4.9023891748,0.8997100144 \backslash \mathrm{C},-1.9082237661,2.410964$ $1407,0.2552763714 \backslash 0,-0.9904578874,2.9989605017,0.8146738716 \backslash \mathrm{N},-1.97934$ 
$41201,1.0640582134,0.1624179025 \backslash \mathrm{H},-2.668860054,0.6342213701,-0.4497251$ $842 \backslash \mathrm{C},-0.9961364232,0.1882866632,0.7829184075 \backslash \mathrm{H},-0.652640699,0.6888812$ $381,1.6951206626 \backslash \mathrm{C},-1.6314705262,-1.1616266572,1.1499864823 \backslash \mathrm{H},-0.82470$ $78795,-1.8765543644,1.3375338041 \backslash \mathrm{H},-2.161779002,-1.0241189902,2.097121$ $2922 \backslash \mathrm{C},-2.6010704578,-1.7234469711,0.1443399345 \backslash \mathrm{C},-2.3752833195,-2.054$ $0406531,-1.1697645329 \backslash \mathrm{H},-1.4629361625,-1.964582518,-1.7424347393 \backslash \mathrm{N},-3$. $5140506485,-2.5902475608,-1.7238118521 \backslash \mathrm{H},-3.5775881639,-2.9583478157$,$2.6611480949 \backslash \mathrm{C},-4.4998832922,-2.6553314368,-0.7748092479 \backslash \mathrm{C},-3.96500848$ $77,-2.098110574,0.4197952015 \backslash \mathrm{C},-4.7711806841,-2.0824852835,1.574492805$ $5 \backslash \mathrm{H},-4.387466087,-1.6957153689,2.5150560203 \backslash \mathrm{C},-6.0510757065,-2.6275430$ $708,1.5188440875 \backslash \mathrm{H},-6.6598207221,-2.6628445431,2.4169624124 \backslash \mathrm{C},-5.79096$ $06299,-3.1974104079,-0.8409097569 \backslash \mathrm{H},-6.1665984131,-3.6516952244,-1.752$ $9928891 \backslash \mathrm{C},-6.5543801849,-3.1850372687,0.3199431473 \backslash \mathrm{H},-7.5415985062,-3$. $6361584857,0.3159212458 \backslash \mathrm{C}, 0.2179446545,0.0320190544,-0.1457003448 \backslash 0,0$. $4255899279,-0.9917291272,-0.7924837806 \backslash N, 1.0307613356,1.1118209705,-0$. $2016218032 \backslash \mathrm{H}, 0.7470354181,1.9469450203,0.3075253141 \backslash \mathrm{C}, 2.0783597103,1.2$ $353440354,-1.2115983129 \backslash \mathrm{H}, 1.7277892252,0.7087631622,-2.1083785658 \backslash \mathrm{C}, 2$. $3319794737,2.7023837686,-1.5178257263 \backslash \mathrm{H}, 3.097941058,2.8035793547,-2.28$ $8015715 \backslash \mathrm{H}, 2.6745961231,3.2307495683,-0.6239871196 \backslash \mathrm{H}, 1.4114285419,3.167$ $9567265,-1.8768467301 \backslash \mathrm{C}, 3.3660197366,0.5276325518,-0.7596614466 \backslash 0,4.42$ $34448298,1.1537764925,-0.5740834803 \backslash C, 4.1681110285,-3.0184534433,-0.68$ $96661442 \backslash 0,3.0990741873,-3.4028885033,-1.085865942 \backslash 0,5.2391643883,-3.7$ $998572985,-0.5174698021 \backslash \mathrm{H}, 5.0081606348,-4.7080020947,-0.7746035717 \backslash \mathrm{N}, 3$ $.2876692714,-0.7969985339,-0.5990034342 \backslash \mathrm{H}, 2.4140777251,-1.2830091616,-$ $0.8188245267 \backslash \mathrm{C}, 4.4646373994,-1.5814865816,-0.2868470142 \backslash \mathrm{H}, 5.2950507841$ $,-1.2281366461,-0.9141891171 \backslash \mathrm{C}, 4.8822797805,-1.5089095354,1.2062915961$ $\backslash \mathrm{H}, 4.5630932643,-0.5338077524,1.5938213651 \backslash \mathrm{H}, 4.3107962173,-2.254370288$ $1,1.7668549532 \backslash \mathrm{C}, 6.3964582533,-1.7132858467,1.4246057346 \backslash \mathrm{H}, 6.840216949$ $9,-2.1946786741,0.5478528923 \backslash \mathrm{H}, 6.5701717497,-2.3912266824,2.2635659828$ $\backslash \mathrm{C}, 7.1496187315,-0.4169433918,1.7271433943 \backslash \mathrm{H}, 6.8804945691,-0.038953009$ $6,2.7221991047 \backslash \mathrm{H}, 8.2243821556,-0.6267266174,1.713713015 \backslash \mathrm{N}, 6.8323908043$ $, 0.5967730125,0.7207192353 \backslash \mathrm{H}, 5.9203710126,0.5493035575,0.2526065562 \backslash \mathrm{C}$, $7.4344356026,1.7731918458,0.5971416439 \backslash N, 8.5819022025,2.0345667866,1.2$ $345263896 \backslash \mathrm{H}, 9.0314904081,2.9337845217,1.1563064106 \backslash \mathrm{H}, 9.054378294,1.324$ $6860275,1.7714218907 \backslash \mathrm{N}, 6.8725252437,2.7051303914,-0.1800732821 \backslash \mathrm{H}, 7.349$ $5004244,3.5638056002,-0.4047895542 \backslash \mathrm{H}, 5.9576975513,2.525312237,-0.58394$ $45524 \backslash \backslash$ Version=EM6 4L-G0 9RevA. 02 \State $=1-A \backslash H F=-1958.5653823 \backslash$ RMSD $=2.535 e$ $-09 \backslash \mathrm{RMSF}=4.120 \mathrm{e}-06 \backslash \mathrm{Dipole}=0.0140135,2.5564189,0.8147724 \backslash \mathrm{Quadrupole}=196$ $.4174254,-82.4394122,-113.9780132,17.4412421,19.5518634,2.8600736 \backslash P G=C$ $01[\mathrm{X}(\mathrm{C} 26 \mathrm{H} 41 \mathrm{~N} 906)] \backslash \backslash \mathrm{Q}$ 